\title{
Direct Synthesis of Monofunctionalized Indolizine Derivatives Bearing Alkoxymethyl Substituents at C-3 and their Benzofused Analogs
}

\author{
Joseph Kaloko, Jr. and Anthony Hayford* \\ Department of Chemistry, Thomas Harriot College of Arts and Sciences, East Carolina \\ University, Science and Technology Building, Suite 300 Greenville, NC 27857-4343 \\ hayforda@mail.ecu.edu
}

Table of Contents

Pages

1. Detailed experimental procedures 2-18

2. References for known compounds 19

3. ${ }^{1} \mathrm{H}$ and ${ }^{13} \mathrm{C}$ NMR spectra data 20-64 


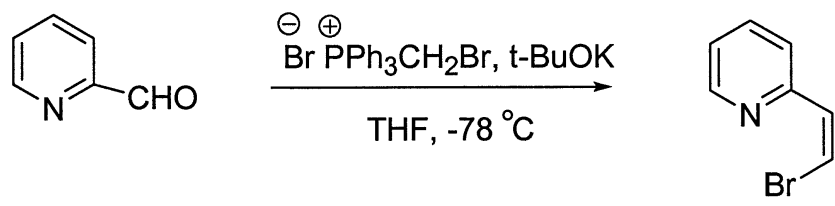

(Z)-2-( $\beta$-bromovinyl) pyridine: To a cooled $\left(-78^{\circ} \mathrm{C}\right)$ suspension of bromomethyltriphenylphosphonium bromide $(25.27 \mathrm{~g}, 50.0 \mathrm{mmol})$ in dried THF (150 $\mathrm{mL})$ under a nitrogen atmosphere, was added potassium tert-butoxide $(6.57 \mathrm{~g}, 50.0$ mmol). The resulting yellow mixture was stirred at the indicated temperature for $1 \mathrm{hr}$. A solution of 2-pyridine carboxaldehyde $(5 \mathrm{~mL}, 42.0 \mathrm{mmol})$ in dried THF $(10 \mathrm{~mL})$ was then introduced via a syringe. The temperature was maintained at $-78{ }^{\circ} \mathrm{C}$, and the mixture was stirred an additional $5 \mathrm{hrs}$. The mixture was diluted with $80 \mathrm{~mL}$ of petroleum ether, and filtered under vacuum. Evaporation of the solvent and purification by flash column chromatography (silica gel, $30 \%$ ethyl acetate in petroleum ether) gave vinyl bromide $(7.29 \mathrm{~g}, 95 \%)$ as a yellow oil. The product contains $\mathrm{Z}$ and $\mathrm{E}$ isomers in 9:1 $\mathrm{Z} / \mathrm{E}$ ratio. Spectroscopic data obtained for the Z-isomer were in agreement with that reported. ${ }^{1}{ }^{1} \mathrm{H}$ NMR (500 MHz, $\left.\mathrm{CDCl}_{3}\right) \delta 6.66(1 \mathrm{H}, \mathrm{d}, J=8.5 \mathrm{~Hz}), 7.23(1 \mathrm{H}$, ddd, $J=8.0,5.0,1.5 \mathrm{~Hz})$, $7.26(1 \mathrm{H}, \mathrm{d}, J=8.5 \mathrm{~Hz}), 7.69(1 \mathrm{H}, \mathrm{td}, J=8.0,1.5 \mathrm{~Hz}), 8.01(1 \mathrm{H}, \mathrm{td}, J=8.0,1.5 \mathrm{~Hz}), 8.64$ $(1 \mathrm{H}, \mathrm{td}, J=5.0,1.5 \mathrm{~Hz}) ;{ }^{13} \mathrm{C} \mathrm{NMR}\left(75 \mathrm{MHz}, \mathrm{CDCl}_{3}\right) \delta 109.4,122.9,123.9,133.4$, 136.1, 149.7, 154.0.

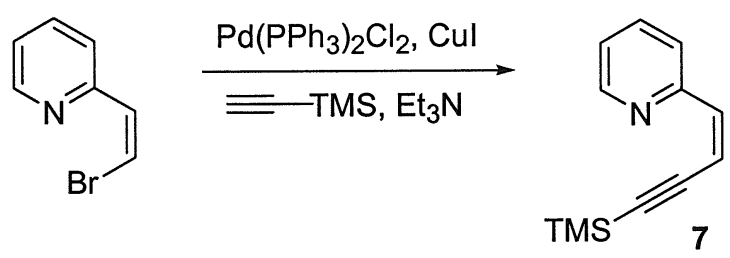

(Z)-2-(4-Trimethylsilylbut-1-en-3-ynyl) pyridine 7: To a solution of monobromide $(2.60 \mathrm{~g}, 14.1 \mathrm{mmol})$ in triethylamine $(50 \mathrm{~mL})$ was added $\mathrm{Pd}\left[\mathrm{PPh}_{3}\right]_{2} \mathrm{Cl}_{2}(0.198 \mathrm{~g}, 0.028$ 
mmol). After stirring for $10 \mathrm{~min}$, copper iodide $(0.134 \mathrm{~g}, 0.071 \mathrm{mmol})$ and trimethylsilylacetylene $(2.40 \mathrm{~mL}, 16.9 \mathrm{mmol})$ were added to the mixture. The resulting mixture was further stirred for $6 \mathrm{hrs}$ at room temperature. After evaporation of the solvent under reduced pressure, the residue was dissolved in hexanes $(40 \mathrm{~mL})$ and filtered through Celite. The hexanes solution was washed with water, dried over sodium sulfate, and the solvent removed under reduced pressure. The residue was purified by flash column chromatography on silica gel using petroleum ether/ ethyl acetate $(15: 1 \rightarrow 10: 1)$ as eluent to afford the titled compound. ${ }^{2}$ Yield; $2.83 \mathrm{~g}(100 \%)$ as a yellow oil. ${ }^{1} \mathrm{H}$ NMR $\left(300 \mathrm{MHz}, \mathrm{CDCl}_{3}\right) \delta 0.25(9 \mathrm{H}, \mathrm{s}), \delta 5.96(1 \mathrm{H}, \mathrm{d}, J=12.3 \mathrm{~Hz}), 6.88(1 \mathrm{H}, \mathrm{d}, J=12.3 \mathrm{~Hz})$ $7.24(1 \mathrm{H}$, ddd, $J=6.6,4.8,1.2 \mathrm{~Hz}), 7.69(1 \mathrm{H}, \mathrm{td}, J=6.6,1.2 \mathrm{~Hz}), 8.46(1 \mathrm{H}, \mathrm{d}, J=8.1$ $\mathrm{Hz}), 8.60(1 \mathrm{H}, \mathrm{d}, J=4.8 \mathrm{~Hz}) ;{ }^{13} \mathrm{C} \mathrm{NMR}\left(125 \mathrm{MHz}, \mathrm{CDCl}_{3}\right) \delta$ 0.0, 103.3, 104.2, 111, 123.2, 136, 140.9, 149.6, 155.3; IR (neat $\mathrm{cm}^{-1}$ ) 3057, 2966, 2341, 2141, 2067, 1584, 1392, 1250, 1153, 1050, 1020, 986, 836; MS (EI) m/z (rel. intensity) $201\left(\mathrm{M}^{+}, 0\right), 200$ (base), 186 (60), 170 (17), 156 (20), 141 (10), 132 (35), 130 (6), 106 (6), 83 (5), 78 (5), 67 (5), 53 (5); HRMS calculated for $\mathrm{C}_{12} \mathrm{H}_{15} \mathrm{NSi}$ 201.0974, found 201.0935.
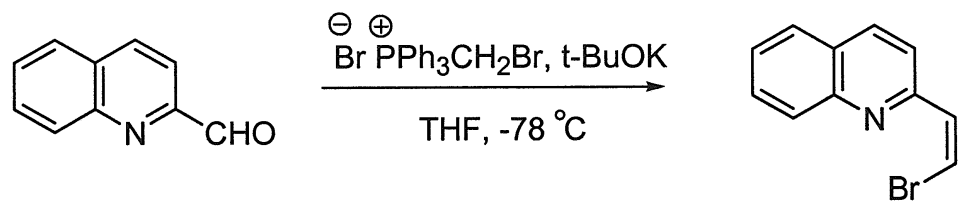

Synthesis of $(\mathbb{Z})-2-(\beta$-bromovinyl) quinoline: This compound was synthesized analogously to the procedure above, from bromomethyltriphenylphosphonim bromide (20.81 g, $47.72 \mathrm{mmol})$, potassium tert-butoxide (5.36 g, $47.72 \mathrm{mmol})$ and 2-quinoline carboxaldehyde $(6.00 \mathrm{~g}, 38.18 \mathrm{mmol})$ in $200 \mathrm{~mL}$ of anhydrous THF. Purification by flash column chromatography on silica gel (10:1 petroleum ether/ ethyl acetate) gave 
$6.59 \mathrm{~g}(80 \%)$ product as a yellow oil. The product contains $\mathrm{Z}$ and $\mathrm{E}$ isomers in 13:1 Z/E ratio. ${ }^{1} \mathrm{H}$ NMR (cis isomer) ${ }^{1}\left(300 \mathrm{MHz}, \mathrm{CDCl}_{3}\right) \delta 6.79(1 \mathrm{H}, \mathrm{d}, J=8.1 \mathrm{~Hz}), 7.45(1 \mathrm{H}, \mathrm{d}$, $J=8.1 \mathrm{~Hz}), 7.55(1 \mathrm{H}, \mathrm{td}, J=6.9,1.2 \mathrm{~Hz}), 7.72(1 \mathrm{H}, \mathrm{td}, J=6.9,1.2 \mathrm{~Hz}), 7.81(1 \mathrm{H}, \mathrm{dd}, J=$ 8.1, $1.2 \mathrm{~Hz}), 8.07,(1 \mathrm{H}, \mathrm{d}, J=8.6 \mathrm{~Hz}), 8.11,(1 \mathrm{H}, \mathrm{d}, J=8.6 \mathrm{~Hz}), 8.17,(1 \mathrm{H}, \mathrm{d}, J=8.6 \mathrm{~Hz})$; ${ }^{13} \mathrm{C} \mathrm{NMR}\left(75 \mathrm{MHz}, \mathrm{CDCl}_{3}\right) \delta 110.70,121.50,127.14,127.54,127.78,129.61,130.01$, $134.05,136.14,148.23,154.59$.<smiles>Br/C=C\c1ccc2ccccc2n1</smiles>
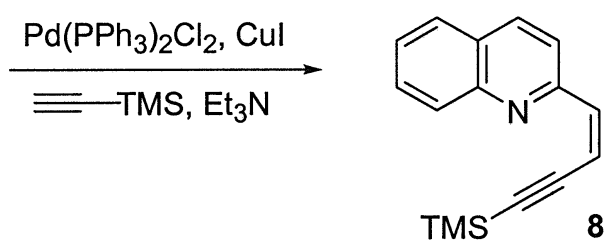

(Z)-2-(4-Trimethylsilylbut-1-en-3-ynyl) quinoline 8: Compound 8 was synthesized analogously to 7 from vinyl bromide $(5.92 \mathrm{~g}, 25.30 \mathrm{mmol})$, trimethylsilylacetylene (4.30 $\mathrm{mL}, 30.36 \mathrm{mmol}), \mathrm{CuI}(0.24 \mathrm{~g}, 1.27 \mathrm{mmol})$, and $\mathrm{Pd}\left[\mathrm{PPh}_{3}\right]_{2} \mathrm{Cl}_{2}(0.44 \mathrm{~g}, 0.06 \mathrm{mmol})$ in 100 $\mathrm{mL}$ of triethylamine. The residue was purified by flash column chromatography on silica gel using 15:1 hexanes/ ethyl acetate as eluent. The protected quinoline-enyne was obtained as a yellow solid (4.83 $\mathrm{g}, 76 \%), \mathrm{mp}=49-51^{\circ} \mathrm{C} . \quad{ }^{1} \mathrm{H} \mathrm{NMR}\left(300 \mathrm{MHz}, \mathrm{CDCl}_{3}\right)$ $\delta$ 0.25, (9H, s), $6.10(1 \mathrm{H}, \mathrm{d}, J=12.3 \mathrm{~Hz}), 7.06(1 \mathrm{H}, \mathrm{d}, J=12.3 \mathrm{~Hz}), 7.52(1 \mathrm{H}, \mathrm{td}, J=6.9$, $1.2 \mathrm{~Hz}), 7.70(1 \mathrm{H}, \mathrm{td}, J=6.9,1.2 \mathrm{~Hz}), 7.78(1 \mathrm{H}, \mathrm{d}, J=8.7), 8.05(1 \mathrm{H}, \mathrm{d}, J=8.7), 8.13$ $(1 \mathrm{H}, \mathrm{d}, J=8.7), 8.58(1 \mathrm{H}, \mathrm{d}, J=8.7) ;{ }^{13} \mathrm{C} \mathrm{NMR}\left(75 \mathrm{MHz}, \mathrm{CDCl}_{3}\right) \delta 0.00,103.16$, $104.51,112.59,120.93,127.04,127.66,127.75,129.73,129.87,135.96,141.21,148.22$, 155.59; MS (EI) m/z (rel. intensity) $251\left(\mathrm{M}^{+}, 48\right), 236$ (35), 220 (14), 206 (20), 191 (24), 178 (base), 156 (11), 128 (10), 110 (9), 101 (4), 75 (5), 73 (89), 53 (5) 43 (4); HRMS calculated for $\mathrm{C}_{16} \mathrm{H}_{17} \mathrm{NSi} 251.1130$, found 251.1140. 

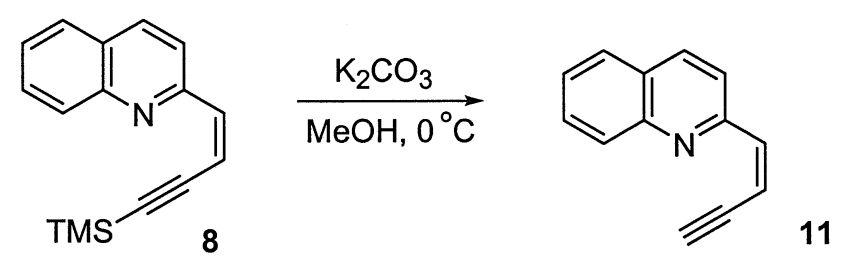

(Z) -2-(But-1-en-3-ynyl) quinoline 11: A solution of silylated enyne 8 (0.20 g, 0.8 mmol $)$ in $40 \mathrm{~mL}$ of methanol was cooled to $0{ }^{\circ} \mathrm{C}$ and then potassium carbonate $(0.21 \mathrm{~g}$, $1.6 \mathrm{mmol}$ ) was added to the mixture. The mixture was stirred for $1 \mathrm{hr}$ at the indicated temperature, diluted with a saturated solution of sodium bicarbonate and extracted with cold hexanes. The organic layer was dried over sodium sulfate and concentrated on a rotary evaporator. The crude was purified by flash column chromatography on neutral alumina using 5:1 hexanes/ethyl acetate as the eluent to yield the product as a brown solid (0.13 g, 94\%). ${ }^{1} \mathrm{H}$ NMR (300 MHz, $\left.\mathrm{CDCl}_{3}\right) \delta 3.47(1 \mathrm{H}, \mathrm{dd}, J=0.9,2.7 \mathrm{~Hz}), 6.08$ $(1 \mathrm{H}, \mathrm{dd}, J=12.3,2.7 \mathrm{~Hz}), 7.12(1 \mathrm{H}, \mathrm{d}, J=12.3 \mathrm{~Hz}), 7.54(1 \mathrm{H}, \mathrm{td}, J=6.9,1.2 \mathrm{~Hz}), 7.68$ $(1 \mathrm{H}, \mathrm{td}, J=6.9,1.2 \mathrm{~Hz}), 7.80(1 \mathrm{H}, \mathrm{d}, J=8.4 \mathrm{~Hz}), 8.06(1 \mathrm{H}, \mathrm{d}, J=8.4 \mathrm{~Hz}), 8.16(1 \mathrm{H}, \mathrm{d}, J$ $=8.4 \mathrm{~Hz}), 8.51(1 \mathrm{H}, \mathrm{d}, J=8.4 \mathrm{~Hz}) ;{ }^{13} \mathrm{C} \mathrm{NMR}\left(75 \mathrm{MHz}, \mathrm{CDCl}_{3}\right) \delta 81.50,86.06,111.48$, $120.72,127.12,127.68,127.76,129.69,129.93,136.30,142.01,148.17,155.23$.

\section{Synthesis of 3-alkoxymethylindolizine and 1-alkoxymethylpyrrolo [1,2-a] quinoline}

\section{General Procedure A: Using alcohols with "low" boiling points ${ }^{3}$}

To a solution of silylated enyne 7 or $8(1 \mathrm{mmol})$ in the appropriate alcohol $(20 \mathrm{~mL})$ was added $\mathrm{KF}$ (2.0 mmol) or CsF (1.5 mmol). The mixture was heated and kept under reflux until 7 or 8 was no longer detectable (TLC analysis). The solvent ("low" boiling alcohol) was removed in vacuo. The residue was dissolved in petroleum ether or hexanes and 
washed with water $(3 \times 30 \mathrm{~mL})$. The organic layer was dried over sodium sulfate or magnesium sulfate under nitrogen and concentrated to yield the pure products.

\section{General Procedure B: Using alcohols with "high" boiling points ${ }^{3}$}

The synthetic procedure is similar to General Procedure A. However, the work-up procedure differs. In this case, the reaction mixture was poured into a solution of $\mathrm{MeOH} / \mathrm{H}_{2} \mathrm{O}(1: 1) 40 \mathrm{~mL}$ and extracted with petroleum ether $(3 \times 20 \mathrm{~mL})$. The organic layer was dried over sodium sulfate or magnesium sulfate under nitrogen and concentrated. The crude product was flash chromatographed on silica gel (benzoindolizine derivatives) or basic alumina (indolizine derivatives) using hexanes/ethyl acetate as eluent.

\section{General Procedure C: Expensive and/or "high" boiling alcohols ${ }^{3}$} A suspension of silylated enyne 7 or $8(1 \mathrm{mmol}), \mathrm{CsF}(1.5 \mathrm{mmol})$ and $10 \mathrm{mmols}$ of the appropriate alcohol in anhydrous toluene $(20 \mathrm{~mL})$ was well-stirred at reflux until the disappearance of the starting material 7 or $\mathbf{8}$. The solvent was evaporated in vacuo, and the residue purified by flash chromatography $\left(\mathrm{SiO}_{2}\right.$ or basic alumina, petroleum ether/ ethyl acetate).
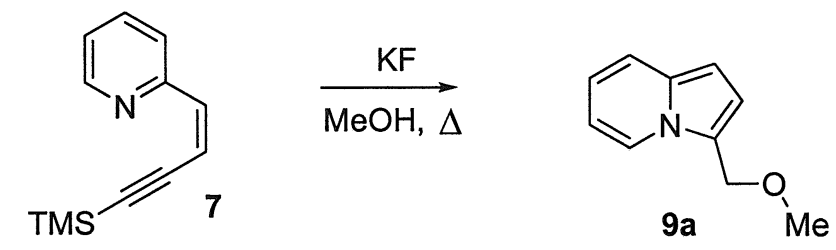

3-Methoxymethylindolizine 9a: Synthesized according to general procedure A from KF $(0.058 \mathrm{~g}, 0.99 \mathrm{mmol})$ and silylated enyne $7(0.100 \mathrm{~g}, 0.49 \mathrm{mmol})$ in $20 \mathrm{~mL}$ of methanol for $1 \mathrm{hr}$. Yield; (0.072 $\mathrm{g}, 90 \%)$ as yellow oil. Due to the unstable nature of this 
compound, it should not be exposed to air for long periods and should be stored under nitrogen. ${ }^{1} \mathrm{H}$ NMR $\left(300 \mathrm{MHz}, \mathrm{CDCl}_{3}\right) \delta 3.30(3 \mathrm{H}, \mathrm{s}), \delta 4.74(2 \mathrm{H}, \mathrm{s}), \delta 6.43(1 \mathrm{H}, \mathrm{d}, J=$ $3.9 \mathrm{~Hz}), \delta 6.56(1 \mathrm{H}, \mathrm{td}, J=7.5,1.2 \mathrm{~Hz}), \delta 6.77(2 \mathrm{H}, \mathrm{m}), \delta 7.41(1 \mathrm{H}, \mathrm{d}, J=7.5 \mathrm{~Hz}), \delta 8.04$ $(1 \mathrm{H}, \mathrm{dd}, J=7.5,1.2 \mathrm{~Hz}) ;{ }^{13} \mathrm{C} \mathrm{NMR}\left(75 \mathrm{MHz}, \mathrm{CDCl}_{3}\right) \delta 57.2,66.1,98.3,110.6,115.7$, $117.5,119.9,122.1,123.5,134.3$.
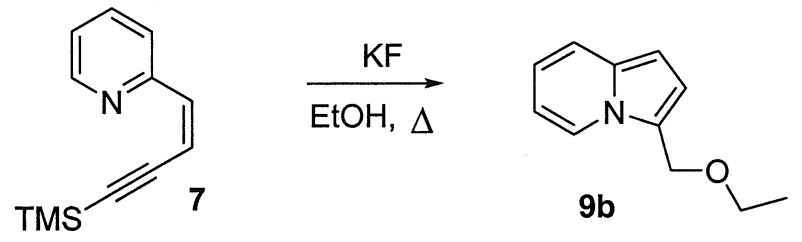

3-Ethoxymethylindolizine 9b: Compound $9 \mathrm{~b}$ was synthesized according to general procedure A from compound 7 (0.100 g, $0.49 \mathrm{mmol})$ and $\mathrm{KF}(0.058 \mathrm{~g}, 0.99 \mathrm{mmol})$ in 20 $\mathrm{mL}$ of ethanol for $1 \mathrm{hr}$. Yield; (0.080 g, 92\%) as a yellow oil. ${ }^{1} \mathrm{H}$ NMR $(300 \mathrm{MHz}$, $\left.\mathrm{CDCl}_{3}\right) \delta 1.21(3 \mathrm{H}, \mathrm{t}, J=6.9 \mathrm{~Hz}), \delta 3.50(2 \mathrm{H}, \mathrm{q}, J=6.9 \mathrm{~Hz}), \delta 4.77(2 \mathrm{H}, \mathrm{s}), \delta 6.38(1 \mathrm{H}$, $\mathrm{d}, J=3.9 \mathrm{~Hz}), \delta 6.55(1 \mathrm{H}, \mathrm{td}, J=6.9,1.2 \mathrm{~Hz}), \delta 6.75(2 \mathrm{H}, \mathrm{m}), \delta 7.39(1 \mathrm{H}, \mathrm{td}, J=6.9$, $1.2 \mathrm{~Hz}), \delta 8.05(1 \mathrm{H}, \mathrm{dd}, J=6.9,1.2 \mathrm{~Hz}) ;{ }^{13} \mathrm{C} \mathrm{NMR}\left(75 \mathrm{MHz}, \mathrm{CDCl}_{3}\right) \delta 15.4,64.3,64.9$, 98.2, 110.5, 115.3, 117.3, 119.5, 120.4, 123.5, 134.2; IR (neat, $\mathrm{cm}^{-1}$ ) 2975, 2870, 2340, $1630,1503,1360,1315,1245,1158,1087,753 ; \mathrm{MS}(\mathrm{EI}) \mathrm{m} / \mathrm{z}$ (rel. intensity) $175(\mathrm{M}+$, 20), 130 (base), 117 (5), 103 (3), 90 (3), 78 (5) 51 (3); HRMS calculated for $\mathrm{C}_{11} \mathrm{H}_{13} \mathrm{NO}$ 175.0997, found 175.0993. 

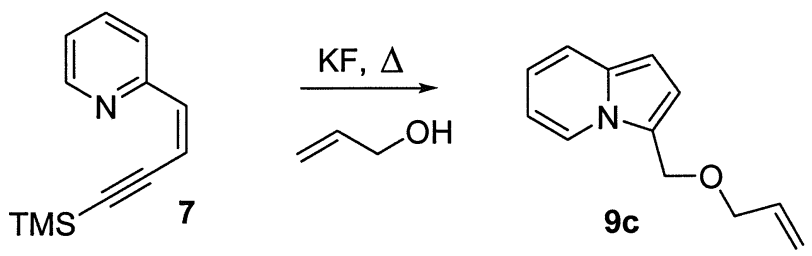

3-Allyloxymethylindolizine 9c: Compound 9c was synthesized according to general procedure A from compound $7(0.100 \mathrm{~g}, 0.49 \mathrm{mmol})$ and $\mathrm{KF}(0.058 \mathrm{~g}, 0.99 \mathrm{mmol})$ in 20 $\mathrm{mL}$ of allyl alcohol for $45 \mathrm{~min}$. Yield; $(0.093 \mathrm{~g}, 100 \%)$ as a yellow oil. ${ }^{1} \mathrm{H}$ NMR (300 $\left.\mathrm{MHz}, \mathrm{CDCl}_{3}\right) \delta 3.98(2 \mathrm{H}, \mathrm{td}, J=2.7,1.5 \mathrm{~Hz}), \delta 4.82(2 \mathrm{H}, \mathrm{s}), \delta 5.34(2 \mathrm{H}, \mathrm{m}), \delta 5.98,(1 \mathrm{H}$, $\mathrm{m}), \delta 6.41(1 \mathrm{H}, \mathrm{d}, J=3.9 \mathrm{~Hz}), \delta 6.58(1 \mathrm{H}, \mathrm{td}, J=7.0,1.2 \mathrm{~Hz}), \delta 6.79(2 \mathrm{H}, \mathrm{m}), \delta 7.42$ $(1 \mathrm{H}, \mathrm{d}, J=7.0 \mathrm{~Hz}), \delta 8.09(1 \mathrm{H}, \mathrm{dd}, J=7.0,1.2 \mathrm{~Hz}) ;{ }^{13} \mathrm{C}\left(75 \mathrm{MHz}, \mathrm{CDCl}_{3}\right) \delta 63.6,70.2$, 98.3, 110.6, 115.6, 117.5, 117.7, 119.4, 119.9, 123.5, 134.3, 134.9; IR (neat, $\mathrm{cm}^{-1}$ ) 3079, 2901, 2340, 1631, 1537, 1502, 1360, 1204, 1054, 926, 818; HRMS calculated for $\mathrm{C}_{12} \mathrm{H}_{11} \mathrm{NO} 187.0997$ found 187.1006.
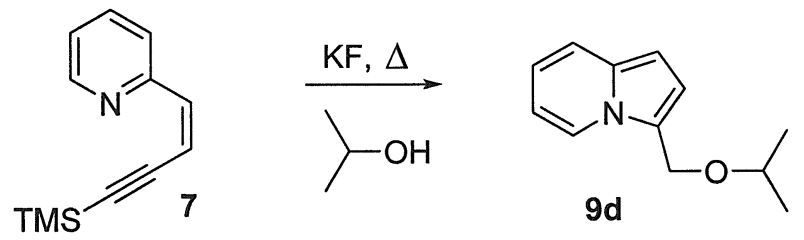

3-Isopropoxymethylindolizine 9d: Compound 9d was synthesized analogously to general procedure A from compound $7(0.100 \mathrm{~g}, 0.49 \mathrm{mmol})$ and $\mathrm{KF}(0.058 \mathrm{~g}, 0.99$ $\mathrm{mmol})$ in $20 \mathrm{~mL}$ of 2-propanol for $1.5 \mathrm{hr}$. Yield; $(0.089 \mathrm{~g}, 94 \%)$ as a yellow oil. ${ }^{1} \mathrm{H}$ NMR (300 MHz, $\left.\mathrm{CDCl}_{3}\right) \delta 1.21(6 \mathrm{H}, \mathrm{d}, J=2.4 \mathrm{~Hz}), \delta 3.67(1 \mathrm{H}, \mathrm{m}), \delta 4.80(2 \mathrm{H}, \mathrm{s}), \delta$ $6.41(1 \mathrm{H}, \mathrm{d}, J=3.6 \mathrm{~Hz}), \delta 6.55(1 \mathrm{H}, \mathrm{td}, J=7.2,1.2 \mathrm{~Hz}), \delta 6.77(2 \mathrm{H}, \mathrm{m}), \delta 7.41(1 \mathrm{H}, \mathrm{d}, J$ $=7.2 \mathrm{~Hz}), \delta 8.08(1 \mathrm{H}, \mathrm{d}, J=7.2 \mathrm{~Hz}) ;{ }^{13} \mathrm{C} \mathrm{NMR}\left(75 \mathrm{MHz}, \mathrm{CDCl}_{3}\right) \delta 22.3,61.9,69.9$, 98.2, 110.5, 115.1, 117.3, 119.4, 120.63, 123.6, 134.2; IR: (neat, $\mathrm{cm}^{-1}$ ) 3087, 2977, 2340, 
1632, 1504, 1315, 1245, 1118, 1040, 919, 816, 754; MS (EI) m/z (rel. intensity) 189 (M+, 20), 130 (base), 117 (4), 103 (4), 78 (5), 40 (3); HRMS calculated for $\mathrm{C}_{12} \mathrm{H}_{15} \mathrm{NO}$ 189.1148 found 189.1154 .
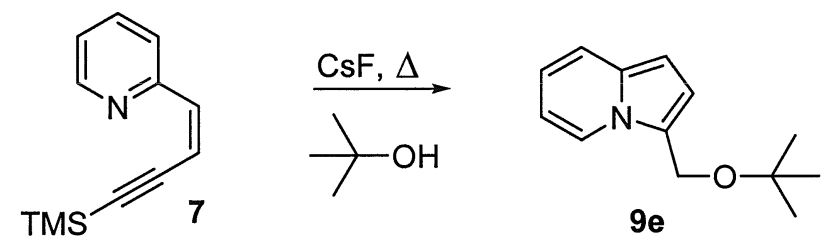

3-tert-Butoxymethylindolizine 9e: Compound 9e was synthesized analogously to general procedure A from compound $7(0.050 \mathrm{~g}, 0.25 \mathrm{mmol})$ and $\mathrm{CsF}(0.057 \mathrm{~g}, 0.37$ $\mathrm{mmol})$ in $20 \mathrm{~mL}$ of tert-butanol for $7 \mathrm{hrs}$. Yield; $(0.046 \mathrm{~g}, 92 \%)$ as a yellow solid, $\mathrm{mp}=$ 53-54 ${ }^{\circ} \mathrm{C} . \quad{ }^{1} \mathrm{H}$ NMR $\left(300 \mathrm{MHz}, \mathrm{CDCl}_{3}\right) \delta 1.34(9 \mathrm{H}, \mathrm{s}), \delta 4.73(2 \mathrm{H}, \mathrm{s}), \delta 6.39(1 \mathrm{H}, \mathrm{d}, J=$ $3.9 \mathrm{~Hz}), \delta 6.53(1 \mathrm{H}, \mathrm{td}, J=7.0,1.2 \mathrm{~Hz}), \delta 6.75(2 \mathrm{H}, \mathrm{m}), \delta 7.39(1 \mathrm{H}, \mathrm{d}, J=7.0 \mathrm{~Hz}), \delta$ $8.04(1 \mathrm{H}, \mathrm{d}, J=7.0 \mathrm{~Hz}) ;{ }^{13} \mathrm{C} \mathrm{NMR}\left(75 \mathrm{MHz}, \mathrm{CDCl}_{3}\right) \delta 27.9,56.5,73.6,98.3,110.3$, 114.5, 117.0, 119.4, 121.3, 123.4, 134.1; IR (in $\left.\mathrm{CHCl}_{3}, \mathrm{~cm}^{-1}\right)$ 2978, 1633, 1364, 1316, 1192, 1047, 909, 740; HRMS calculated for $\mathrm{C}_{13} \mathrm{H}_{17} \mathrm{NO} 203.1310$ found 203.1301 .
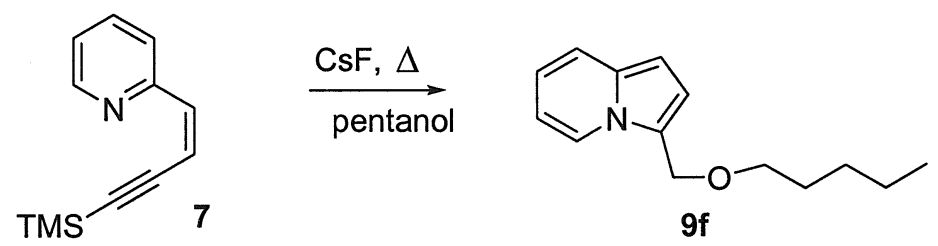

3-Pentyloxymethylindolizine 9f: Synthesized according to general procedure B from protected enyne $7(0.060 \mathrm{~g}, 0.30 \mathrm{mmol})$ and $\mathrm{CsF}(0.068 \mathrm{~g}, 0.48 \mathrm{mmol})$ in $20 \mathrm{~mL}$ of pentanol for $1 \mathrm{hr}$. Yield; $(0.024 \mathrm{~g}, 37 \%)$ as yellow oil. ${ }^{1} \mathrm{H} \mathrm{NMR}\left(500 \mathrm{MHz}, \mathrm{CDCl}_{3}\right) \delta$ $0.86(3 \mathrm{H}, \mathrm{t}, J=7.0 \mathrm{~Hz}), \delta 1.29(4 \mathrm{H}, \mathrm{m}), \delta 1.56(2 \mathrm{H}, \mathrm{m}), \delta 3.39(2 \mathrm{H}, \mathrm{t}, J=6.5 \mathrm{~Hz}), \delta 4.78$ 
$(2 \mathrm{H}, \mathrm{s}), \delta 6.37(1 \mathrm{H}, \mathrm{d}, J=4.0 \mathrm{~Hz}), \delta 6.53(1 \mathrm{H}, \mathrm{td}, J=6.5,1.0 \mathrm{~Hz}), \delta 6.74(2 \mathrm{H}, \mathrm{m}), \delta 7.38$ $(1 \mathrm{H}, \mathrm{td}, J=6.5,1.0 \mathrm{~Hz}), \delta 8.03(1 \mathrm{H}, \mathrm{dd}, J=6.5,1.0 \mathrm{~Hz}) ;{ }^{13} \mathrm{C} \mathrm{NMR}\left(125 \mathrm{MHz}, \mathrm{CDCl}_{3}\right) \delta$ $14.2,22.7,28.6,29.6,64.5,69.6,98.1,110.5,115.3,117.3,119.4,120.4,123.5,134.2$ IR (in $\mathrm{CHCl}_{3}, \mathrm{~cm}^{-1}$ ) 2913, 2848, 2242, 1462, 1316, 1089, 911, 731.
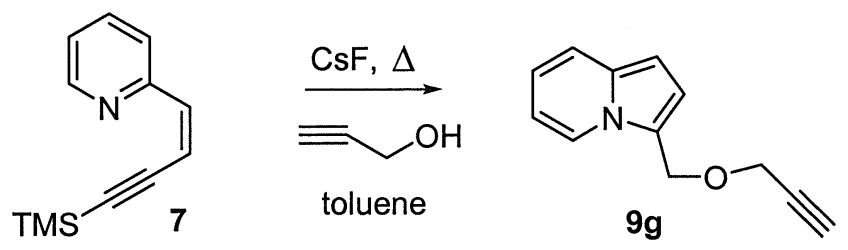

3-Prop-2-ynyloxymethylindolizine 9g: Synthesized according to general procedure C from silylated enyne $7(0.070 \mathrm{~g}, 0.35 \mathrm{mmol}), \mathrm{CsF}(0.079 \mathrm{~g}, 0.52 \mathrm{mmol})$ and propargyl alcohol $(0.195 \mathrm{~g}, 3.5 \mathrm{mmol})$ in $20 \mathrm{~mL}$ of anhydrous toluene for $2 \mathrm{hrs}$. The residue was purified by column chromatography on basic alumina using hexanes and ethyl acetate (20:1) as eluent. Yield; $0.050 \mathrm{~g},(77 \%)$ as a yellow oil. ${ }^{1} \mathrm{H} \mathrm{NMR}\left(500 \mathrm{MHz}, \mathrm{CDCl}_{3}\right) \delta$ $2.47(1 \mathrm{H}, \mathrm{t}, J=2.5 \mathrm{~Hz}), 4.07(2 \mathrm{H}, \mathrm{d}, J=2.5 \mathrm{~Hz}), \delta 4.89(2 \mathrm{H}, \mathrm{s}), \delta 6.39(1 \mathrm{H}, \mathrm{d}, J=3.5$ $\mathrm{Hz}), \delta 6.56(1 \mathrm{H}, \mathrm{td}, J=6.5,1.5 \mathrm{~Hz}), \delta 6.73(1 \mathrm{H}, \mathrm{td}, J=6.5,1.5 \mathrm{~Hz}), \delta 6.82(1 \mathrm{H}, \mathrm{d}, J=$ $3.5 \mathrm{~Hz}), \delta 7.39(1 \mathrm{H}, \mathrm{d}, J=8.0 \mathrm{~Hz}), \delta 8.07(1 \mathrm{H}, \mathrm{d}, J=8.0 \mathrm{~Hz}) ;{ }^{13} \mathrm{C} \mathrm{NMR}(125 \mathrm{MHz}$, $\left.\mathrm{CDCl}_{3}\right) \delta 56.2,62.9,74.9,79.9,98.5,110.7,116.3,117.7,118.9,119.4,123.5,134.5$ HRMS calculated for $\mathrm{C}_{12} \mathrm{H}_{11} \mathrm{NO} 185.0841$ found 185.0841 . 

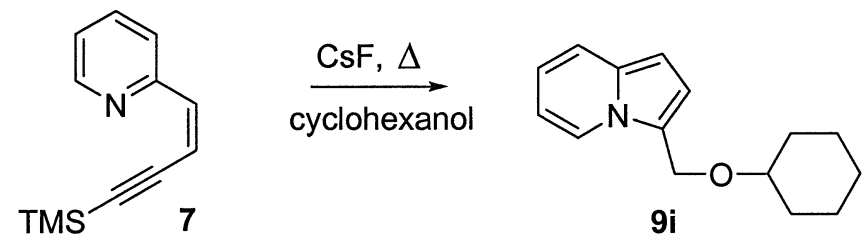

3-Cyclohexyloxymethyindolizine 9i: Compound $9 \mathbf{i}$ was synthesized according to general procedure B from compound $7(0.070 \mathrm{~g}, 0.35 \mathrm{mmol})$ and $\mathrm{CsF}(0.079 \mathrm{~g}, 0.52$ $\mathrm{mmol}$ ) in $20 \mathrm{~mL}$ of cyclohexanol for $1 \mathrm{hr}$. The crude was purified by flash column chromatography (silica gel, 15:1 hexanes/ ethyl acetate and 1.5\% triethylamine) to yield; $0.054 \mathrm{~g},(68 \%)$ as yellow oil. ${ }^{1} \mathrm{H}$ NMR $\left(300 \mathrm{MHz}, \mathrm{CDCl}_{3}\right) \delta 1.33(4 \mathrm{H}, \mathrm{m}), \delta 1.59(2 \mathrm{H}$, $\mathrm{m}), \delta 1.73(2 \mathrm{H}, \mathrm{m}), \delta 1.92(2 \mathrm{H}, \mathrm{m}), \delta 3.34(1 \mathrm{H}, \mathrm{m}), \delta 4.81(2 \mathrm{H}, \mathrm{s}), \delta 6.38(1 \mathrm{H}, \mathrm{d}, J=3.6$ $\mathrm{Hz}), \delta 6.55(1 \mathrm{H}, \mathrm{td}, J=7.2,1.2 \mathrm{~Hz}), \delta 6.74(2 \mathrm{H}, \mathrm{m}), \delta 7.36(1 \mathrm{H}, \mathrm{d}, J=7.2 \mathrm{~Hz}), \delta 8.07$ $(1 \mathrm{H}, \mathrm{d}, J=7.2 \mathrm{~Hz}) ;{ }^{13} \mathrm{C} \mathrm{NMR}\left(75 \mathrm{MHz}, \mathrm{CDCl}_{3}\right) \delta 24.4,26.0,32.4,61.7,76.1,98.2$, $110.4,114.9,117.2,119.3,120.8,123.6,134.2 ;$ IR (neat, $\mathrm{cm}^{-1}$ ) 3087, 2951, 2831, 2658, $2360,1894,1631,1538,1447,1362,1258,1156,1083,949,887,7510 ;$ HRMS calculated for $\mathrm{C}_{15} \mathrm{H}_{19} \mathrm{NO} 229.1467$ found 229.1459 .
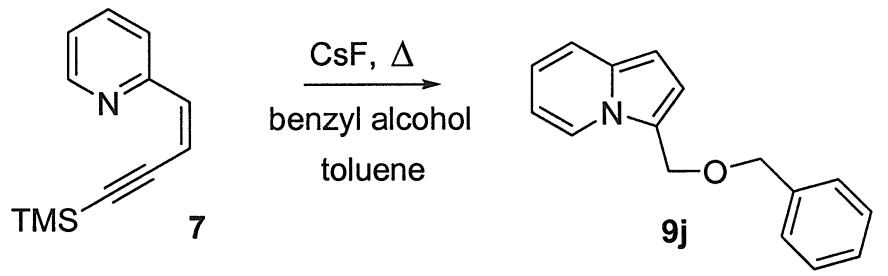

3-Benzyloxymethylindolizine 9j: Compound 9j was synthesized according to general procedure C from compound 7(0.070 g, $0.35 \mathrm{mmol}), \mathrm{CsF}(0.079 \mathrm{~g}, 0.52 \mathrm{mmol})$ and benzyl alcohol $(0.38 \mathrm{~g}, 3.5 \mathrm{mmol})$ in $20 \mathrm{~mL}$ of dry toluene for $2 \mathrm{hrs}$. The crude was purified by flash column chromatography (silica gel, 15:1 hexanes/ ethyl acetate and $1.5 \%$ triethylamine) to yield $0.068 \mathrm{~g},(82 \%)$ as yellow oil. ${ }^{1} \mathrm{H} \mathrm{NMR}\left(300 \mathrm{MHz}, \mathrm{CDCl}_{3}\right) \delta$ 
$4.47(2 \mathrm{H}, \mathrm{s}), \delta 4.85(2 \mathrm{H}, \mathrm{s}), \delta 6.42(1 \mathrm{H}, \mathrm{d}, J=3.9 \mathrm{~Hz}), \delta 6.58(1 \mathrm{H}, \mathrm{td}, J=7.2,1.5 \mathrm{~Hz}), \delta$ $6.74(1 \mathrm{H}, \mathrm{td}, J=7.2,1.5 \mathrm{~Hz}), \delta 6.79(1 \mathrm{H}, \mathrm{d}, J=3.9 \mathrm{~Hz}), \delta 7.43(6 \mathrm{H}, \mathrm{m}), \delta 8.07(1 \mathrm{H}, \mathrm{d} J$ $=7.2 \mathrm{~Hz}) ;{ }^{13} \mathrm{C} \mathrm{NMR}\left(75 \mathrm{MHz}, \mathrm{CDCl}_{3}\right) \delta 63.6,71.2,98.3,110.6,115.8,117.5,119.4$, 119.9, 123.6, 127.2, 127.9, 128.3, 128.7, 128.8, 134.4, 138.3; IR (in $\left.\mathrm{CHCl}_{3}, \mathrm{~cm}^{-1}\right)$ 3030, 2848, 2245, 1462, 1315, 1204, 1086, 911, 730; HRMS calculated for $\mathrm{C}_{16} \mathrm{H}_{15} \mathrm{NO} 237.1154$ found 237.1145 .
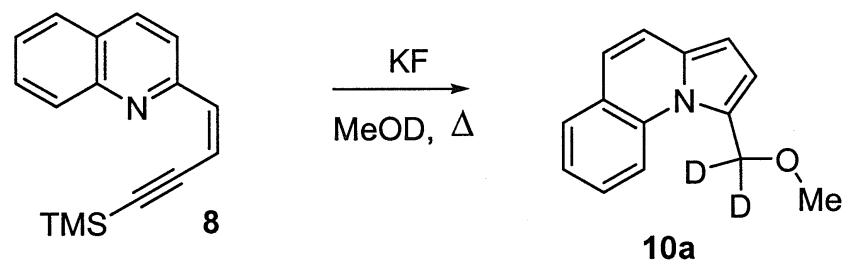

1-Methoxymethylpyrrolo [1,2-a] quinoline 10a: Potassium fluoride $(0.037 \mathrm{~g}, 0.64$ mmol) was added to a solution of the protected enyne $8(0.080 \mathrm{~g}, 0.32 \mathrm{mmol})$ in $20 \mathrm{~mL}$ of single deuterium labeled methanol $\left(\mathrm{CH}_{3} \mathrm{OD}\right)$. The mixture was refluxed for $1 \mathrm{hr}$ and allowed to cool to room temperature. The mixture was diluted with $25 \mathrm{~mL}$ of water and extracted with petroleum ether $(30 \mathrm{~mL} \times 3)$. The petroleum ether layer was dried over sodium sulfate and concentrated to yield the pure product $(0.063 \mathrm{~g}, 87 \%)$ as a yellow oil. ${ }^{1} \mathrm{H}$ NMR $\left(300 \mathrm{MHz}, \mathrm{CDCl}_{3}\right) \delta 3.46(3 \mathrm{H}, \mathrm{s}), \delta 6.51(1 \mathrm{H}, \mathrm{d}, J=3.9 \mathrm{~Hz}), \delta 6.79(1 \mathrm{H}, \mathrm{d}, J=$ $3.9 \mathrm{~Hz}), \delta 7.03(1 \mathrm{H}, \mathrm{d}, J=9.0 \mathrm{~Hz}), \delta 7.37(2 \mathrm{H}, \mathrm{m}), \delta 7.54(1 \mathrm{H}, \mathrm{td} J=7.2,1.5 \mathrm{~Hz}), \delta$ $7.66(1 \mathrm{H}, \mathrm{dd}, J=7.2,1.5 \mathrm{~Hz}), \delta 8.45(1 \mathrm{H}, \mathrm{d}, J=9.0 \mathrm{~Hz}) ;{ }^{13} \mathrm{C} \mathrm{NMR}\left(75 \mathrm{MHz}, \mathrm{CDCl}_{3}\right) \delta$ 56.7, $67.6(\mathrm{~m}), 102.1,117.6,118.0,119.3,119.8,123.8,125.3,125.4,127.9,128.5$, 134.1, 135.2; IR (neat, $\mathrm{cm}^{-1}$ ) 3051, 2986, 2341, 2142, 2080, 1940, 1608, 1552, 1505 , 1383, 1223, 1095, 973, 920, 846, 752. 

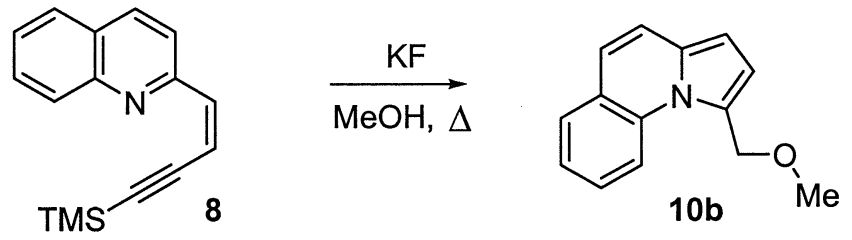

1-Methoxymethylpyrrolo [1,2-a] quinoline 10b: Synthesized according to general procedure A from silylated enyne $8(0.050 \mathrm{~g}, 0.20 \mathrm{mmol})$ and $\mathrm{KF}(0.023 \mathrm{~g}, 0.40 \mathrm{mmol})$ in methanol $(20 \mathrm{~mL})$ for $1 \mathrm{hr}$. Yield; $(0.040 \mathrm{~g}, 95 \%)$ as a yellow oil. ${ }^{1} \mathrm{H}$ NMR $(300 \mathrm{MHz}$, $\left.\mathrm{CDCl}_{3}\right) \delta 3.41(3 \mathrm{H}, \mathrm{s}), \delta 4.87(2 \mathrm{H}, \mathrm{s}), \delta 6.46(1 \mathrm{H}, \mathrm{d}, J=3.7 \mathrm{~Hz}), \delta 6.74(1 \mathrm{H}, \mathrm{d}, J=3.7$ Hz), $\delta 6.98(1 \mathrm{H}, \mathrm{d}, J=9.0 \mathrm{~Hz}), \delta 7.32(2 \mathrm{H}, \mathrm{m}), \delta 7.49(1 \mathrm{H}, \mathrm{td}, J=7.5,1.7 \mathrm{~Hz}), \delta 7.62$ $(1 \mathrm{H}, \mathrm{dd}, J=7.5,1.7 \mathrm{~Hz}), \delta 8.40(1 \mathrm{H}, \mathrm{d}, J=9.0 \mathrm{~Hz}),{ }^{13} \mathrm{C} \mathrm{NMR}\left(75 \mathrm{MHz}, \mathrm{CDCl}_{3}\right) \delta 56.8$, $67.9,102.0,117.6,118.0,119.3,119.8,123.7,125.3,125.4,127.9,128.5,134.1,135.2$ IR (neat, $\mathrm{cm}^{-1}$ ) 3050, 2817, 1694, 1607, 1555, 1479, 1321, 1220, 1123, 1081, 943, 897, 752; HRMS calculated for $\mathrm{C}_{14} \mathrm{H}_{13} \mathrm{NO} 211.0997$ found 211.0997 .
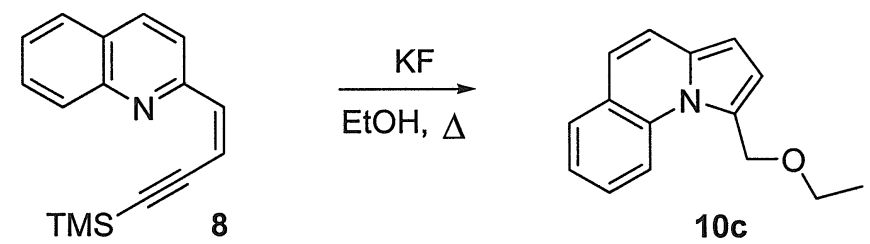

1-Ethoxymethylpyrrolo [1,2-a] quinoline 10c: Compound 10c was synthesized according to general procedure A from compound $8(0.050 \mathrm{~g}, 0.20 \mathrm{mmol})$ and $\mathrm{KF}(0.023$ $\mathrm{g}, 0.40 \mathrm{mmol})$ in $20 \mathrm{~mL}$ of ethanol for $1 \mathrm{hr}$. Yield; $(0.043 \mathrm{~g}, 96 \%)$ as a yellow oil. ${ }^{1} \mathrm{H}$ NMR (300 MHz, $\left.\mathrm{CDCl}_{3}\right) \delta 1.29(3 \mathrm{H}, \mathrm{t}, J=7.2 \mathrm{~Hz}), \delta 3.68(2 \mathrm{H}, \mathrm{q}, J=6.9 \mathrm{~Hz}), \delta 4.94$ $(2 \mathrm{H}, \mathrm{s}), \delta 6.47(1 \mathrm{H}, \mathrm{d}, J=3.9 \mathrm{~Hz}), \delta 6.75(1 \mathrm{H}, \mathrm{d}, J=3.9 \mathrm{~Hz}), \delta 7.01(1 \mathrm{H}, \mathrm{d}, J=8.8 \mathrm{~Hz})$ $\delta 7.34(2 \mathrm{H}, \mathrm{m}), \delta 7.53(1 \mathrm{H}, \mathrm{td}, J=7.6,1.8 \mathrm{~Hz}), \delta 7.64(1 \mathrm{H}, \mathrm{dd}, J=7.6,1.5 \mathrm{~Hz}), \delta 8.48$ $(1 \mathrm{H}, \mathrm{d}, J=8.8 \mathrm{~Hz}) ;{ }^{13} \mathrm{C} \mathrm{NMR}\left(75 \mathrm{MHz}, \mathrm{CDCl}_{3}\right) \delta 15.4,64.7,66.2,102.0,117.7,117.8$, 
$119.3,119.7,123.7,125.3,125.9,127.8,128.5,134.0,135.3 ; \mathbb{R}$ (neat, $\mathrm{cm}^{-1}$ ) 3050, 2976, 2795, 1608, 1555, 1321, 1124, 1081; MS (EI) m/x (rel. intensity) $225\left(\mathrm{M}^{+}, 80\right), 224(2)$, 194 (5), 180 (base), 179 (24), 167 (9), 141 (6), 128 (4), 90 (3); HRMS calculated for $\mathrm{C}_{15} \mathrm{H}_{15} \mathrm{NO} 225.1154$, found 225.1146 .
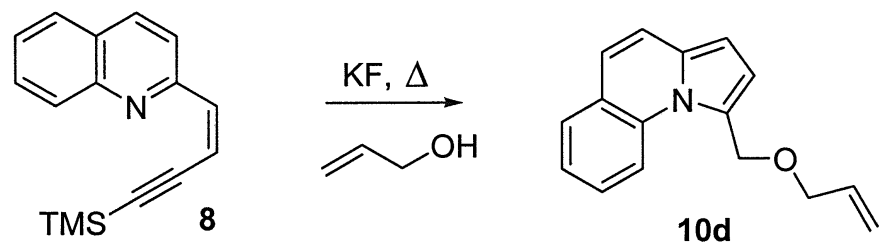

1-Allyloxymethylpyrrolo [1,2-a] quinoline 10d: Compound 10d was synthesized according to general procedure A from compound 8 (0.050 g,0.20 mmol) and KF (0.023 $\mathrm{g}, 0.40 \mathrm{mmol})$ in $20 \mathrm{~mL}$ of allyl alcohol for $45 \mathrm{~min}$. Yield; $(0.047 \mathrm{~g}, 100 \%)$ as a yellow oil. ${ }^{1} \mathrm{H}$ NMR (300 MHz, $\left.\mathrm{CDCl}_{3}\right) \delta 4.15(2 \mathrm{H}, \mathrm{td}, J=2.7,1.2 \mathrm{~Hz}), \delta 4.99(2 \mathrm{H}, \mathrm{s}), \delta 5.39$ $(2 \mathrm{H}, \mathrm{m}), \delta 6.04(1 \mathrm{H}, \mathrm{m}), \delta 6.49(1 \mathrm{H}, \mathrm{d}, J=3.6 \mathrm{~Hz}), \delta 6.77(1 \mathrm{H}, \mathrm{d}, J=3.6 \mathrm{~Hz}), \delta 7.03$ $(1 \mathrm{H}, \mathrm{d}, J=9.0 \mathrm{~Hz}), \delta 7.36(2 \mathrm{H}, \mathrm{m}), \delta 7.55(1 \mathrm{H}, \mathrm{td}, J=7.5,1.8 \mathrm{~Hz}), \delta 7.66(1 \mathrm{H}, \mathrm{dd}, J=$ 7.5, $1.2 \mathrm{~Hz}), \delta 8.54(1 \mathrm{H}, \mathrm{d}, J=9.0 \mathrm{~Hz}) ;{ }^{13} \mathrm{C} \mathrm{NMR}\left(75 \mathrm{MHz}, \mathrm{CDCl}_{3}\right) \delta 65.5,69.9,102.1$, $117.7,117.8,118.03,119.3,119.8,123.7,125.3,125.4,127.9,128.5,134.1,134.8$, 135.2; IR (neat, $\mathrm{cm}^{-1}$ ) 3077, 2985, 2340, 1887, 1645, 1555, 1321, 1220, 1057, 994, 861, 798, 752; HRMS calculated for $\mathrm{C}_{16} \mathrm{H}_{15} \mathrm{NO} 237.1154$ found 237.1158 . 

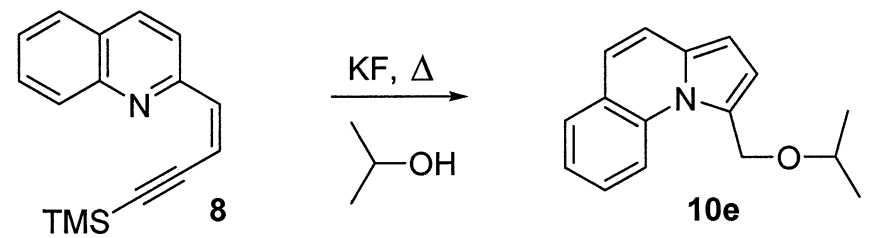

1-Isopropoxymethylpyrrolo [1,2-a] quinoline 10e: Compound 10e was synthesized according to general procedure A from compound $8(0.050 \mathrm{~g}, 0.20 \mathrm{mmol})$ and $\mathrm{KF}(0.023$ $\mathrm{g}, 0.40 \mathrm{mmol})$ in $20 \mathrm{~mL}$ of 2-propanol for $1.5 \mathrm{hr}$. Yield; $(0.041 \mathrm{~g}, 86 \%)$ as a yellow oil. ${ }^{1} \mathrm{H}$ NMR $\left(300 \mathrm{MHz}, \mathrm{CDCl}_{3}\right) \delta 1.22(6 \mathrm{H}, \mathrm{d}, J=1.2 \mathrm{~Hz}), \delta 3.87(1 \mathrm{H}, \mathrm{m}), \delta 4.96(2 \mathrm{H}, \mathrm{s}), \delta$ $6.48(1 \mathrm{H}, \mathrm{d}, J=3.7 \mathrm{~Hz}), \delta 6.75(1 \mathrm{H}, \mathrm{d}, J=3.7 \mathrm{~Hz}), \delta 7.00(1 \mathrm{H}, \mathrm{d}, J=9.0 \mathrm{~Hz}), \delta 7.34$ $(2 \mathrm{H}, \mathrm{m}), \delta 7.52(1 \mathrm{H}, \mathrm{td}, J=7.5,1.5 \mathrm{~Hz}), \delta 7.64(1 \mathrm{H}, \mathrm{dd}, J=7.5,1.5 \mathrm{~Hz}), \delta 8.56(1 \mathrm{H}, \mathrm{d}, J$ $=9.0 \mathrm{~Hz}) ;{ }^{13} \mathrm{C} \mathrm{NMR}\left(75 \mathrm{MHz}, \mathrm{CDCl}_{3}\right) \delta 22.4,63.9,70.0,102.1,117.5,118.0,119.3$, 119.7, 123.7, 125.3, 126.2, 127.7, 128.4, 134.0, 135.3; IR (neat, $\mathrm{cm}^{-1}$ ) 3051, 2973, 2871, 2359, 1608, 1555, 1367, 1124, 1045; MS (EI) m/z (rel. intensity) $239\left(\mathrm{M}^{+}, 45\right), 196(5)$, 180 (base), 179 (16), 168 (10), 152 (3), 128 (3); HRMS calculated for $\mathrm{C}_{16} \mathrm{H}_{17} \mathrm{NO}$ 239.1302, found 239.1302.
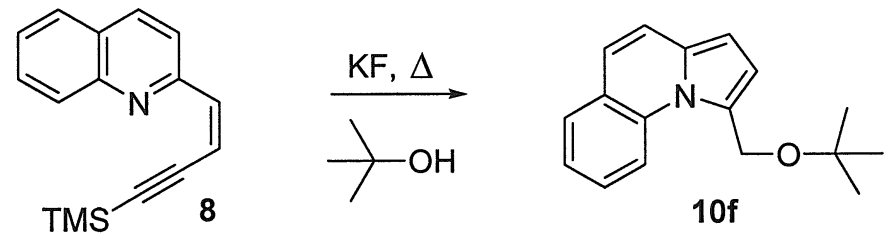

1-tert-Butoxymethylpyrrolo [1,2-a] quinoline 10f: Compound $10 \mathrm{f}$ was synthesized according to general procedure A from compound $8(0.050 \mathrm{~g}, 0.20 \mathrm{mmol})$ and $\mathrm{KF}(0.023$ $\mathrm{g}, 0.40 \mathrm{mmol})$ in $20 \mathrm{~mL}$ of tert-butanol for $7 \mathrm{hrs}$. Yield; (0.052 g, 97\%) as a yellow solid; $\mathrm{mp}=45-47^{\circ} \mathrm{C} .{ }^{1} \mathrm{H}$ NMR $\left(300 \mathrm{MHz}, \mathrm{CDCl}_{3}\right) \delta 1.32(9 \mathrm{H}, \mathrm{s}), \delta 4.81(2 \mathrm{H}, \mathrm{s}), \delta 6.40(1 \mathrm{H}$, $\mathrm{d}, J=3.7 \mathrm{~Hz}), \delta 6.66(1 \mathrm{H}, \mathrm{d}, J=3.7 \mathrm{~Hz}), \delta 6.90(1 \mathrm{H}, \mathrm{d}, J=9.0 \mathrm{~Hz}), \delta 7.26(2 \mathrm{H}, \mathrm{m}), \delta$ 
$7.43(1 \mathrm{H}, \mathrm{td}, J=7.2,1.8 \mathrm{~Hz}), \delta 7.56(1 \mathrm{H}, \mathrm{d}, J=1.8 \mathrm{~Hz}), \delta 8.53(1 \mathrm{H}, \mathrm{d}, J=9.0 \mathrm{~Hz}) ;{ }^{13} \mathrm{C}$ $\operatorname{NMR}\left(75 \mathrm{MHz}, \mathrm{CDCl}_{3}\right) \delta 28.1,58.5,74.14,102.3,116.8,118.1,119.4,119.4,123.6$, 125.3, 127.1, 127.3, 128.4, 133.7, 135.2; HRMS calculated for $\mathrm{C}_{17} \mathrm{H}_{19} \mathrm{NO} 253.1467$ found 253.1470 .
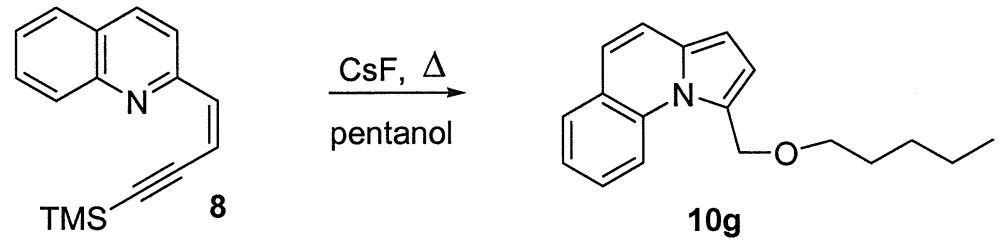

1-Pentyloxymethylpyrrolo [1,2-a] quinoline 10g: Synthesized according to general procedure B from protected enyne $8(0.050 \mathrm{~g}, 0.20 \mathrm{mmol})$ and $\mathrm{CsF}(0.045 \mathrm{~g}, 0.30 \mathrm{mmol})$ in $20 \mathrm{~mL}$ of pentanol for $1 \mathrm{hr}$. Yield; $0.030 \mathrm{~g},(57 \%)$ as a yellow oil. ${ }^{1} \mathrm{H}$ NMR $(300 \mathrm{MHz}$, $\left.\mathrm{CDCl}_{3}\right) \delta 0.88(3 \mathrm{H}, \mathrm{t}, J=7.5 \mathrm{~Hz}), \delta 1.36(4 \mathrm{H}, \mathrm{m}), \delta 1.65(2 \mathrm{H}, \mathrm{m}), \delta 3.61(2 \mathrm{H}, \mathrm{t}, J=6.6$ $\mathrm{Hz}), \delta 4.94(2 \mathrm{H}, \mathrm{s}), \delta 6.49(1 \mathrm{H}, \mathrm{d}, J=3.8 \mathrm{~Hz}), \delta 6.77(1 \mathrm{H}, \mathrm{d}, J=3.8 \mathrm{~Hz}), \delta 7.02(1 \mathrm{H}, \mathrm{d}, J$ $=9.0 \mathrm{~Hz}), \delta 7.36(2 \mathrm{H}, \mathrm{m}), \delta 7.51(1 \mathrm{H}, \mathrm{td}, J=7.8,1.5 \mathrm{~Hz}), \delta 7.66(1 \mathrm{H}, \mathrm{dd} J=7.8,1.5$ $\mathrm{Hz}), \delta 8.50(1 \mathrm{H}, \mathrm{d}, J=9.0 \mathrm{~Hz}) ;{ }^{13} \mathrm{C} \mathrm{NMR}\left(75 \mathrm{MHz}, \mathrm{CDCl}_{3}\right) \delta 14.2,22.7,28.8,29.6$, $66.4,69.5,102.0,117.7,117.9,119.3,119.7,123.7,125.3,125.9,127.8,128.4,133.9$, 135.2; IR (in $\mathrm{CHCl}_{3}, \mathrm{~cm}^{-1}$ ) 2871, 1631, 1478, 1424, 1322, 1123, 1085, 908, 876, 799, 737; HRMS calculated for $\mathrm{C}_{18} \mathrm{H}_{21} \mathrm{NO} 267.1623$ found 267.1625 . 


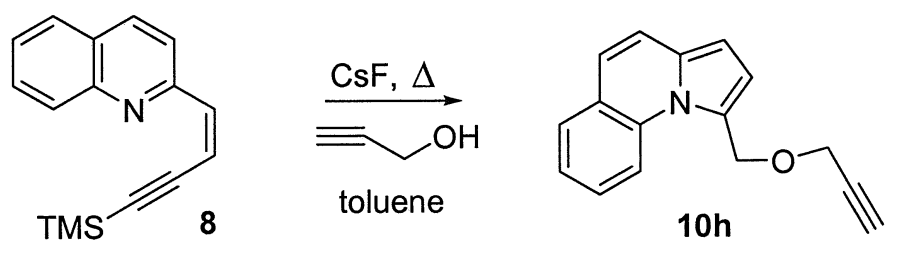

1-Prop-2-ynyloxymethylpyrrolo [1,2-a] quinoline 10h: Synthesized according to general procedure C from silylated enyne 8 (0.050 g, $0.20 \mathrm{mmol}), \mathrm{CsF}(0.045 \mathrm{~g}, 0.29$ $\mathrm{mmol})$ and propargyl alcohol $(0.112 \mathrm{~g}, 2.0 \mathrm{mmol})$ in $20 \mathrm{~mL}$ of anhydrous toluene for 3 hrs. Yield; $0.024 \mathrm{~g}(51 \%)$ as a yellow oil. ${ }^{1} \mathrm{H}$ NMR $\left(500 \mathrm{MHz}, \mathrm{CDCl}_{3}\right) \delta 2.52(1 \mathrm{H}, \mathrm{t}, J=$ $2.5 \mathrm{~Hz}), 4.25(2 \mathrm{H}, \mathrm{d}, J=2.5 \mathrm{~Hz}), \delta 5.09(2 \mathrm{H}, \mathrm{s}), \delta 6.49(1 \mathrm{H}, \mathrm{d}, J=3.8 \mathrm{~Hz}), \delta 6.82(1 \mathrm{H}$, $\mathrm{d}, J=3.8 \mathrm{~Hz}), \delta 7.02(1 \mathrm{H}, \mathrm{d}, J=9.0 \mathrm{~Hz}), \delta 7.34(2 \mathrm{H}, \mathrm{m}), \delta 7.52(1 \mathrm{H}, \mathrm{td}, J=7.5,1.8 \mathrm{~Hz})$, $\delta 7.65(1 \mathrm{H}, \mathrm{dd}, J=7.5,1.8 \mathrm{~Hz}), \delta 8.49(1 \mathrm{H}, \mathrm{d}, J=9.0 \mathrm{~Hz}) ;{ }^{13} \mathrm{C} \mathrm{NMR}\left(75 \mathrm{MHz}, \mathrm{CDCl}_{3}\right)$ $\delta 55.9,64.8,75.1,79.8,102.2,117.8,118.8,119.3,120.1,123.8,124.3,125.3,128.0$ 128.6, 134.4, 135.1; IR (neat, $\mathrm{cm}^{-1}$ ) 3292, 2848, 2248, 2116, 1732, 1608, 1472, 1322 , 1123, 1063, 912, 800, 730; HRMS calculated for $\mathrm{C}_{16} \mathrm{H}_{13} \mathrm{NO} 235.0997$ found 235.1000.

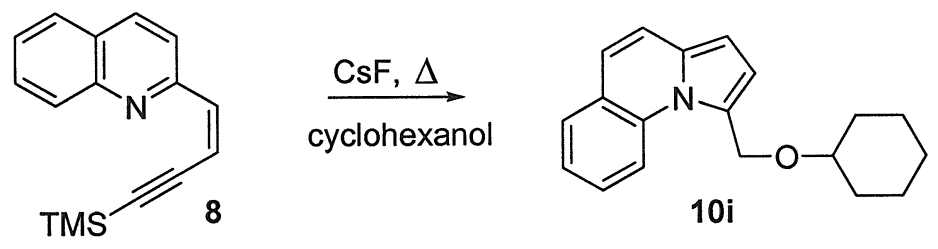

1-Cyclohexyloxymethylpyrrolo [1,2-a] quinoline 10i: Compound 10i was synthesized according to general procedure $\mathrm{B}$ from compound $8(0.050 \mathrm{~g}, 0.20 \mathrm{mmol})$ and $\mathrm{CsF}(0.045$ $\mathrm{g}, 0.29 \mathrm{mmol})$ in $20 \mathrm{~mL}$ of cyclohexanol for $1 \mathrm{hr}$. Yield; $0.043 \mathrm{~g},(77 \%)$ as yellow oil. ${ }^{1} \mathrm{H}$ NMR (300 MHz, $\left.\mathrm{CDCl}_{3}\right) \delta 1.25(3 \mathrm{H}, \mathrm{m}), \delta 1.38(2 \mathrm{H}, \mathrm{m}), 1.54(1 \mathrm{H}, \mathrm{m}), \delta 1.78(2 \mathrm{H}, \mathrm{m}), \delta$ $1.99(2 \mathrm{H}, \mathrm{m}), \delta 3.56(1 \mathrm{H}, \mathrm{m}), 4.98(2 \mathrm{H}, \mathrm{s}), \delta 6.47(1 \mathrm{H}, \mathrm{d}, J=3.9 \mathrm{~Hz}), \delta 6.74(1 \mathrm{H}, \mathrm{d}, J=$ $3.9 \mathrm{~Hz}), \delta 6.99(1 \mathrm{H}, \mathrm{d}, J=9.3 \mathrm{~Hz}), \delta 7.33(2 \mathrm{H}, \mathrm{m}), \delta 7.52(1 \mathrm{H}, \mathrm{td}, J=7.2,1.5 \mathrm{~Hz}), \delta 7.64$ 
$(1 \mathrm{H}, \mathrm{dd}, J=7.2,1.5 \mathrm{~Hz}), \delta 8.59(1 \mathrm{H}, \mathrm{d}, J=9.3 \mathrm{~Hz}) ;{ }^{13} \mathrm{C} \mathrm{NMR}\left(75 \mathrm{MHz}, \mathrm{CDCl}_{3}\right) \delta 24.5$, $26.1,32.5,63.5,76.1,102.1,117.5,118.1,119.3,119.6,123.6,125.3,126.4,127.6$, 128.4, 133.9, 135.2; IR (neat, $\mathrm{cm}^{-1}$ ) 3049, 2833, 1607, 1553, 1424, 1360, 1220, 1122 , 1071, 949, 875, 796; HRMS calculated for $\mathrm{C}_{19} \mathrm{H}_{21} \mathrm{NO} 279.1623$ found 279.1630.
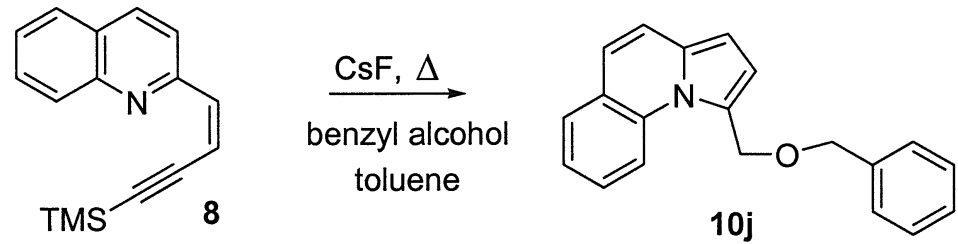

1-Benzyloxymethylpyrrolo [1,2-a] quinoline $10 \mathrm{j}$ : Compound $10 \mathrm{j}$ was synthesized according to general procedure $\mathrm{C}$ from compound $8(0.030 \mathrm{~g}, 0.12 \mathrm{mmol})$ and $\mathrm{CsF}(0.027$ $\mathrm{g}, 0.18 \mathrm{mmol})$, and benzyl alcohol $(0.129 \mathrm{~g}, 1.2 \mathrm{mmol})$ in $20 \mathrm{~mL}$ of dry toluene for $2 \mathrm{hrs}$. The residue was purified by flash column chromatography (silica gel, 15:1 hexanes/ethyl acetate) to yield, $0.016 \mathrm{~g}$, (47\%) as a yellow oil. ${ }^{1} \mathrm{H} \mathrm{NMR}\left(300 \mathrm{MHz}, \mathrm{CDCl}_{3}\right) \delta 4.64(2 \mathrm{H}$, s), $\delta 5.01(2 \mathrm{H}, \mathrm{s}), \delta 6.48(1 \mathrm{H}, \mathrm{d}, J=3.9 \mathrm{~Hz}), \delta 6.75(1 \mathrm{H}, \mathrm{d}, J=3.9 \mathrm{~Hz}), \delta 7.02(1 \mathrm{H}, \mathrm{d}, J=$ $9.0 \mathrm{~Hz}), \delta 7.46(7 \mathrm{H}, \mathrm{m}), \delta 7.49(1 \mathrm{H}, \mathrm{td}, J=7.5,1.8 \mathrm{~Hz}), \delta 7.65(1 \mathrm{H}, \mathrm{dd}, J=7.5,1.8 \mathrm{~Hz})$, $\delta 8.53(1 \mathrm{H}, \mathrm{d}, J=9.0 \mathrm{~Hz}) ;{ }^{13} \mathrm{C} \mathrm{NMR}\left(75 \mathrm{MHz}, \mathrm{CDCl}_{3}\right) \delta 65.6,71.1,102.1,117.8,118.2$, $119.3,119.9,123.7,125.3,125.4,127.9,127.9,128.4,127.5,128.7,134.2,135.2,138.2$

IR (in $\mathrm{CHCl}_{3}, \mathrm{~cm}^{-1}$ ) 3030, 2848, 2245, 1731, 1606, 1555, 1472, 1309, 1123, 1061, 910, 730; HRMS calculated for $\mathrm{C}_{20} \mathrm{H}_{17} \mathrm{NO} 287.1310$ found 287.1320 . 
References:

1. (a) Uenishi, J.; Kawahama, R.; Yonemitsu, O.; Tsuji, J. Tetrahedron Lett. 1996, 37, 6759-6762. (b) Uenishi, J.; Kawahama, R.; Yonemitsu, O.; Tsuji, J. J. Org. Chem. 1998, 63, 8965-8975. (c) Uenishi, J.; Kawahama, R.; Yonemitsu, O.; Tsuji, J. J. Org. Chem. 1996, 61, 5716-5717.

2. Eberbach, W.; Maier, W. Tetrahedron Lett. 1989, 30, 5591-5594.

3. Hayford, A.; Kaloko, J. Jr. US. Pat. Appl. 60/640,369, 2004. 


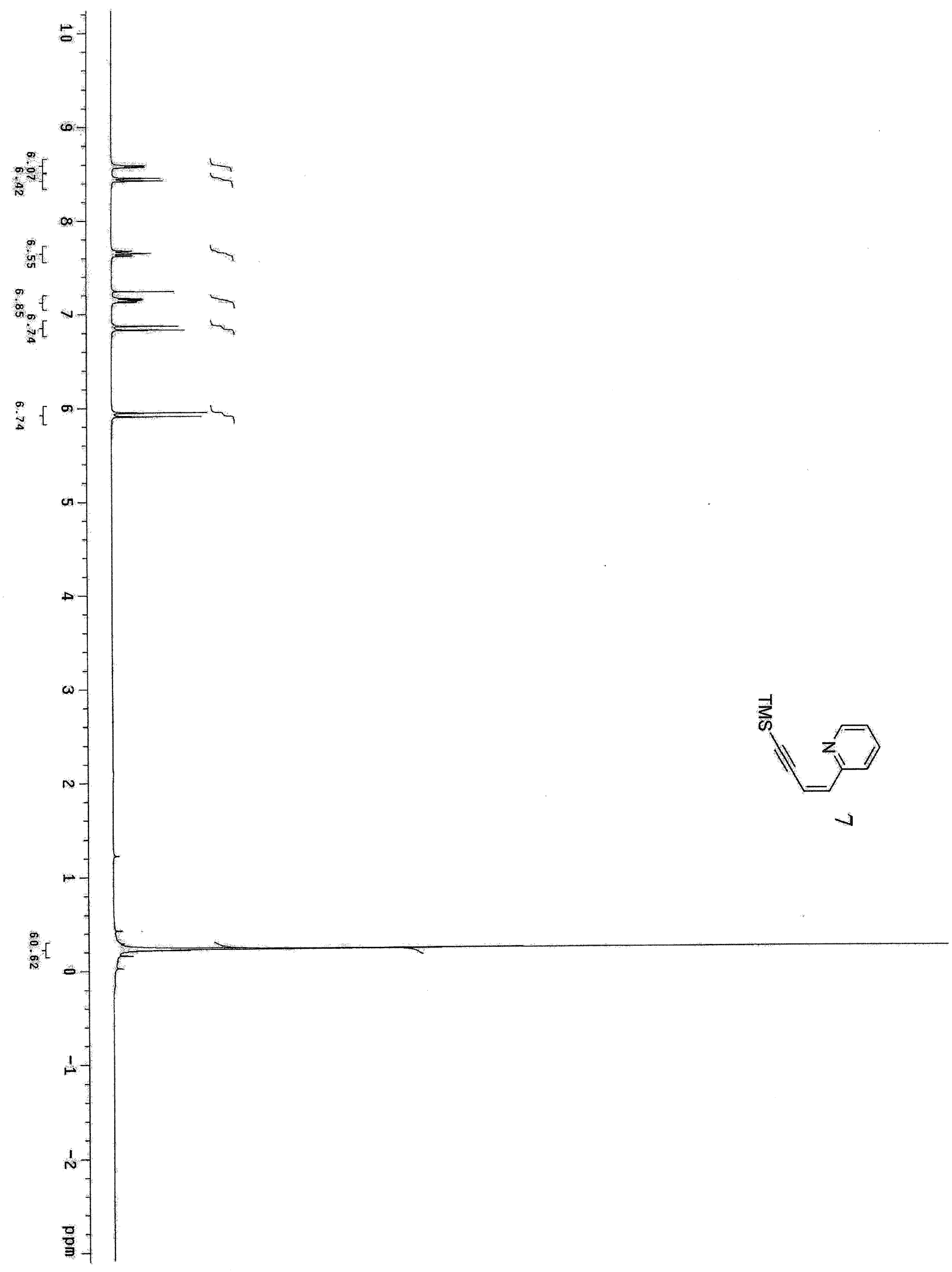



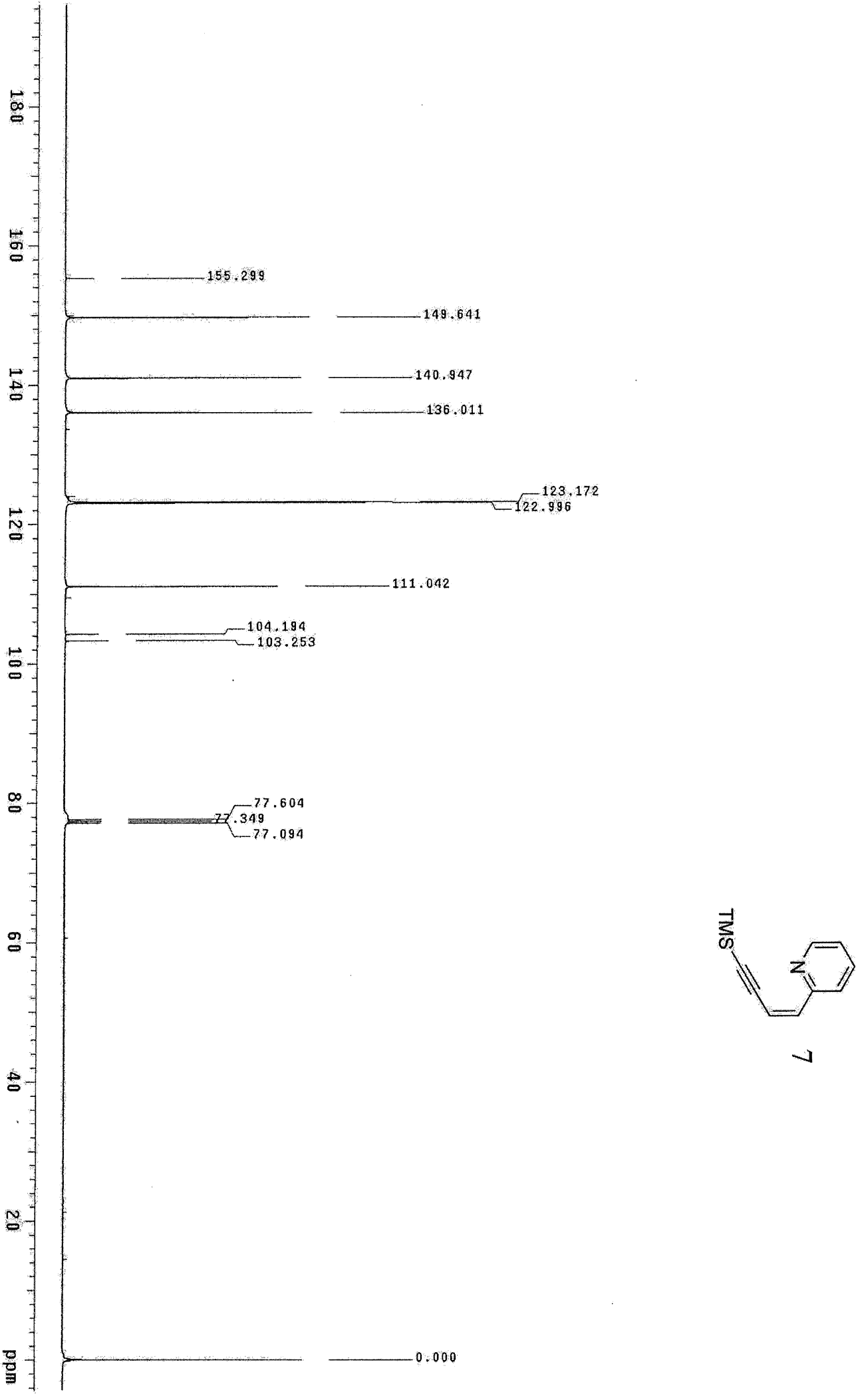
SI 22

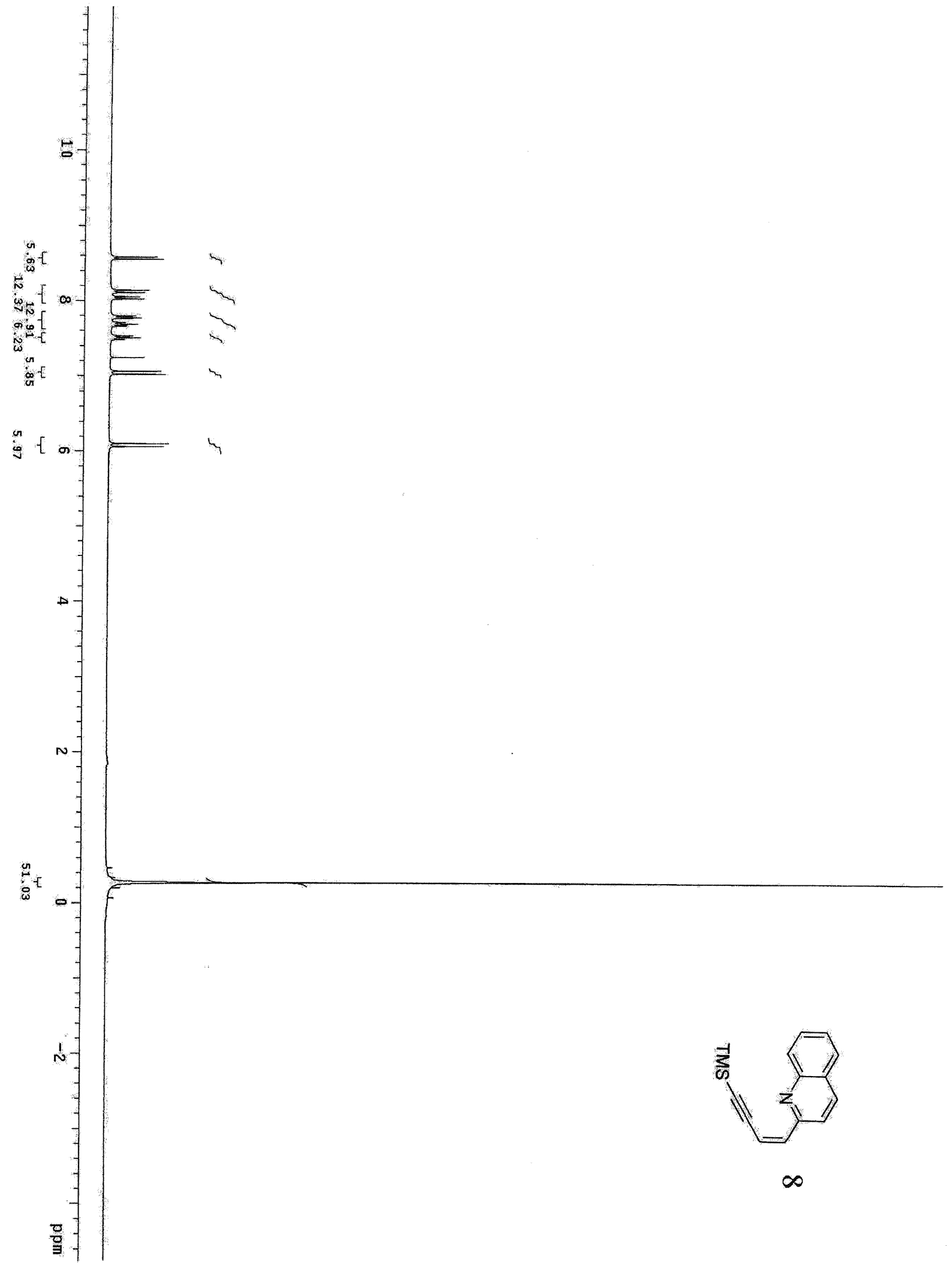



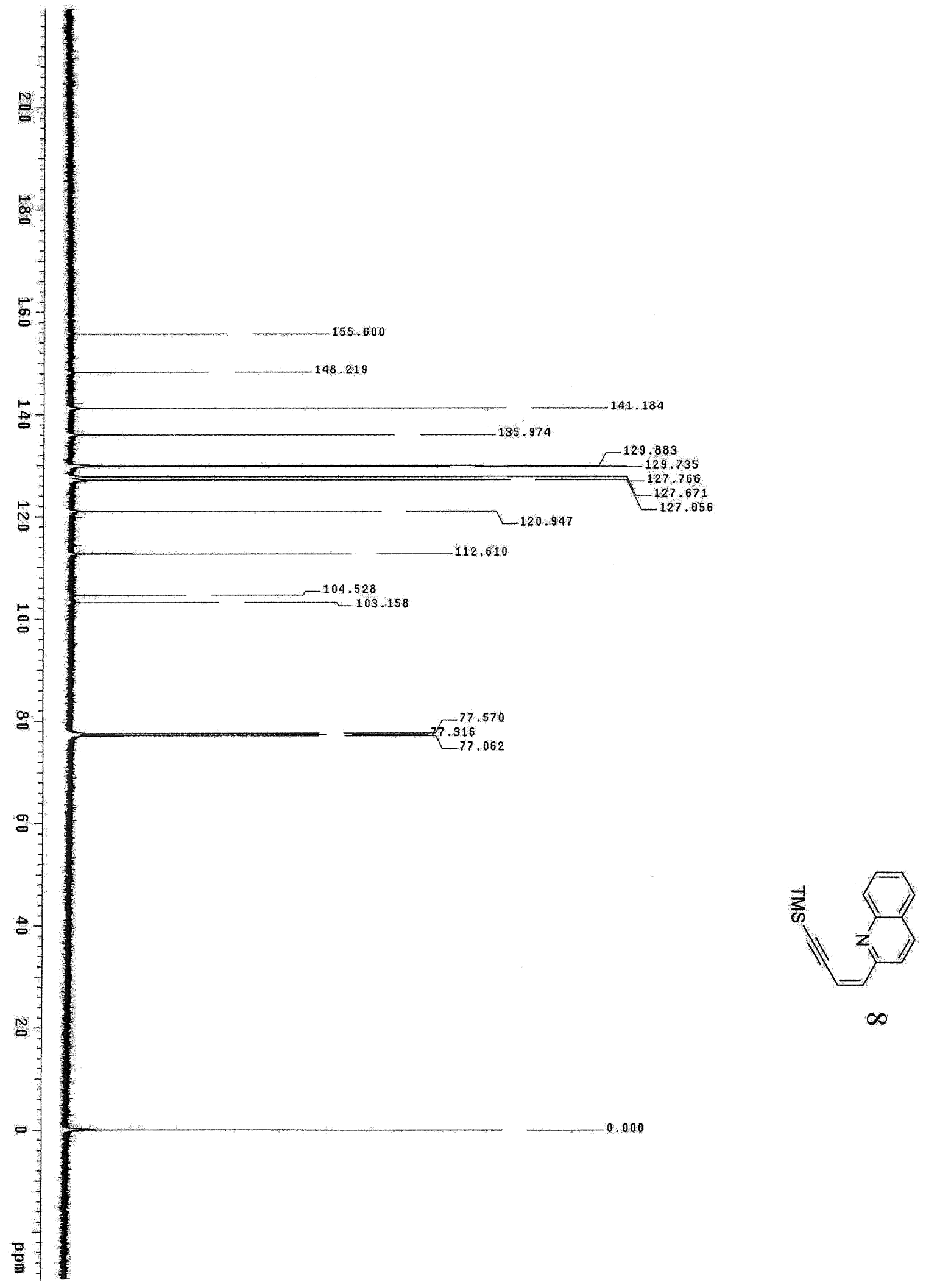

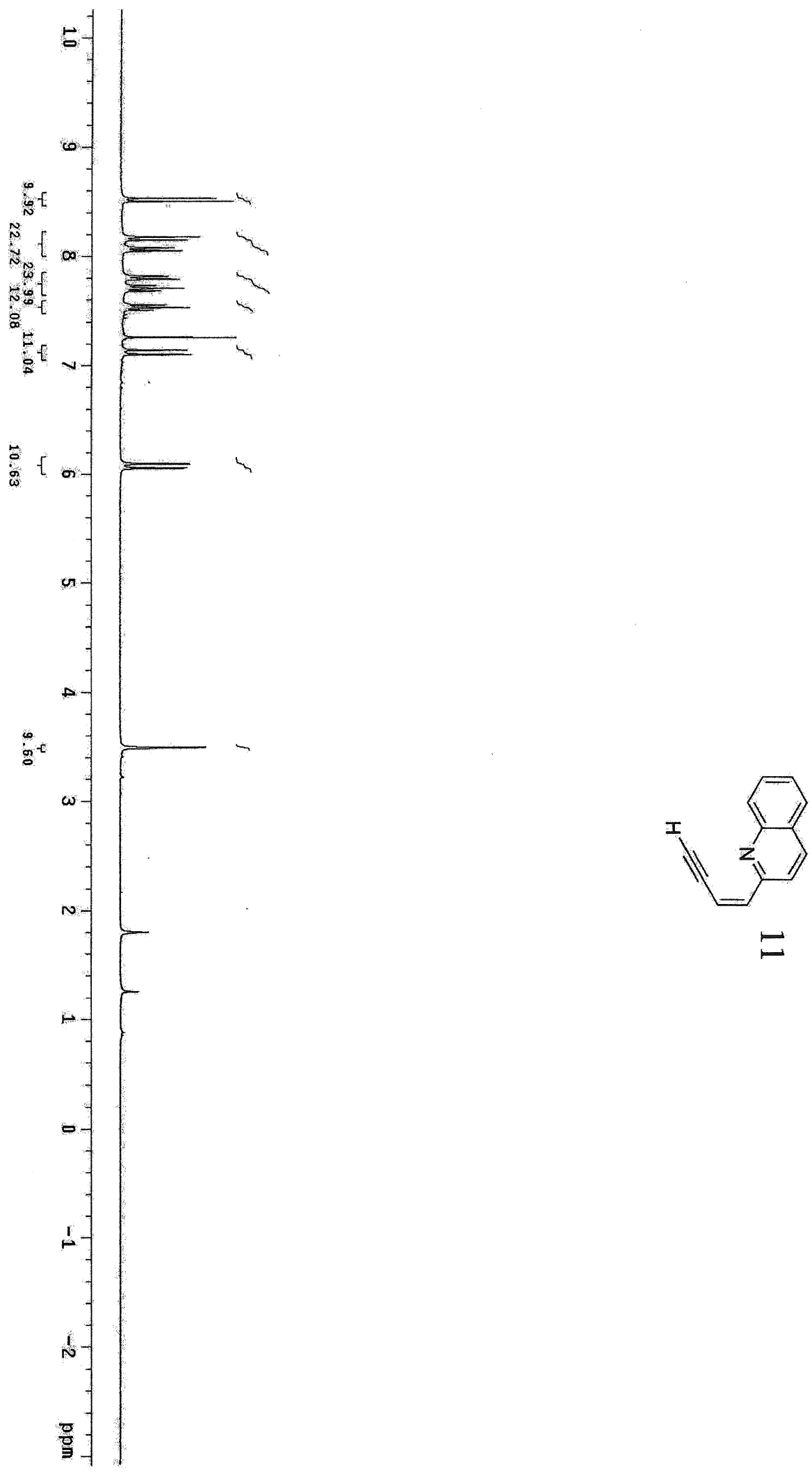


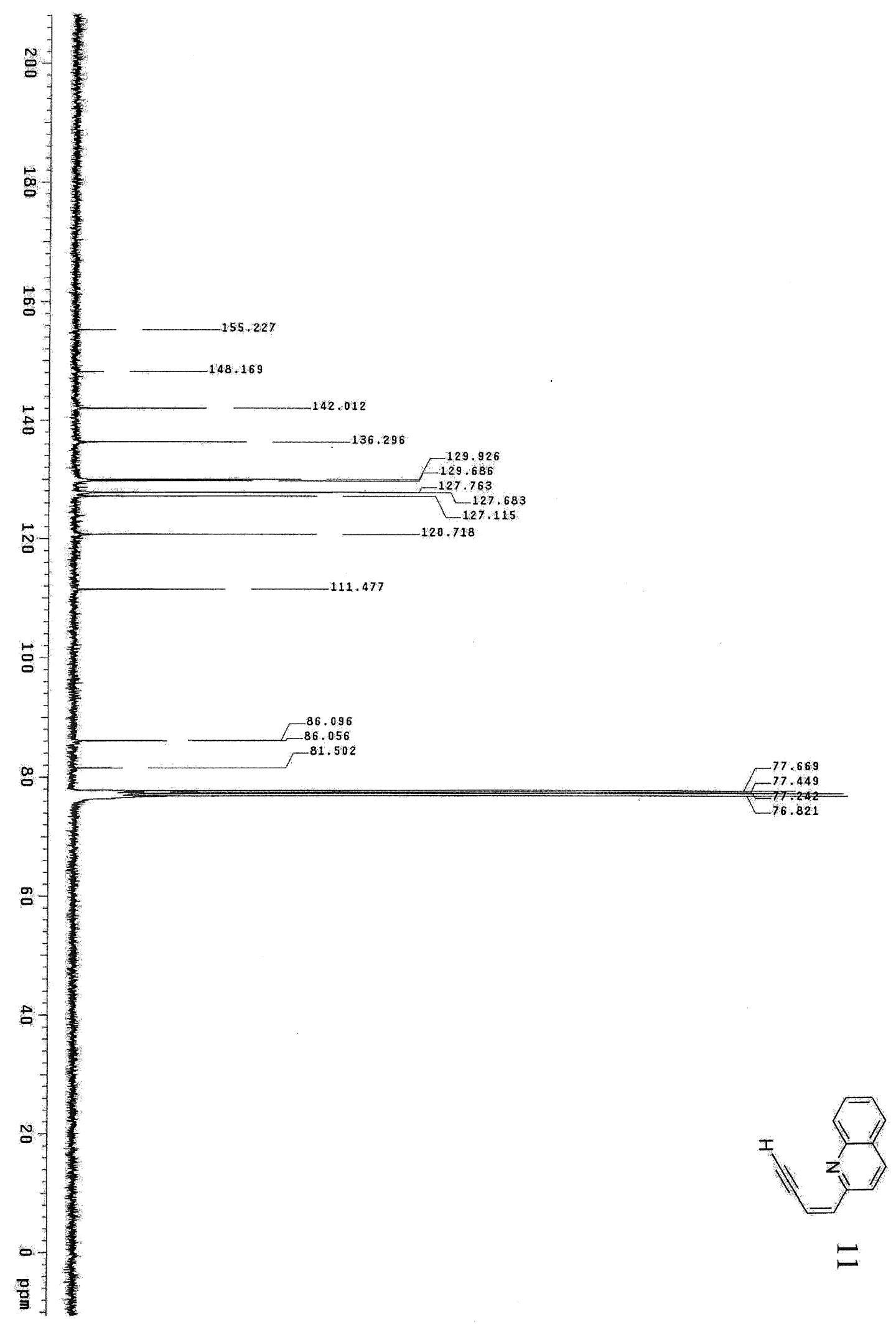




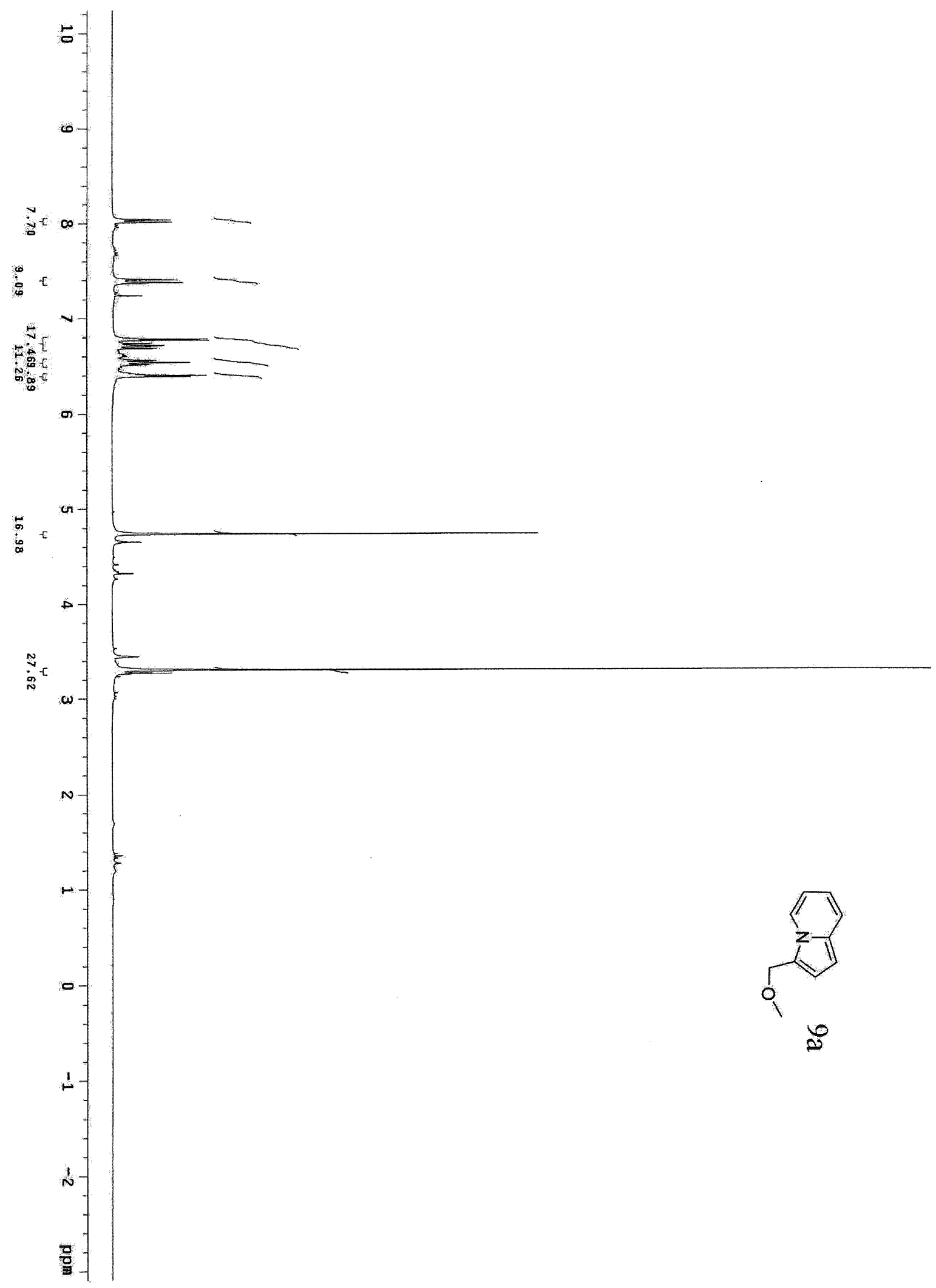



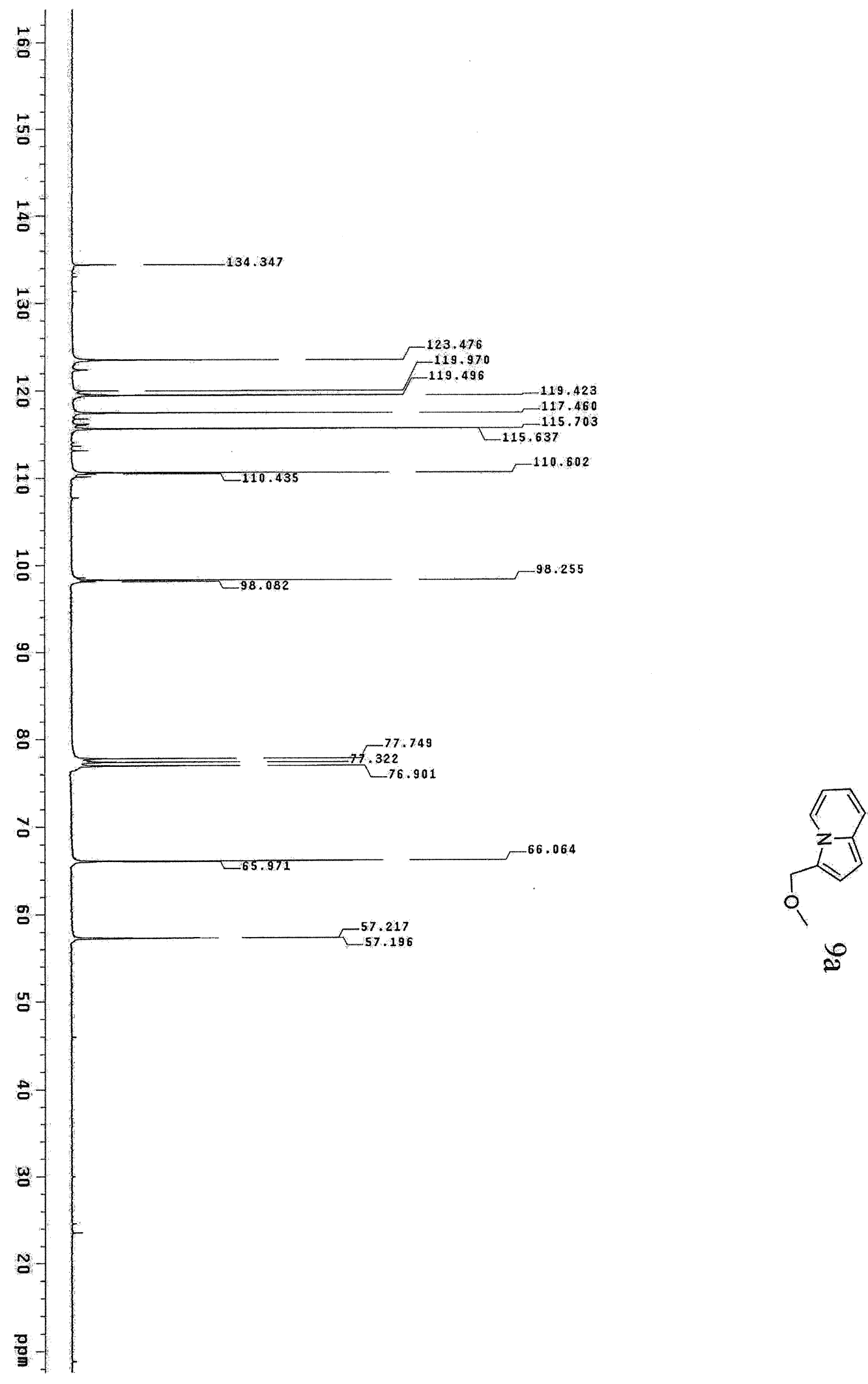

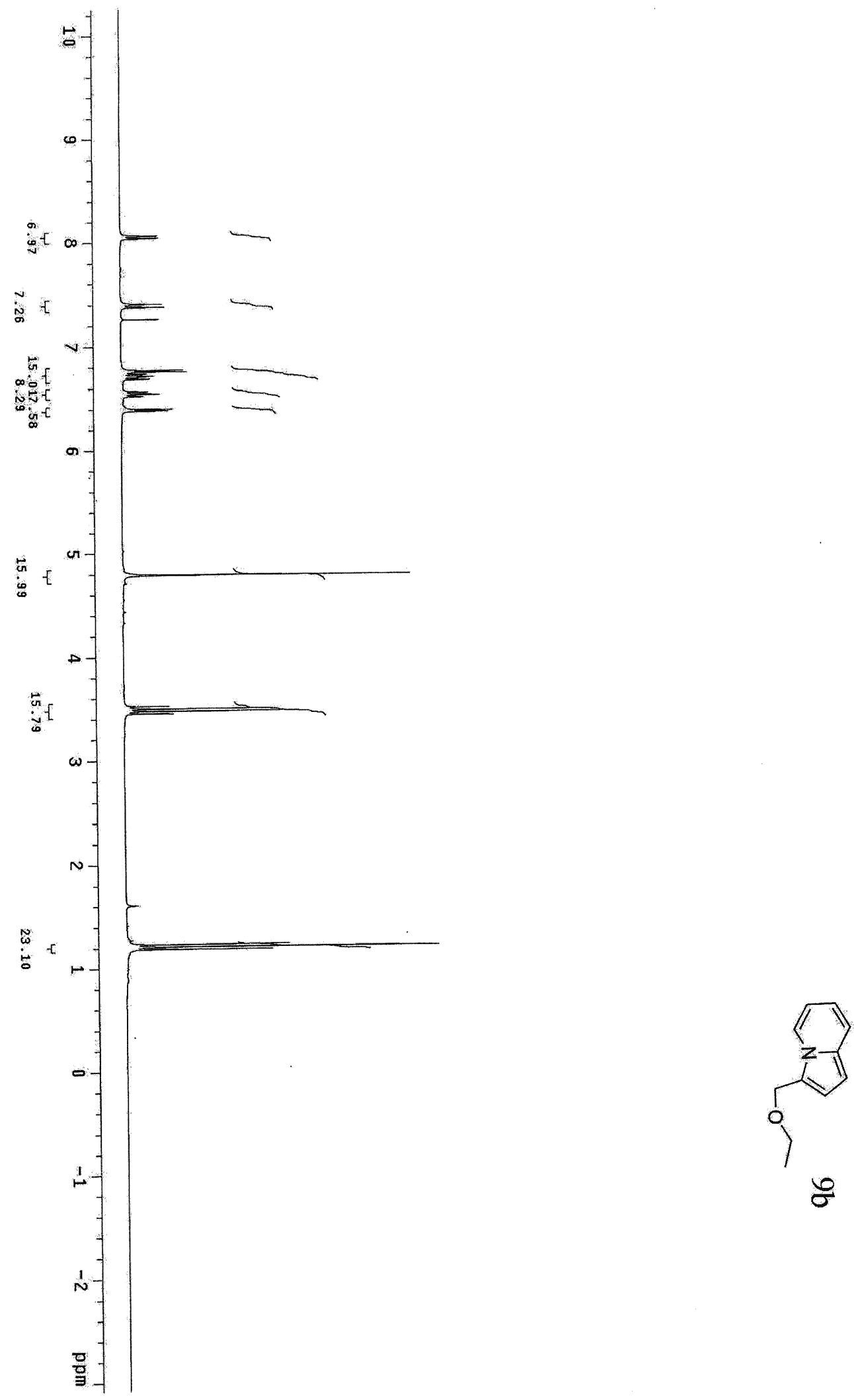

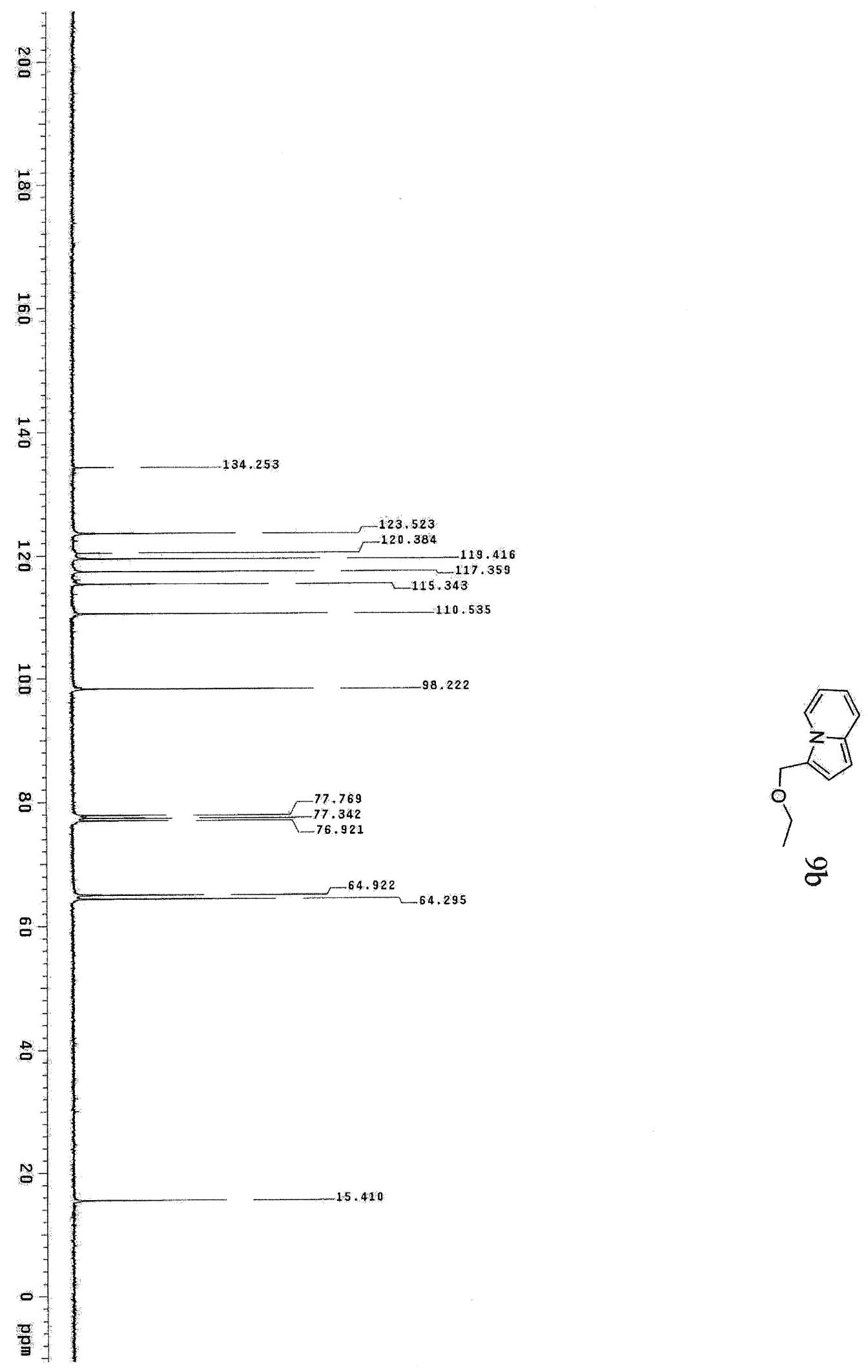

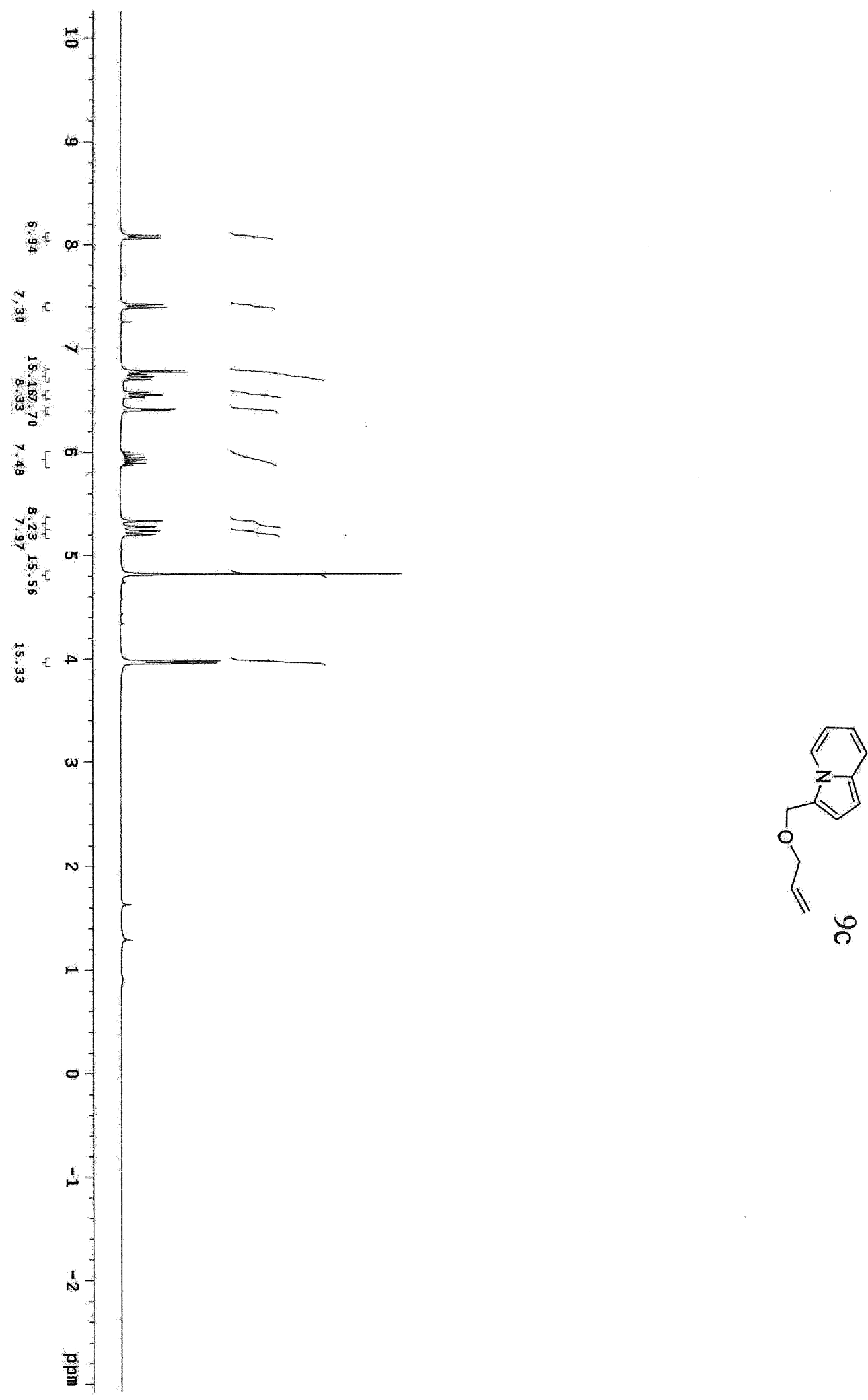

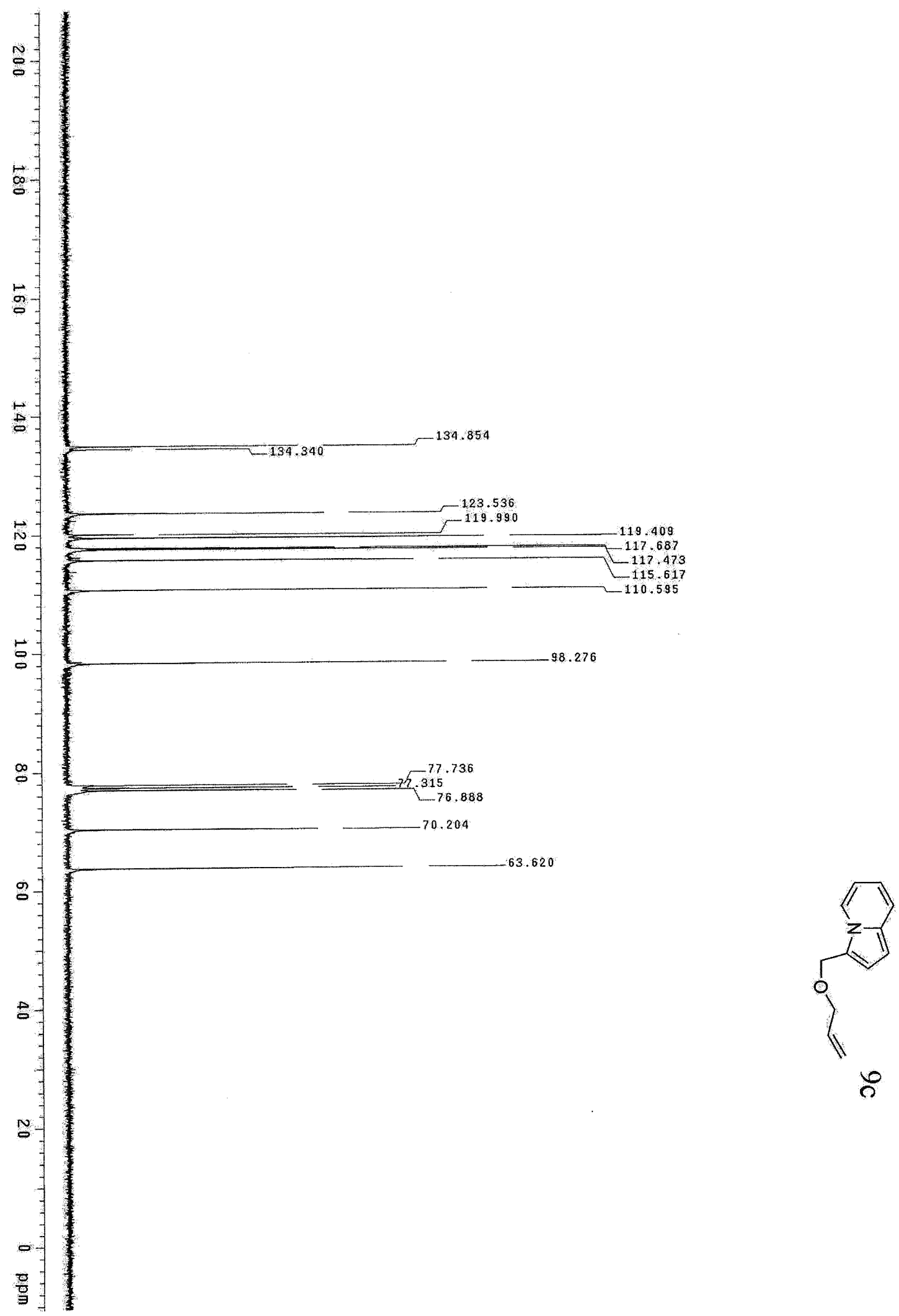

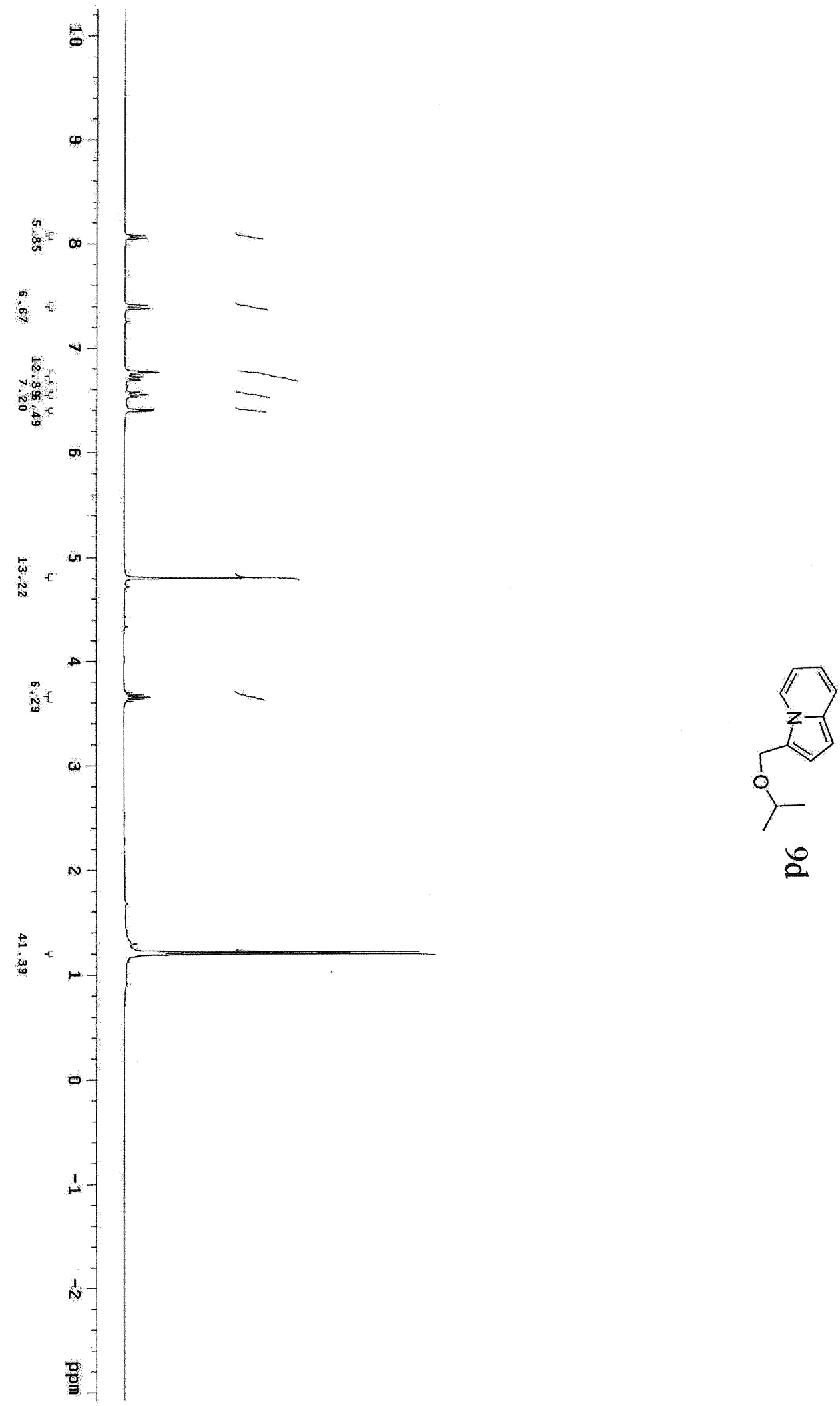

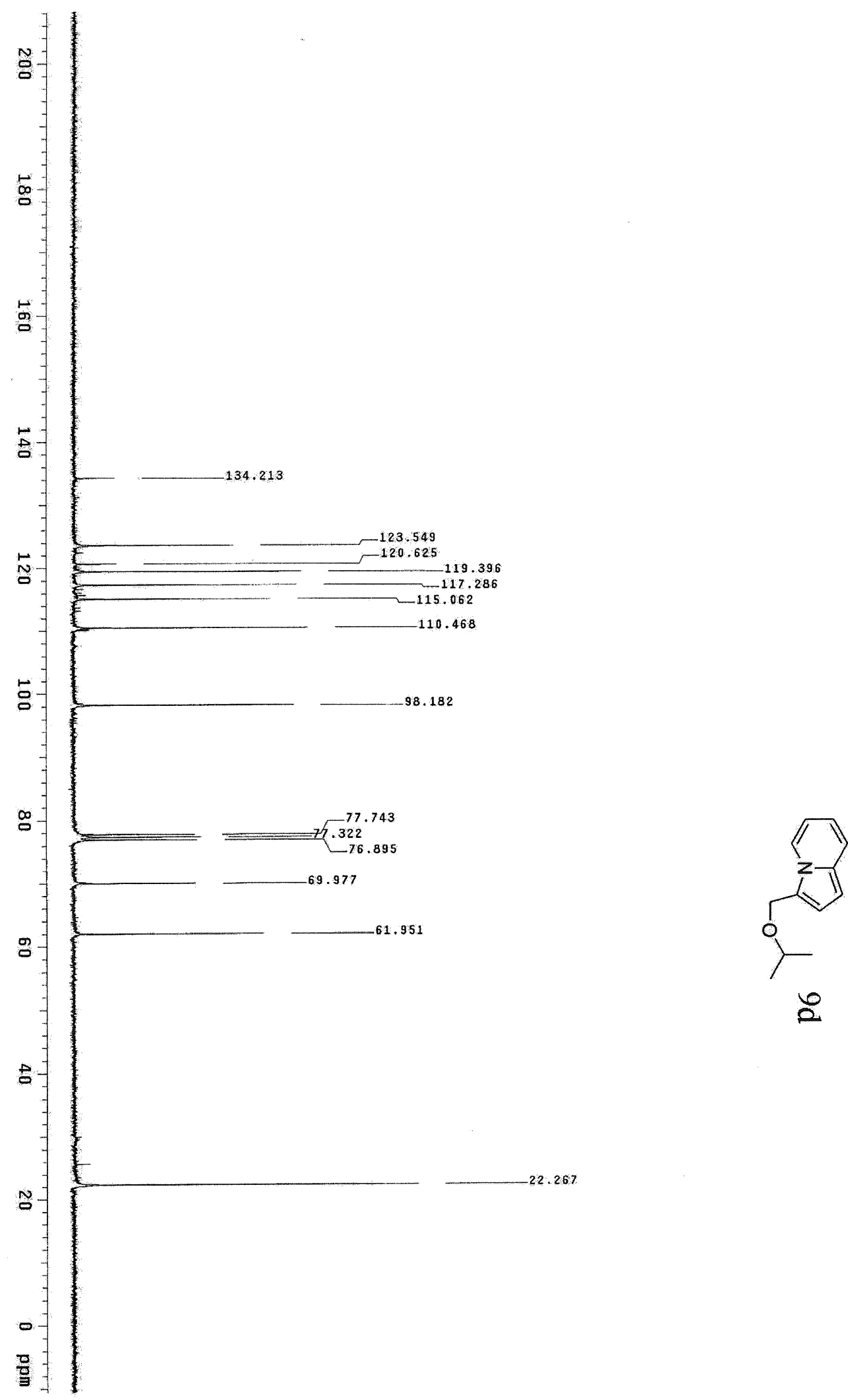


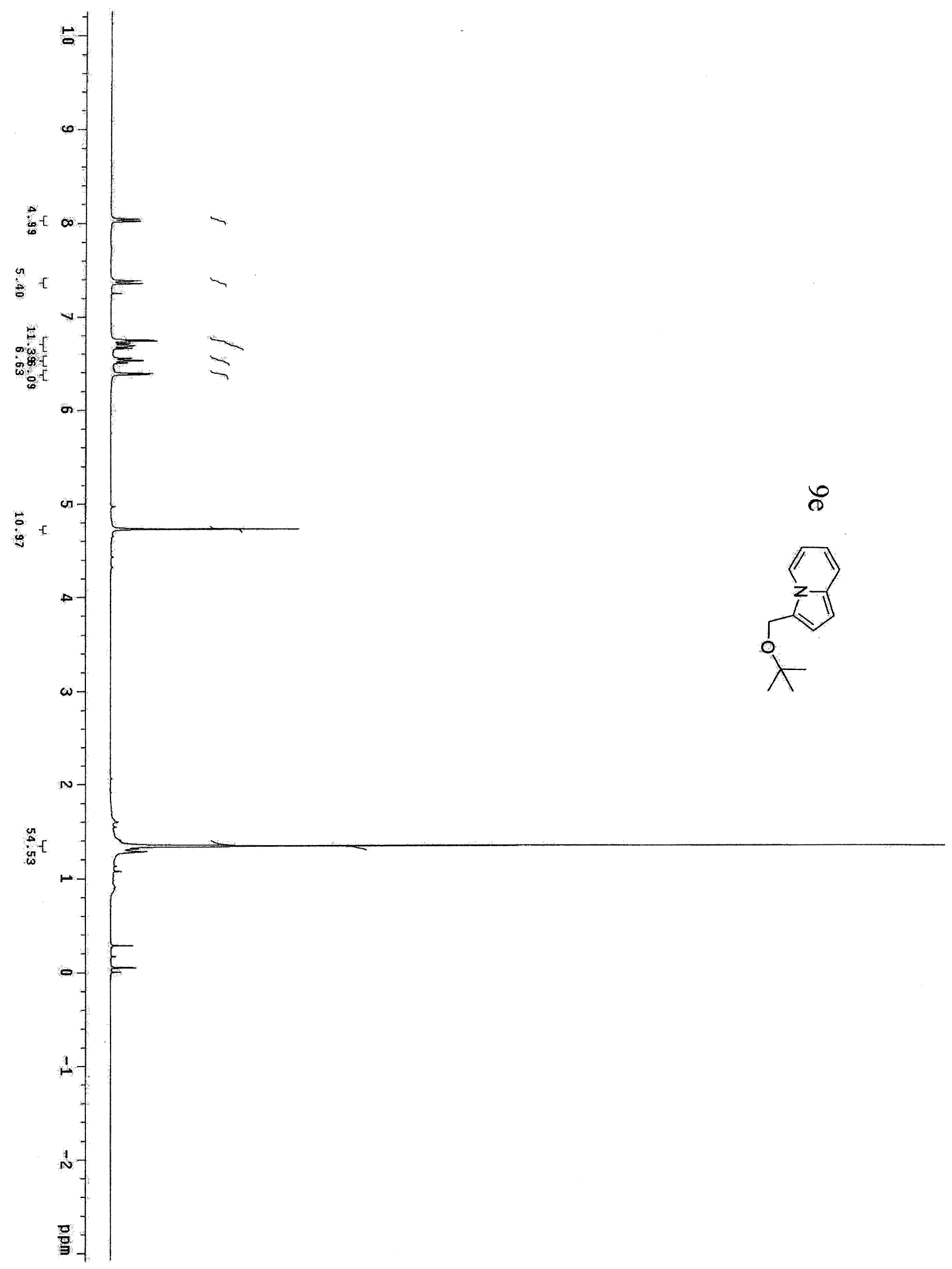



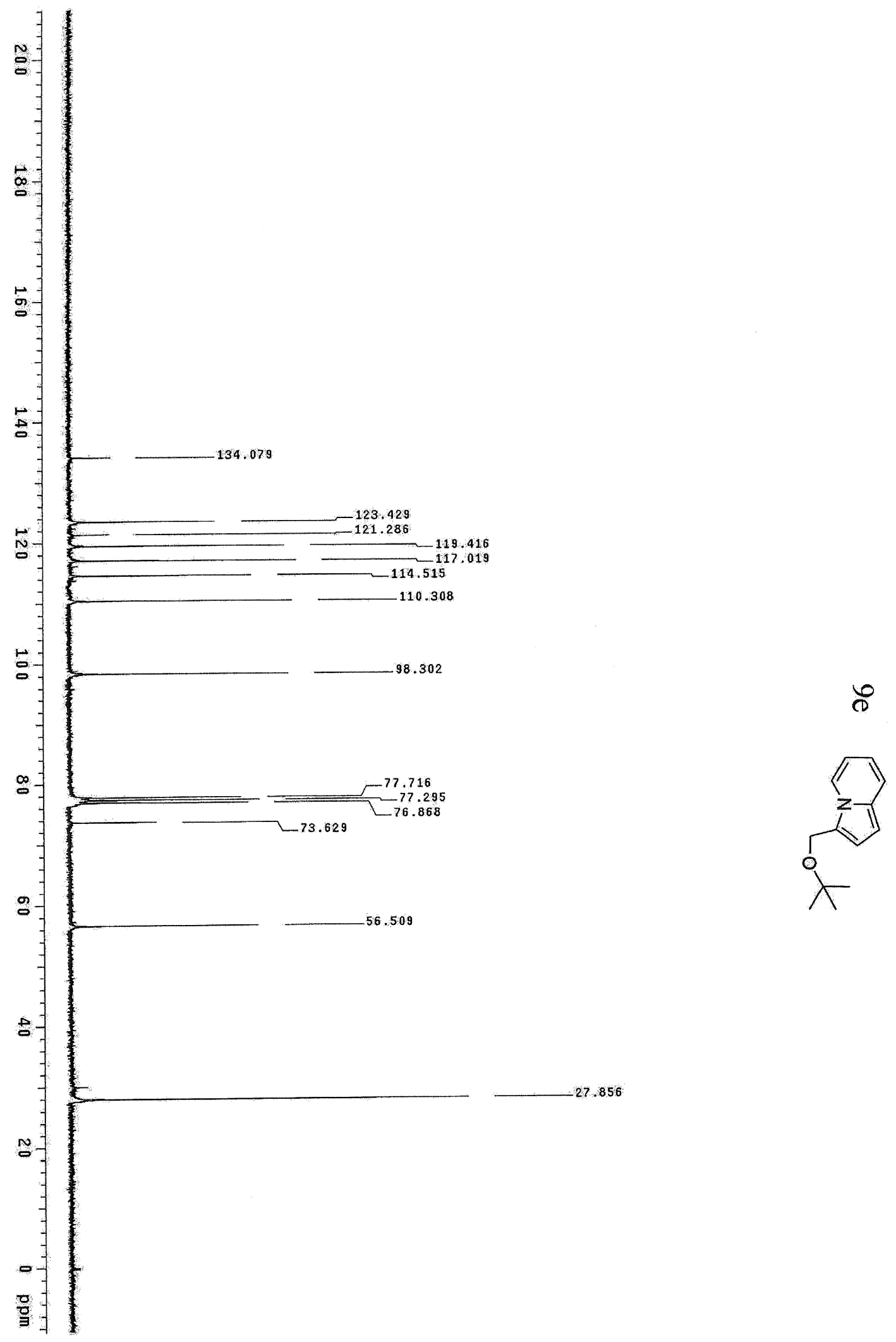

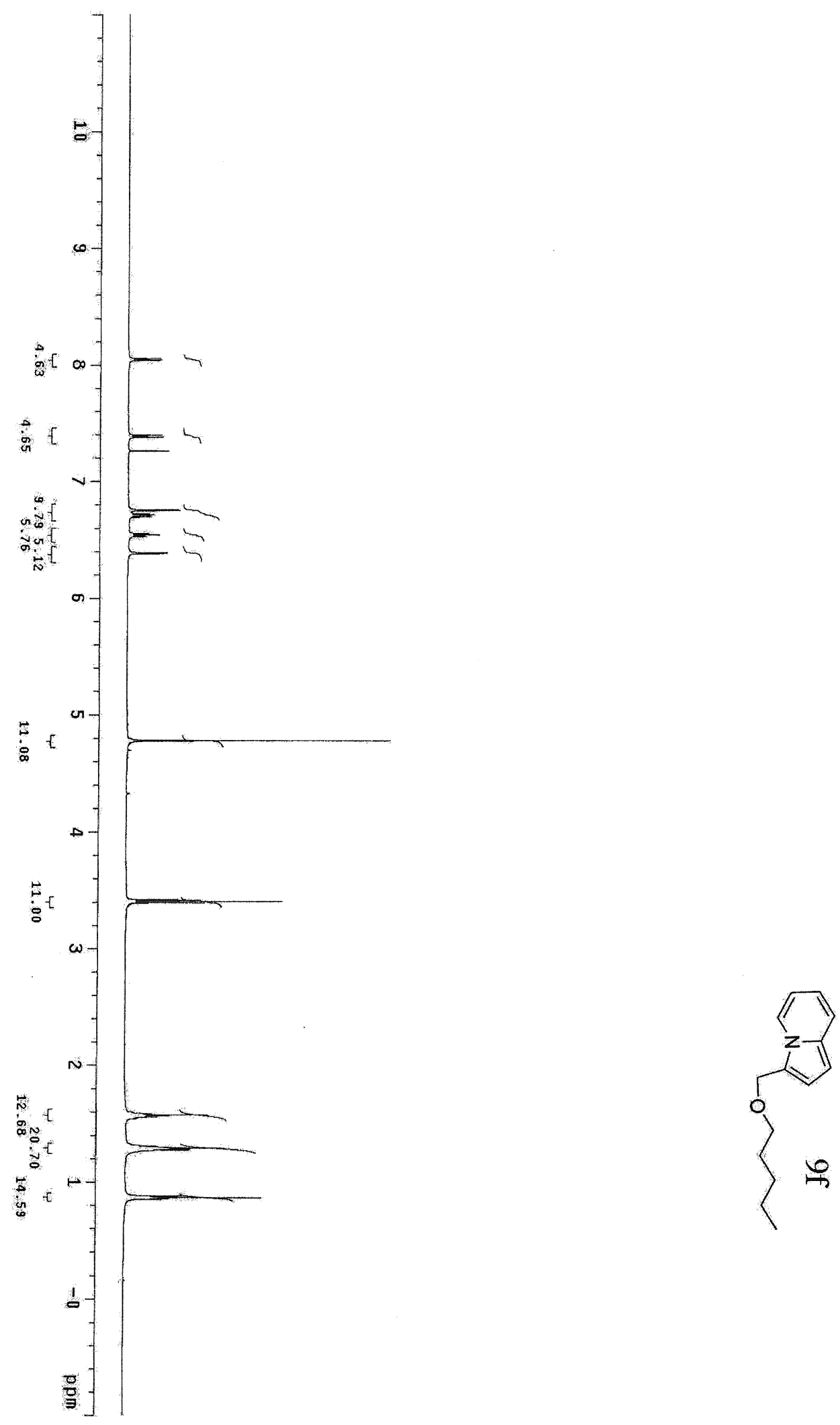

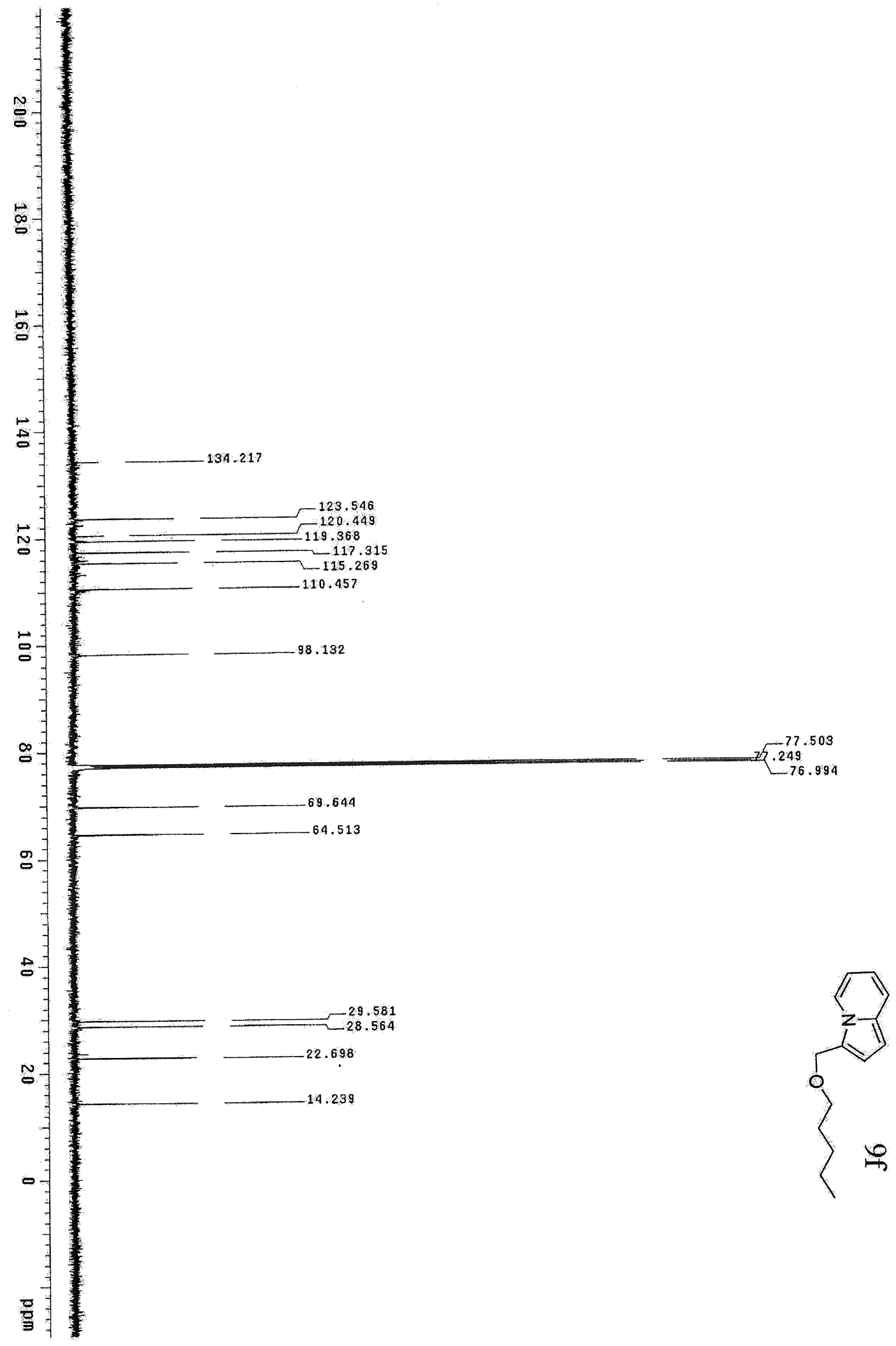

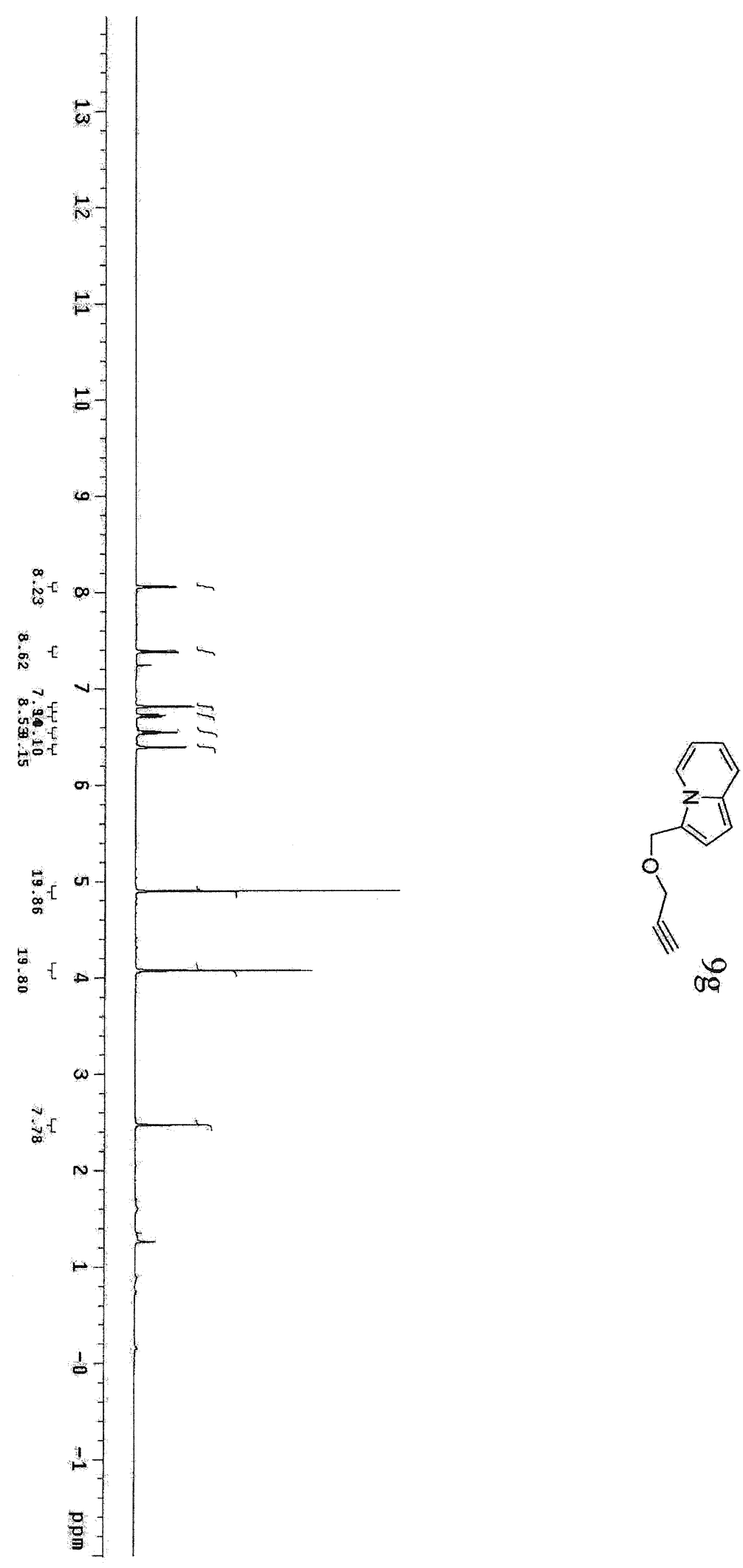


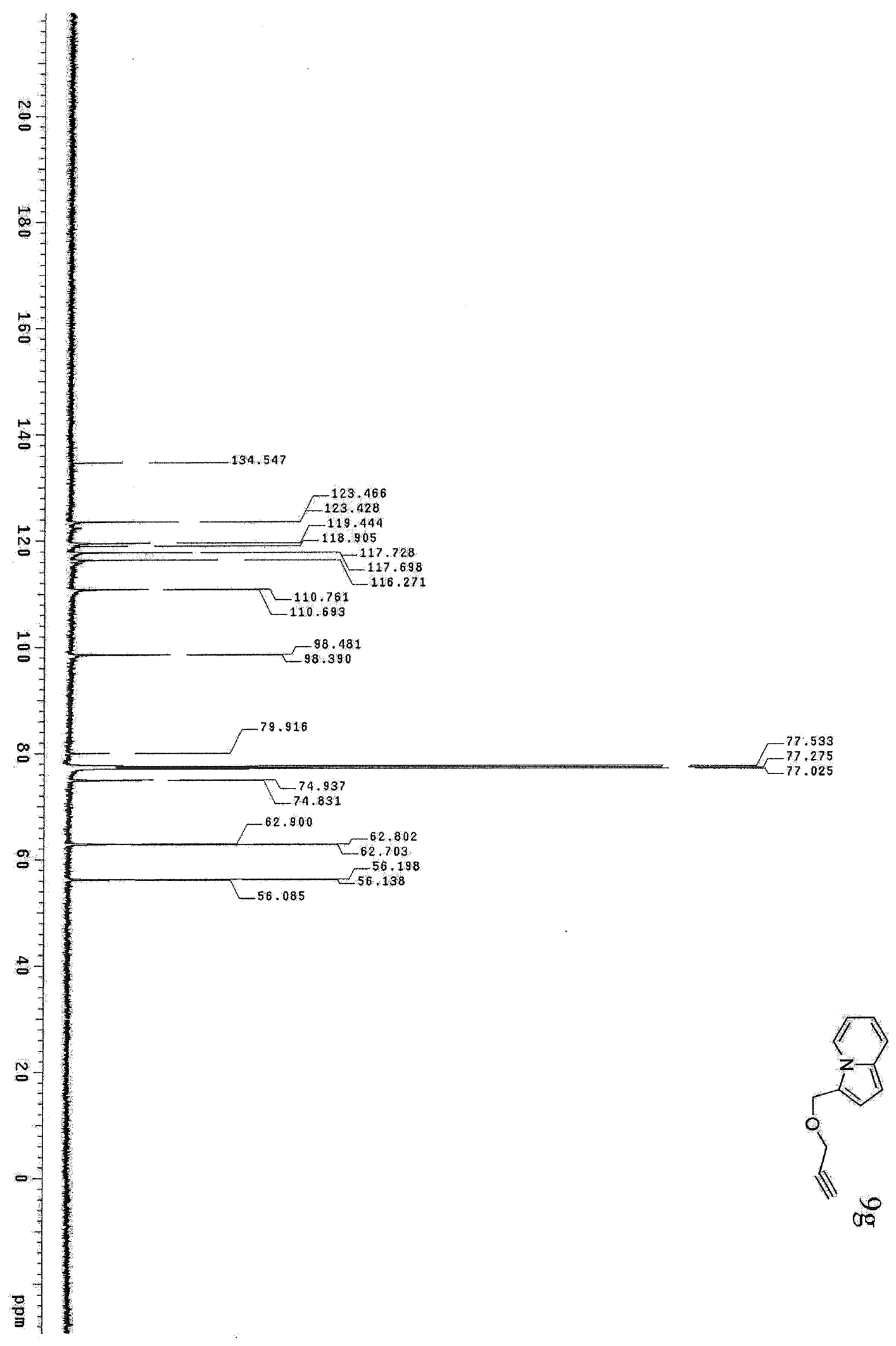



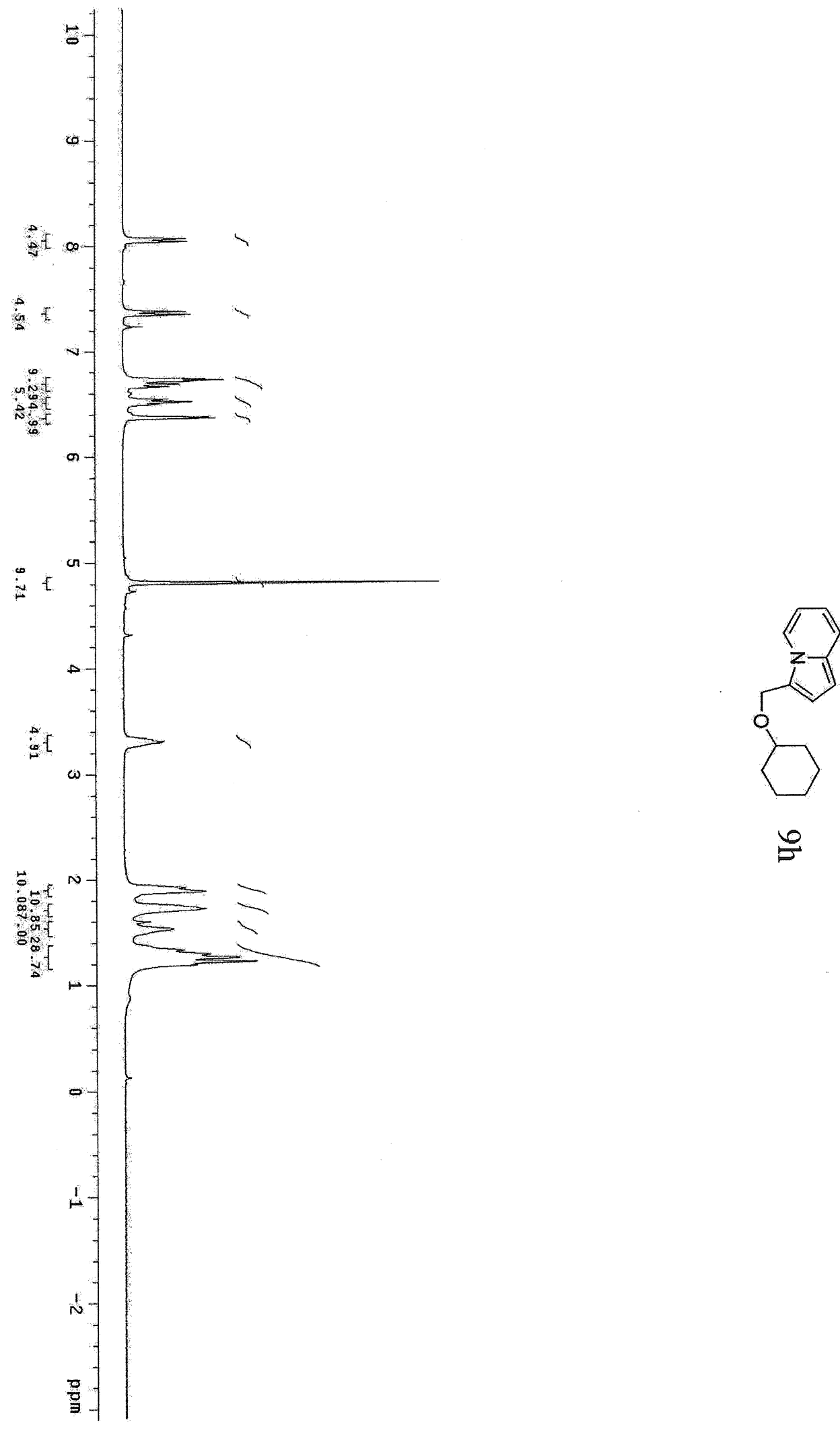
SI 41
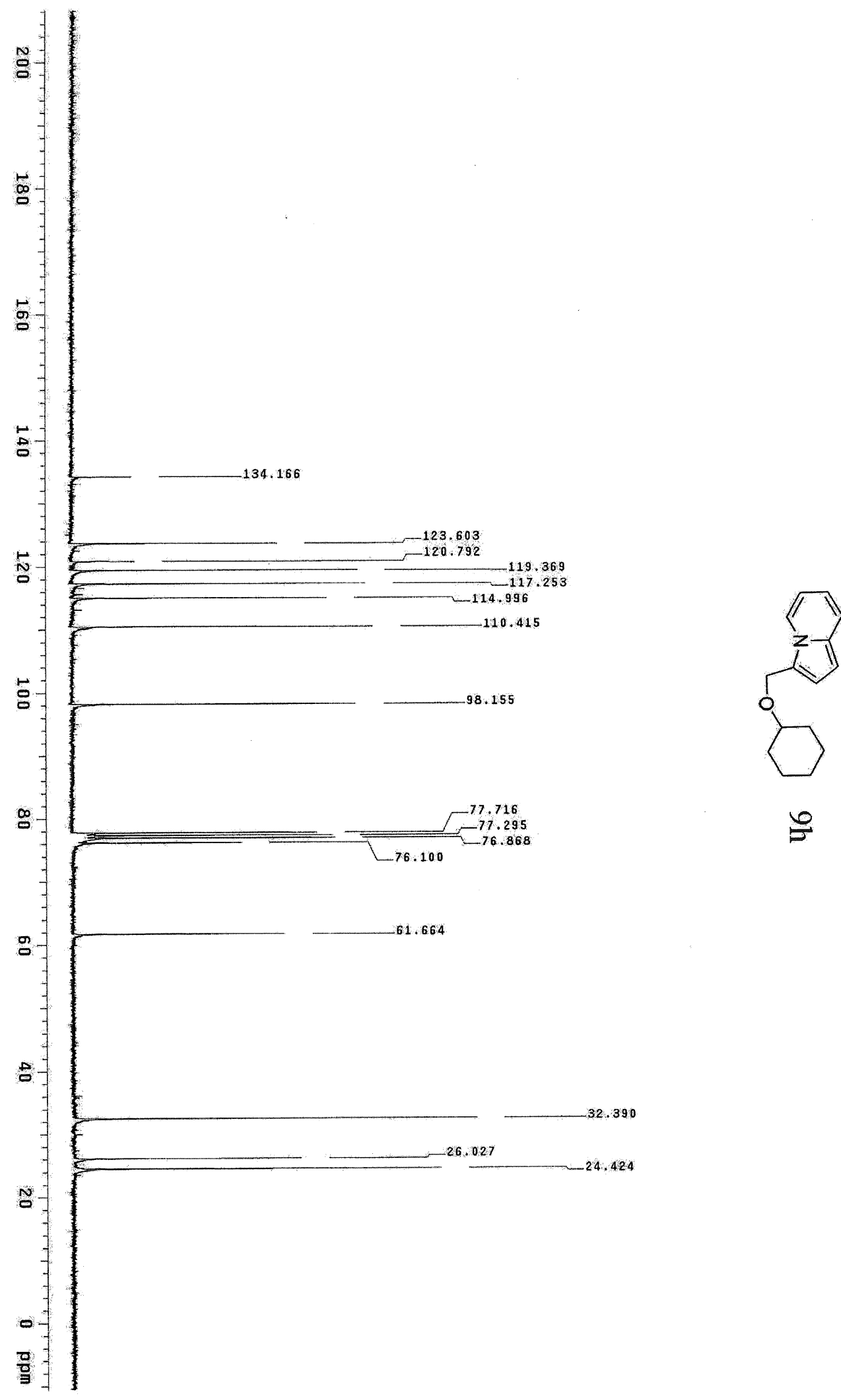
SI 42
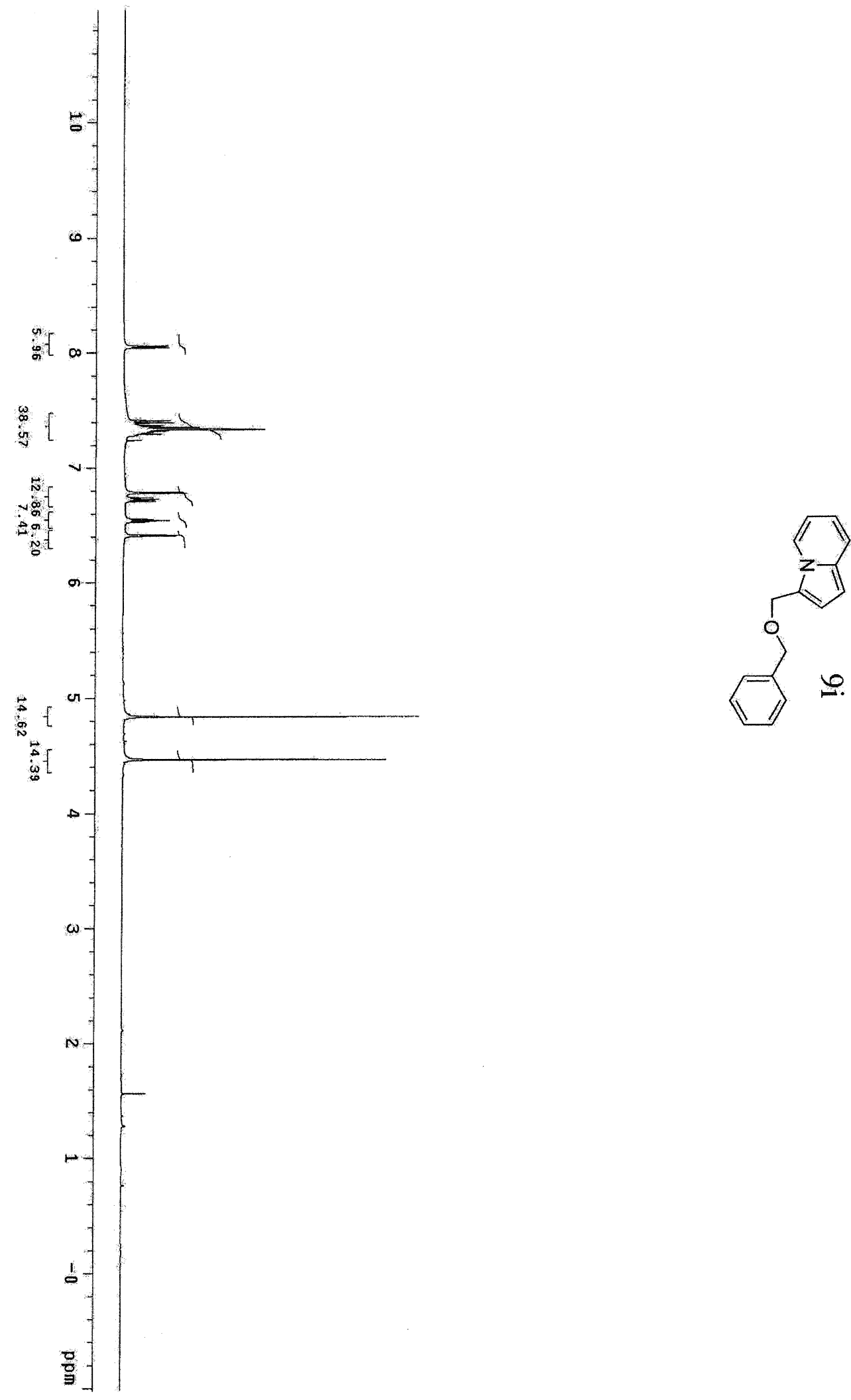

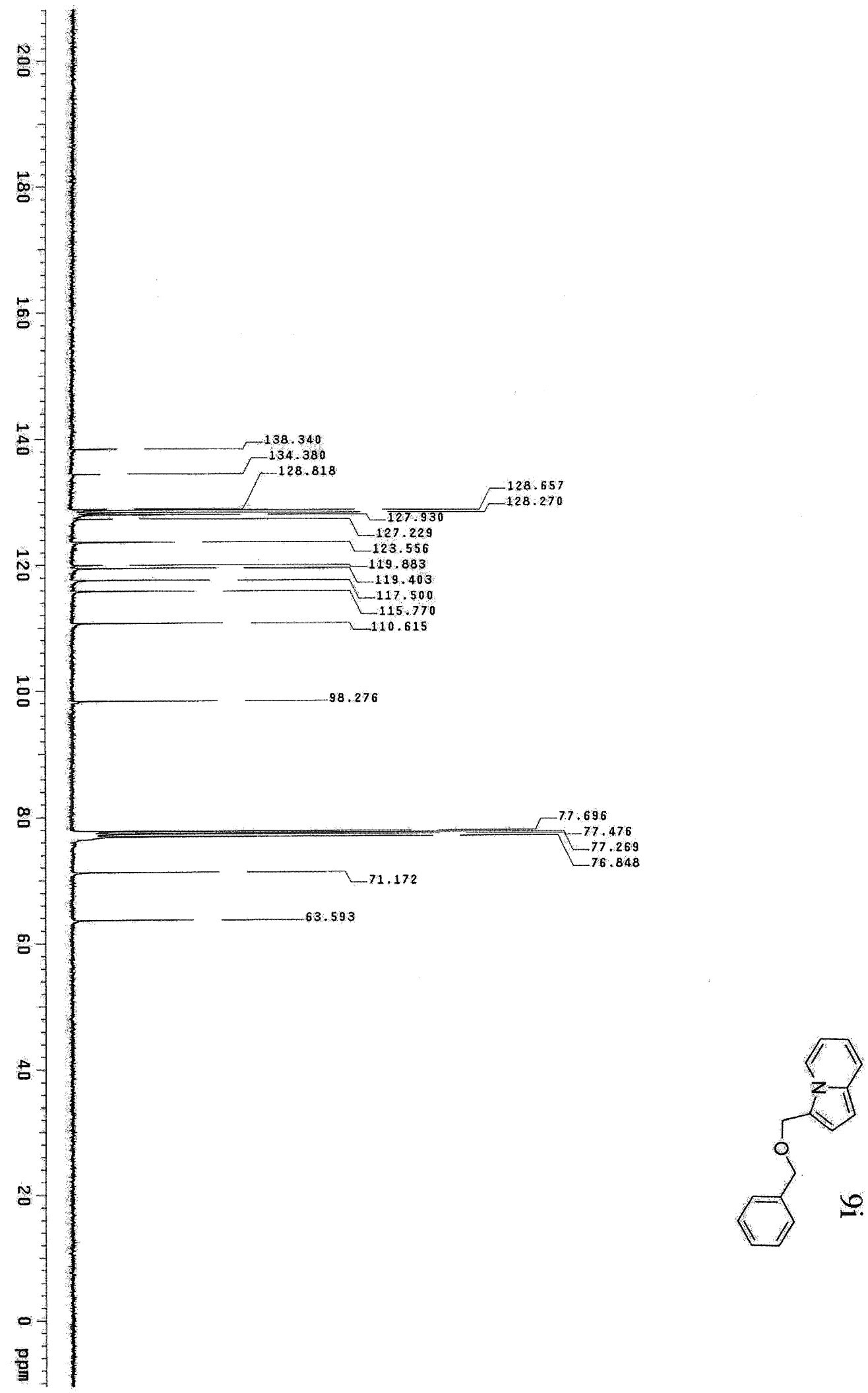

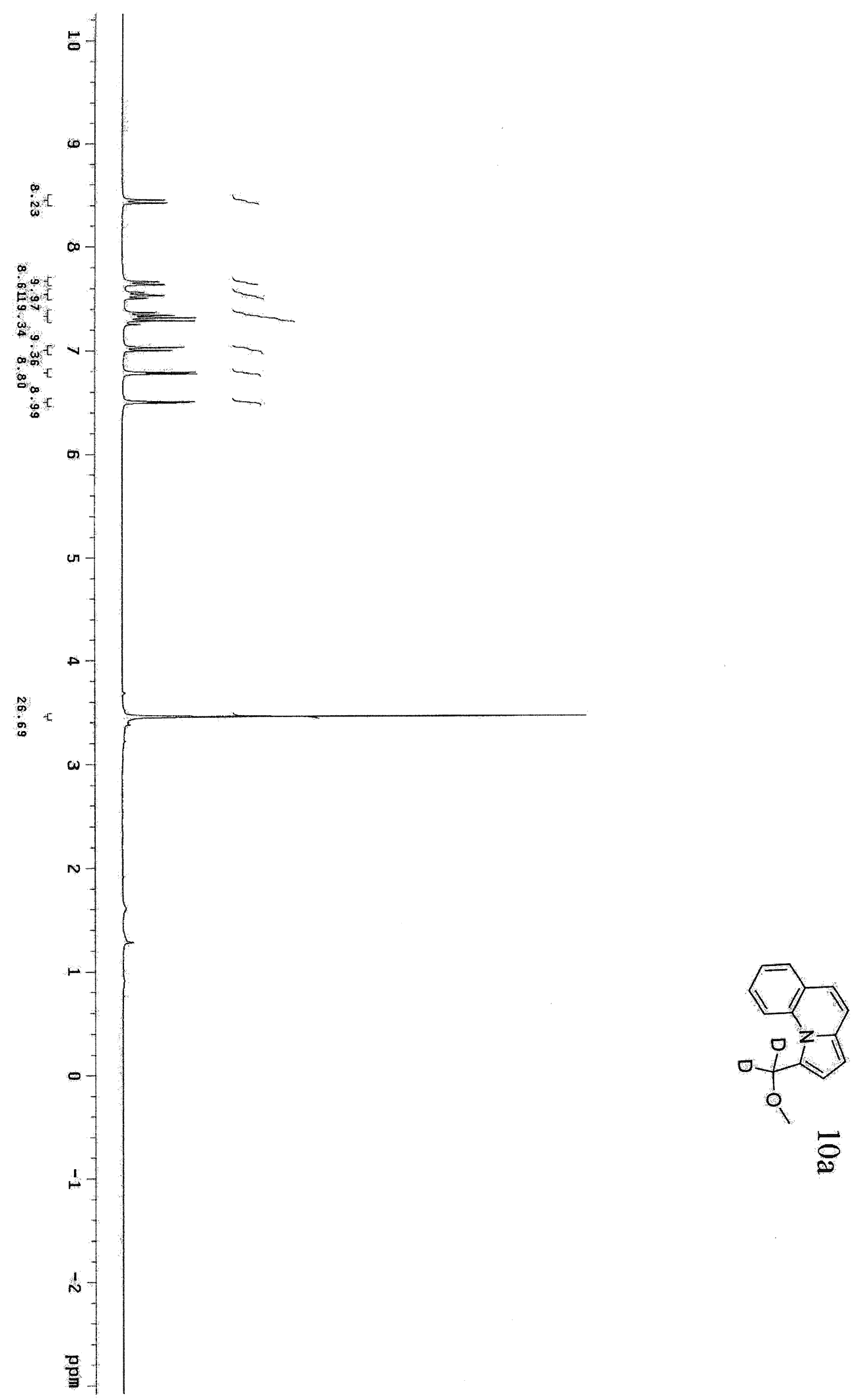


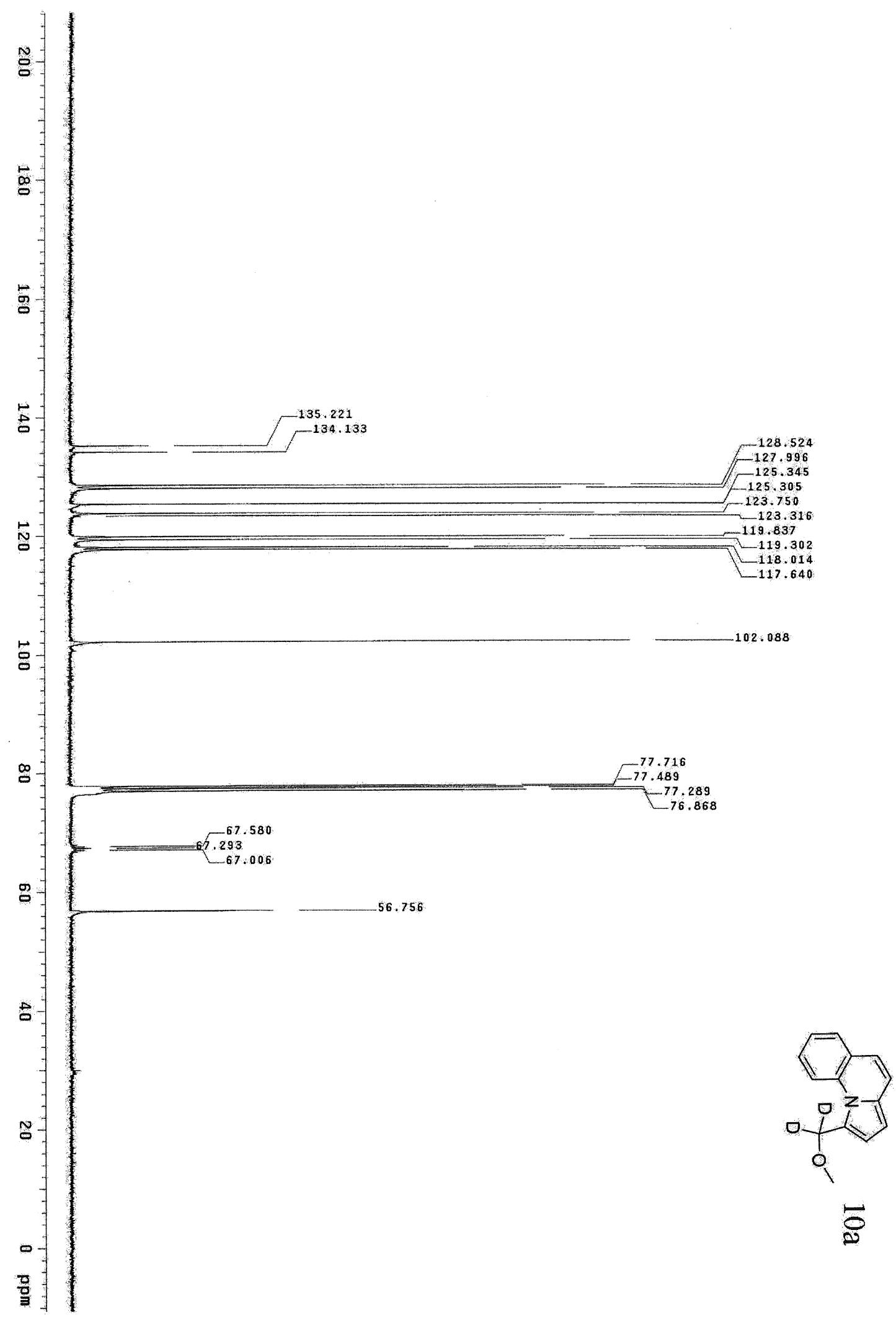



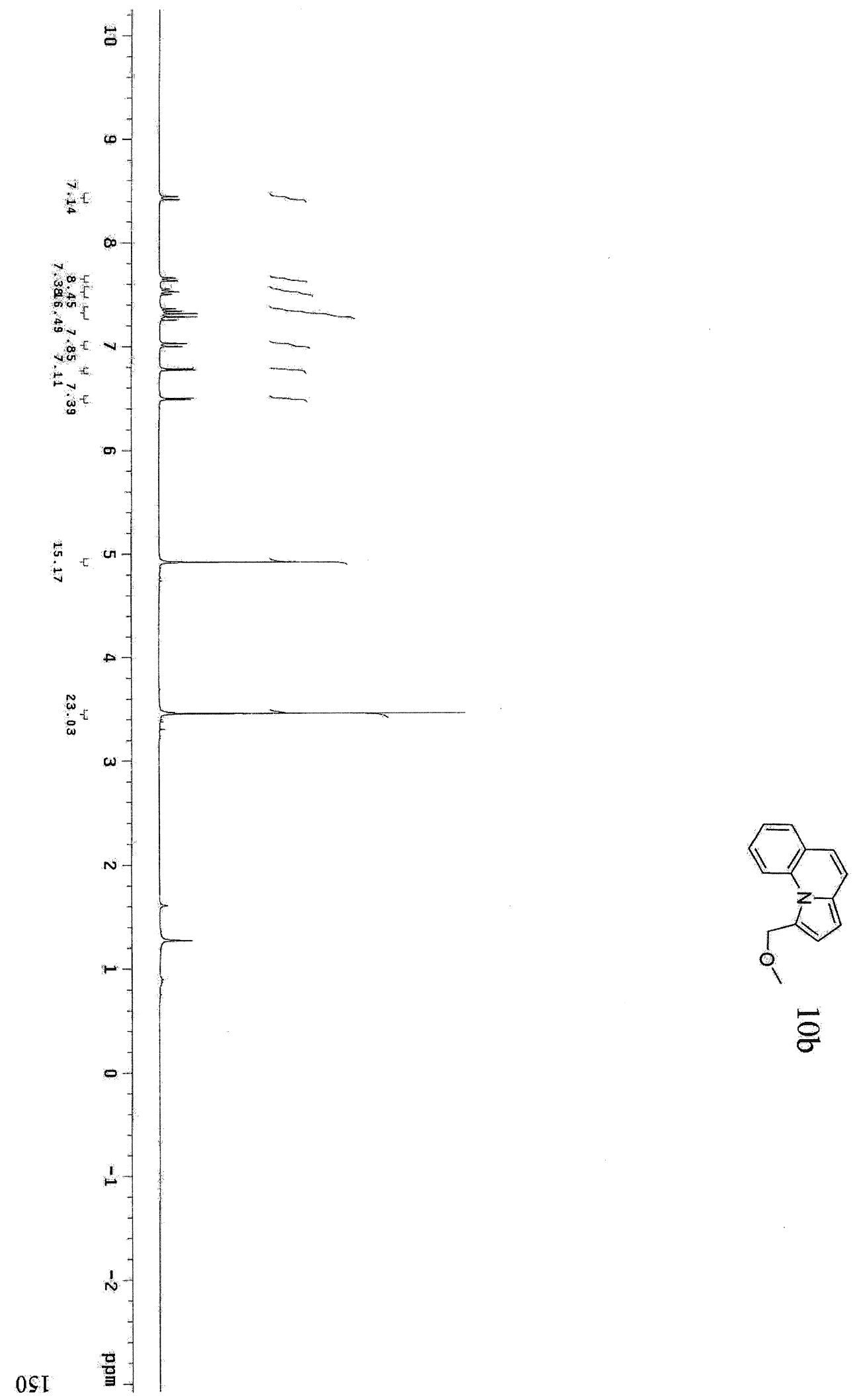


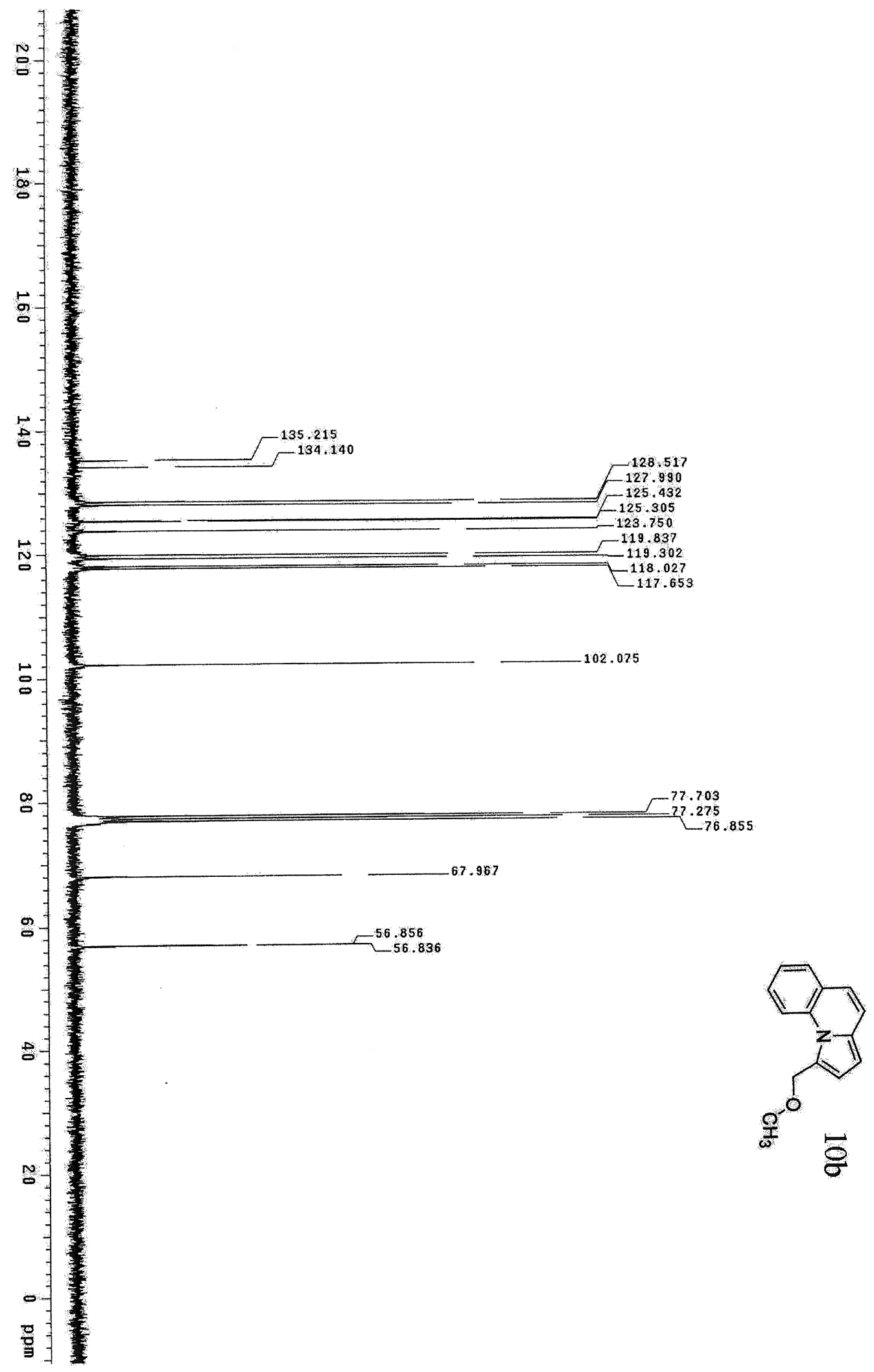



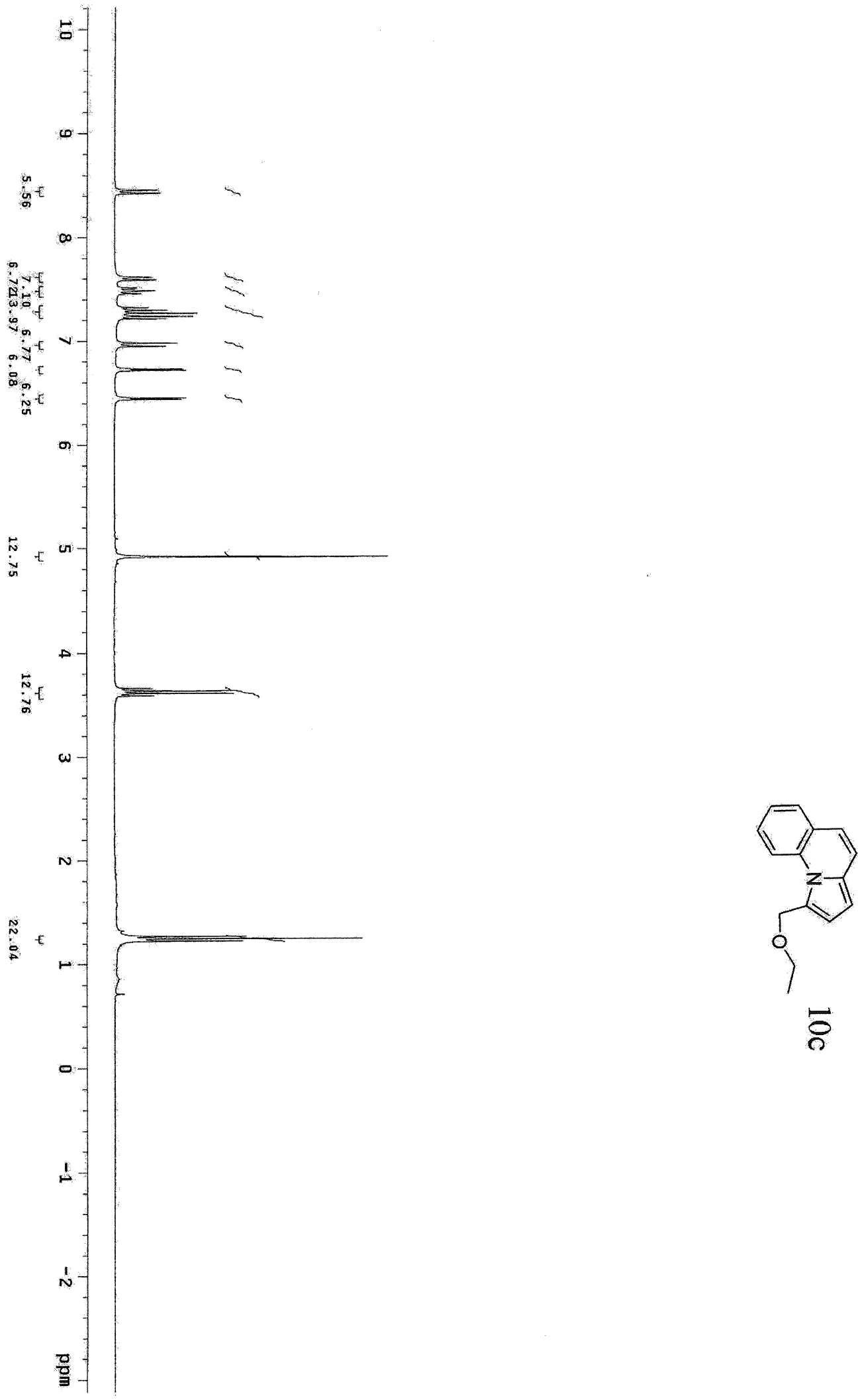

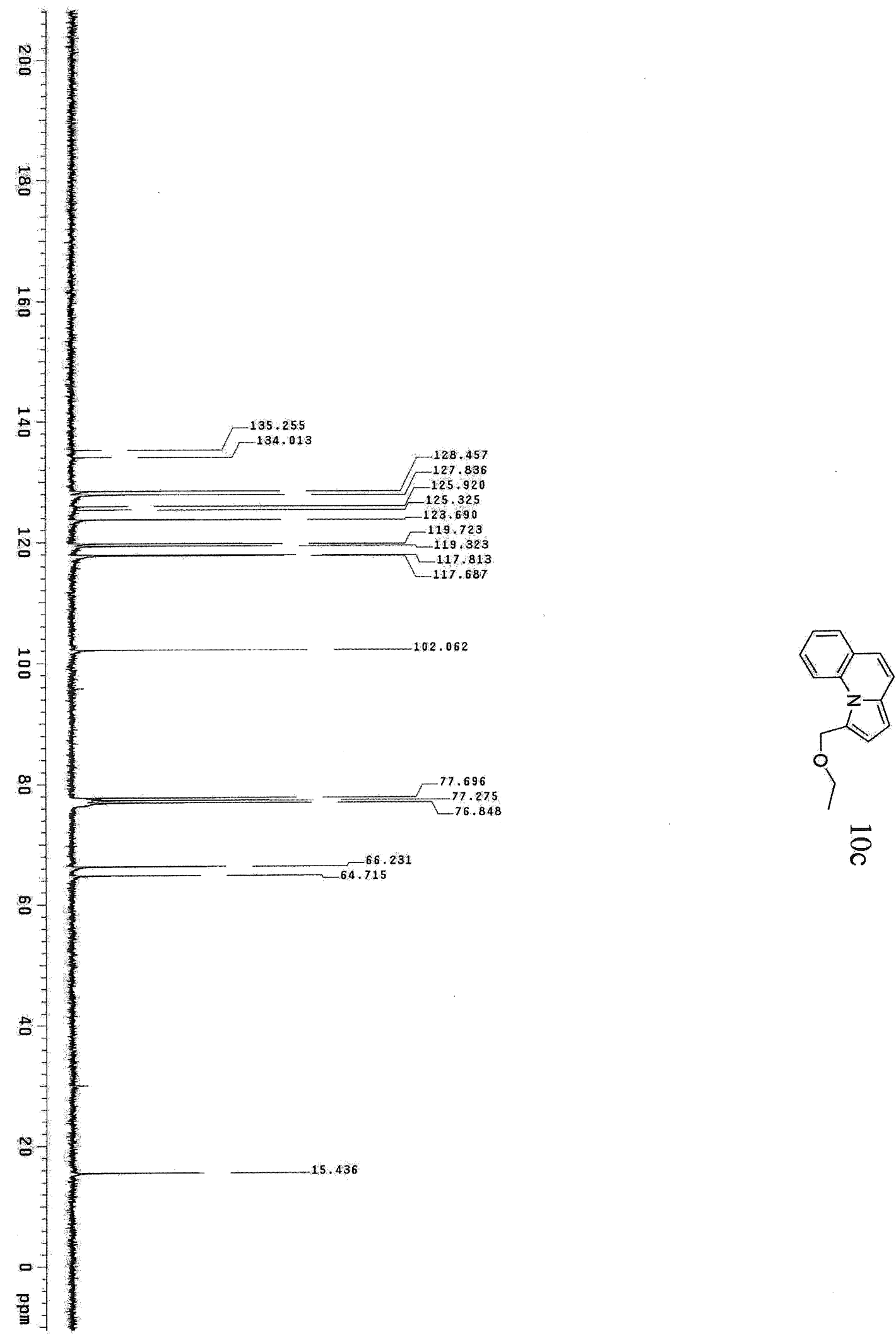

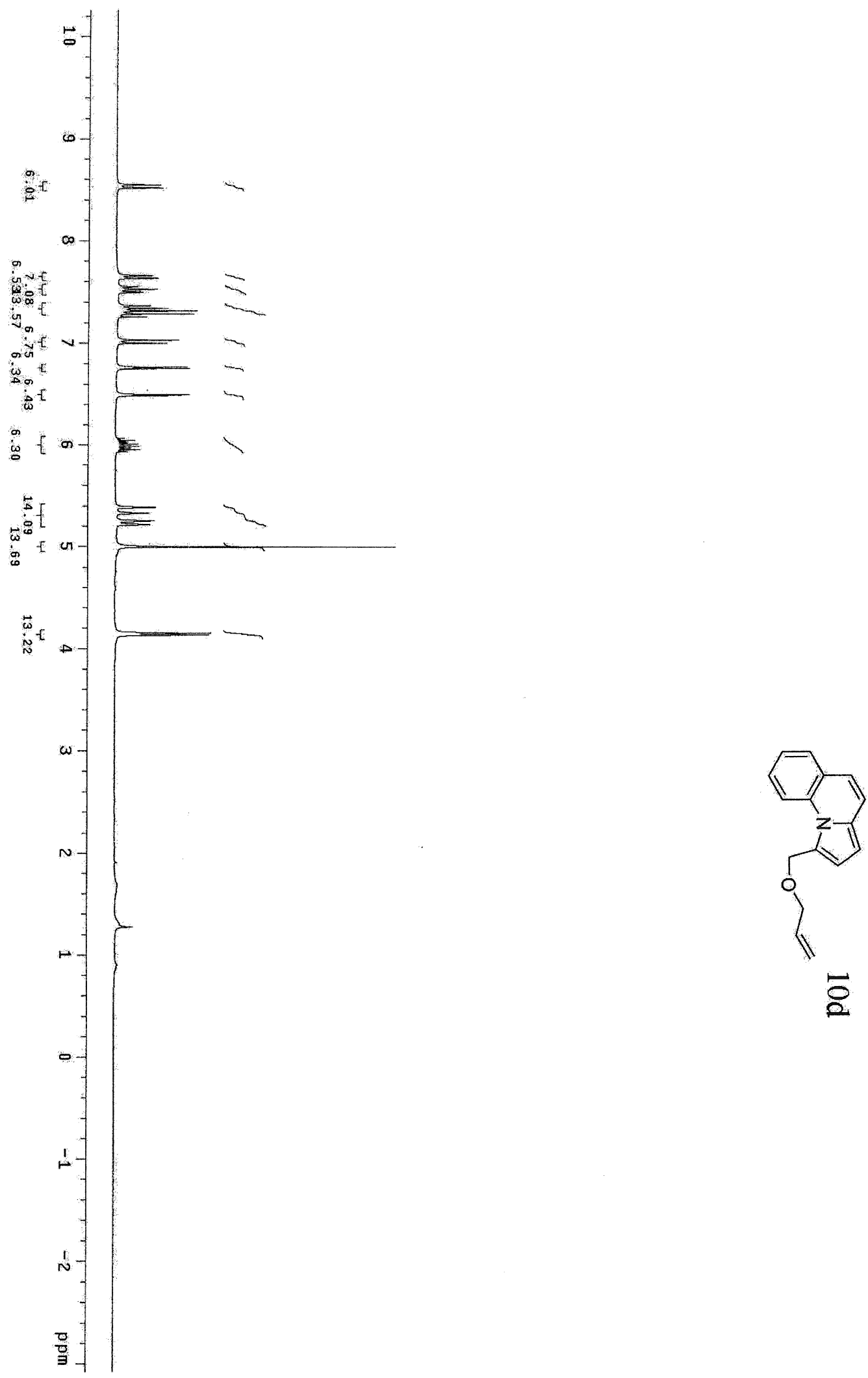

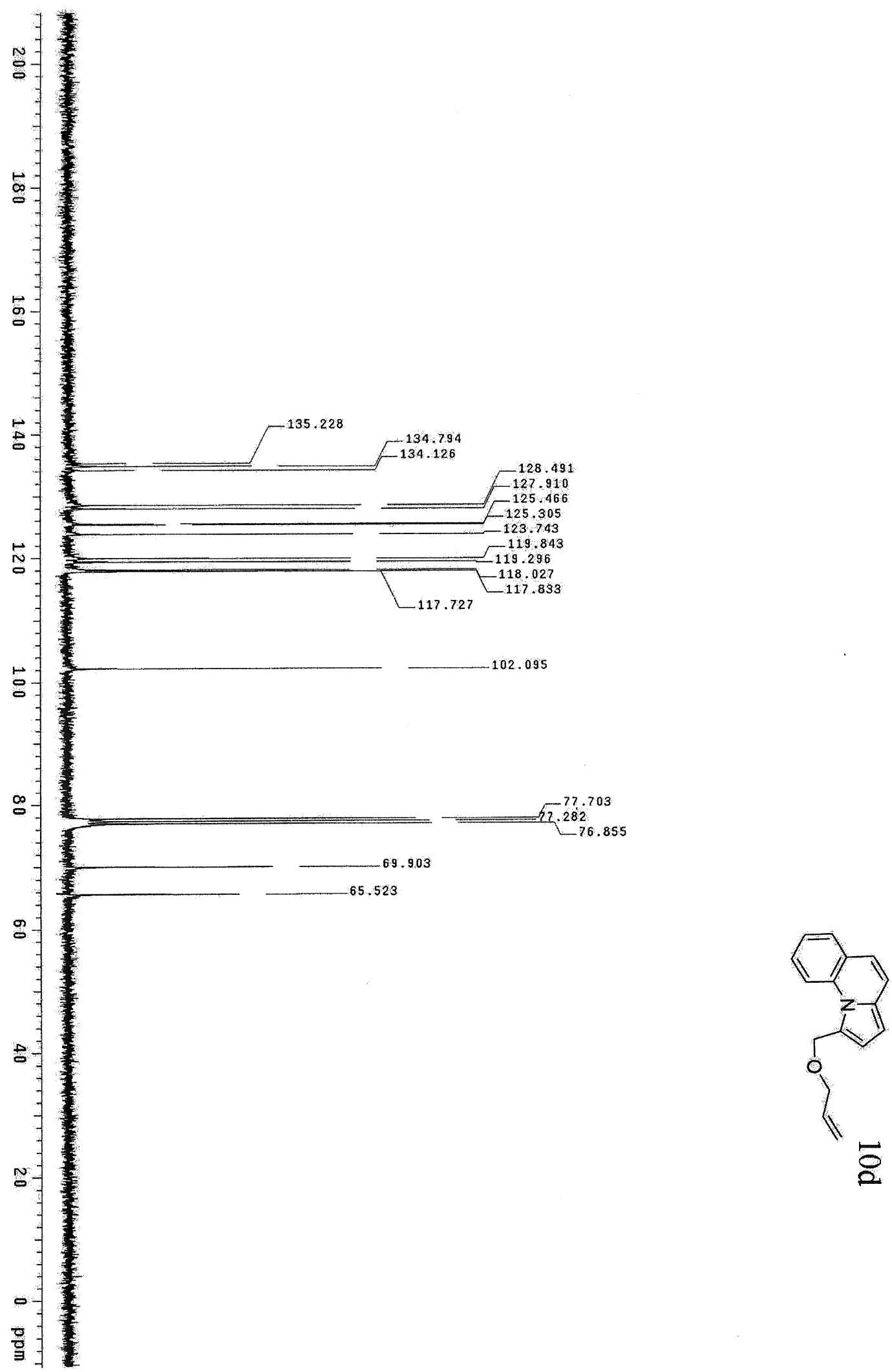

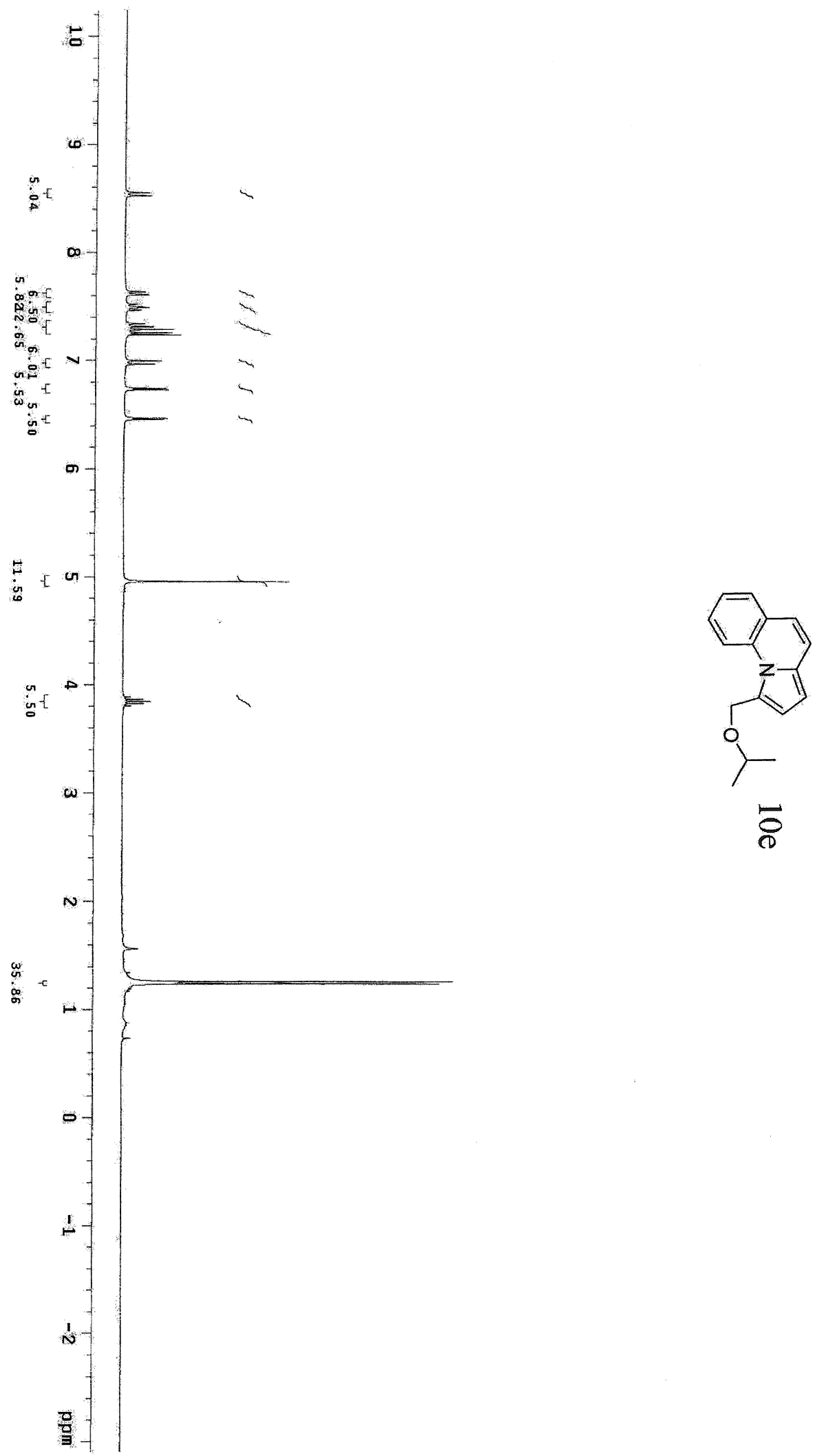

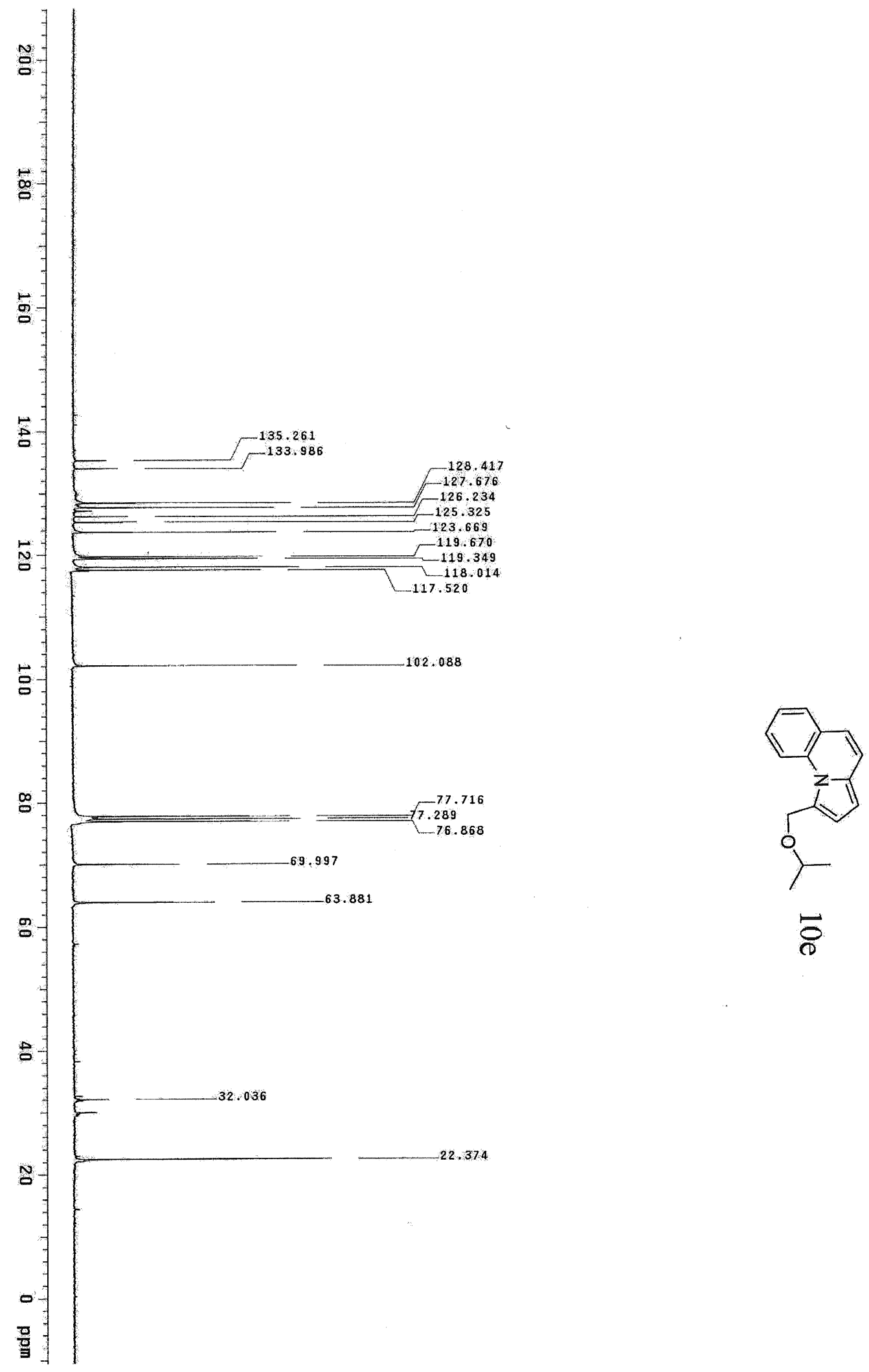


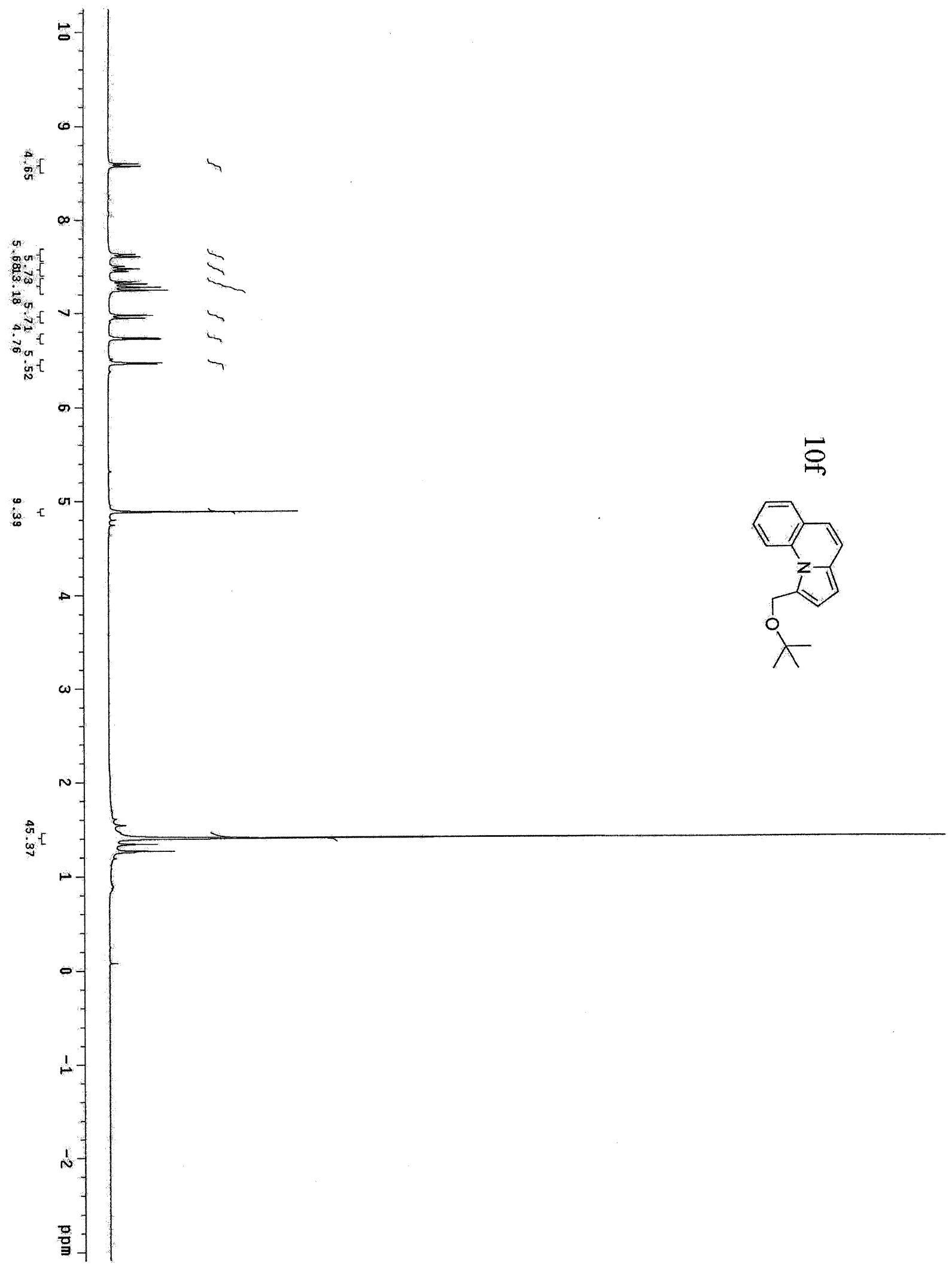



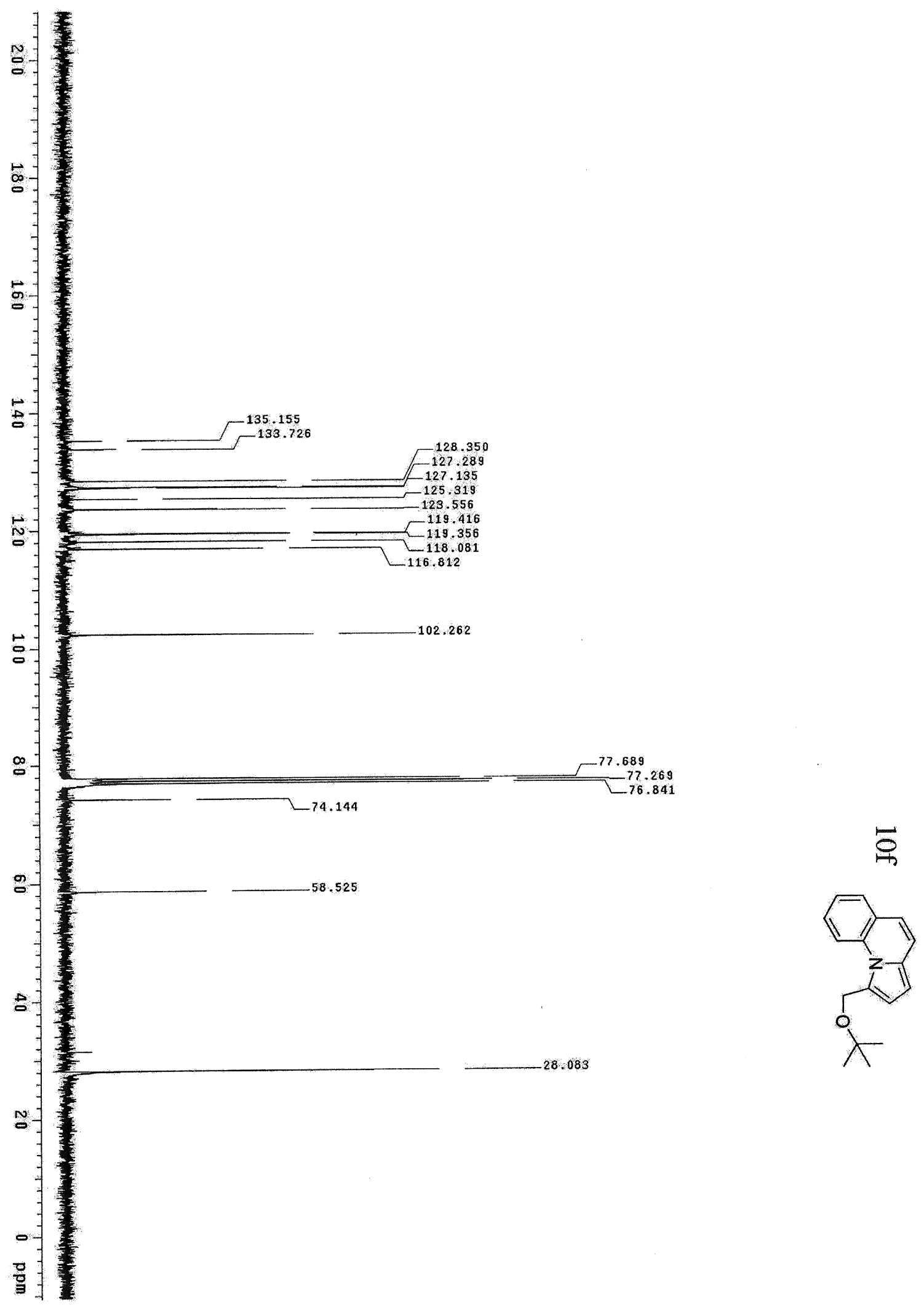

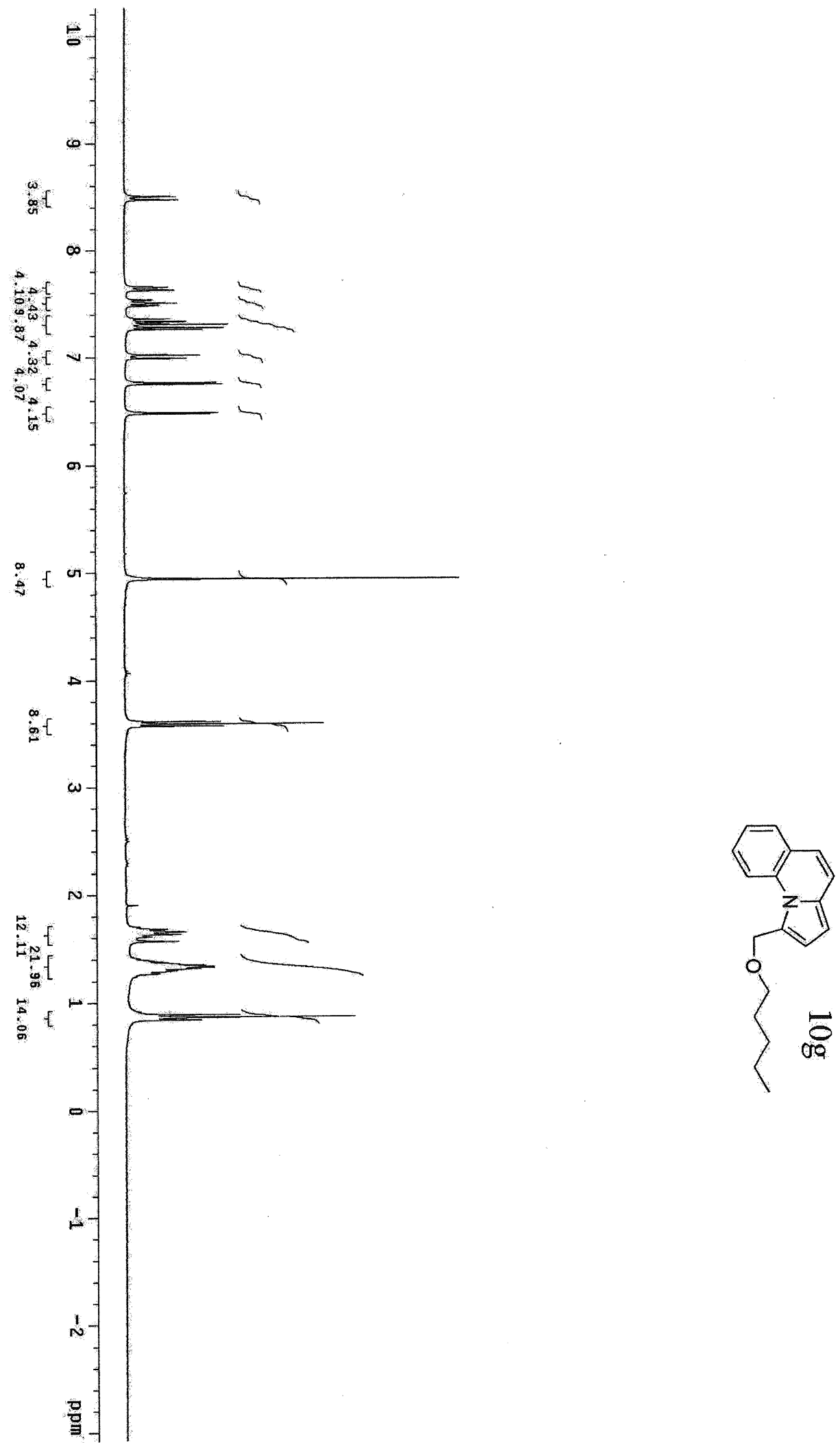

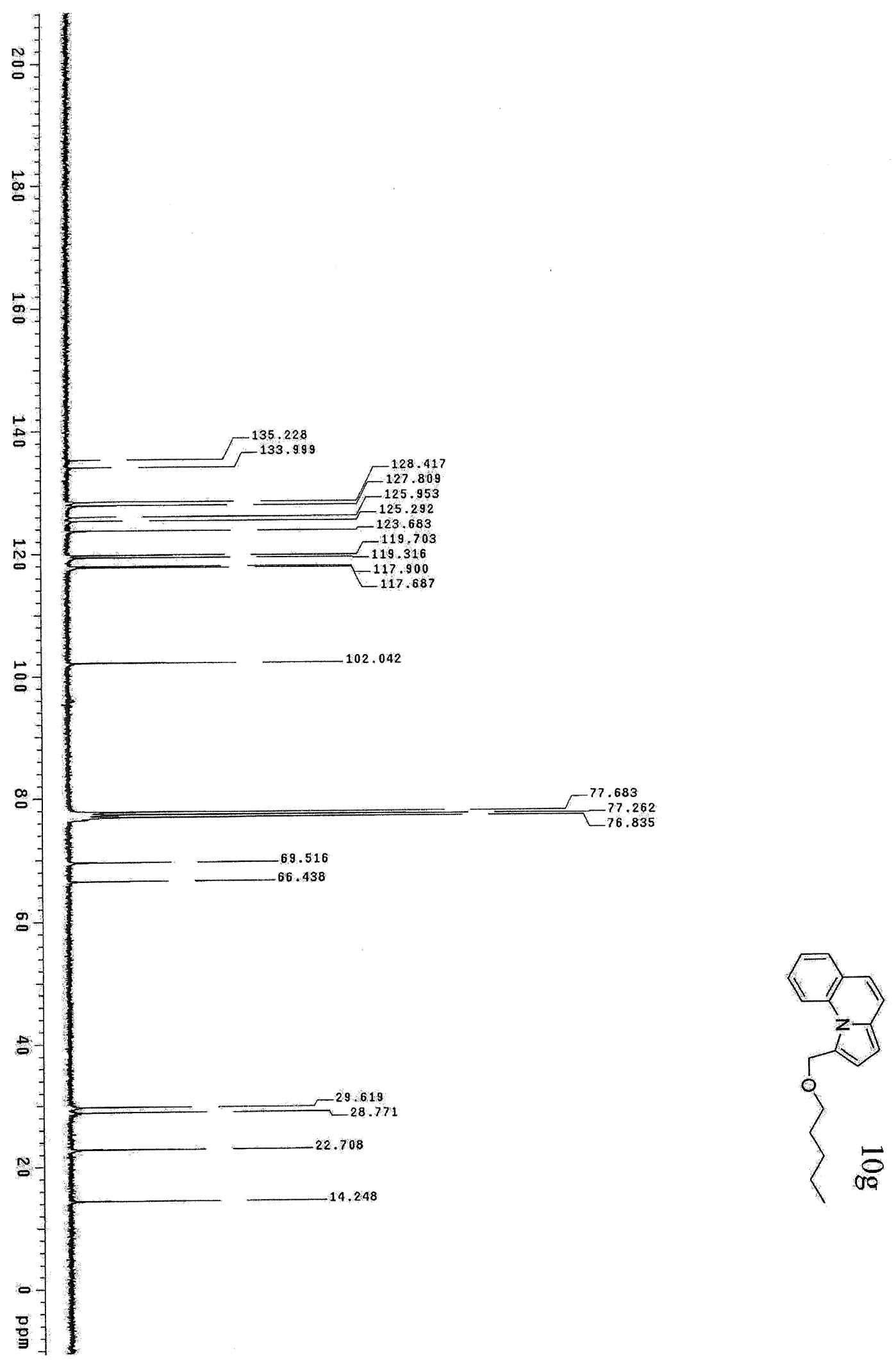

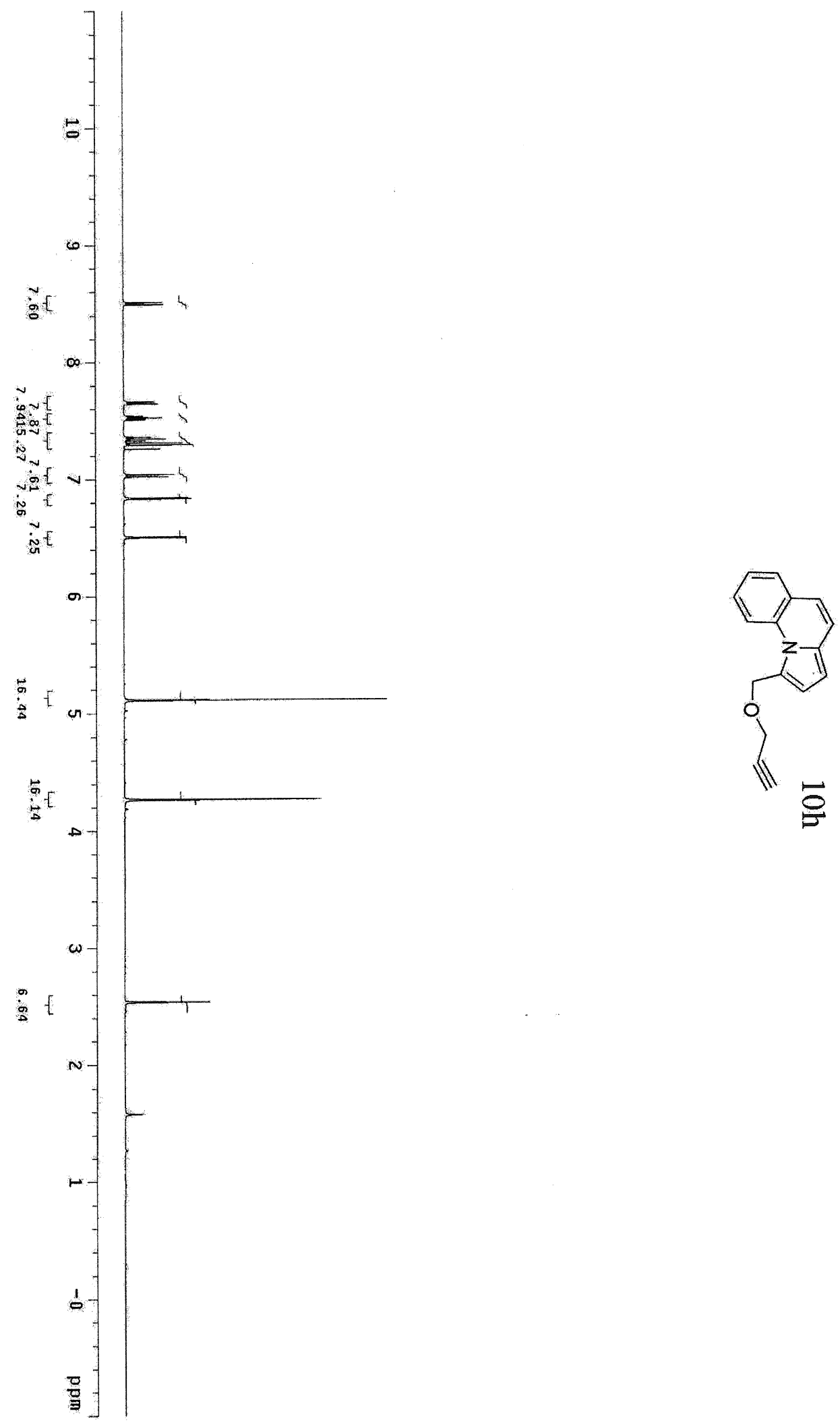

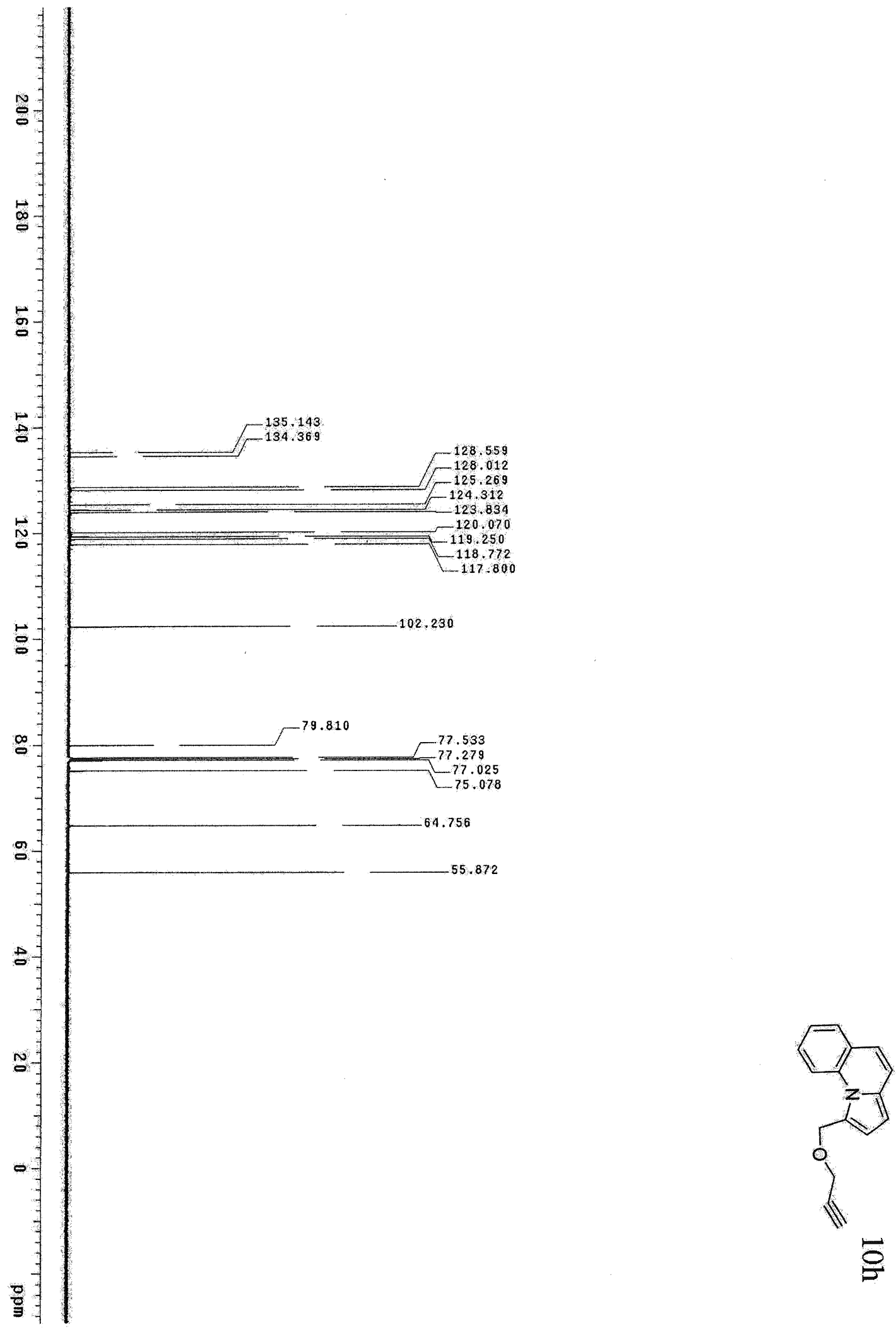

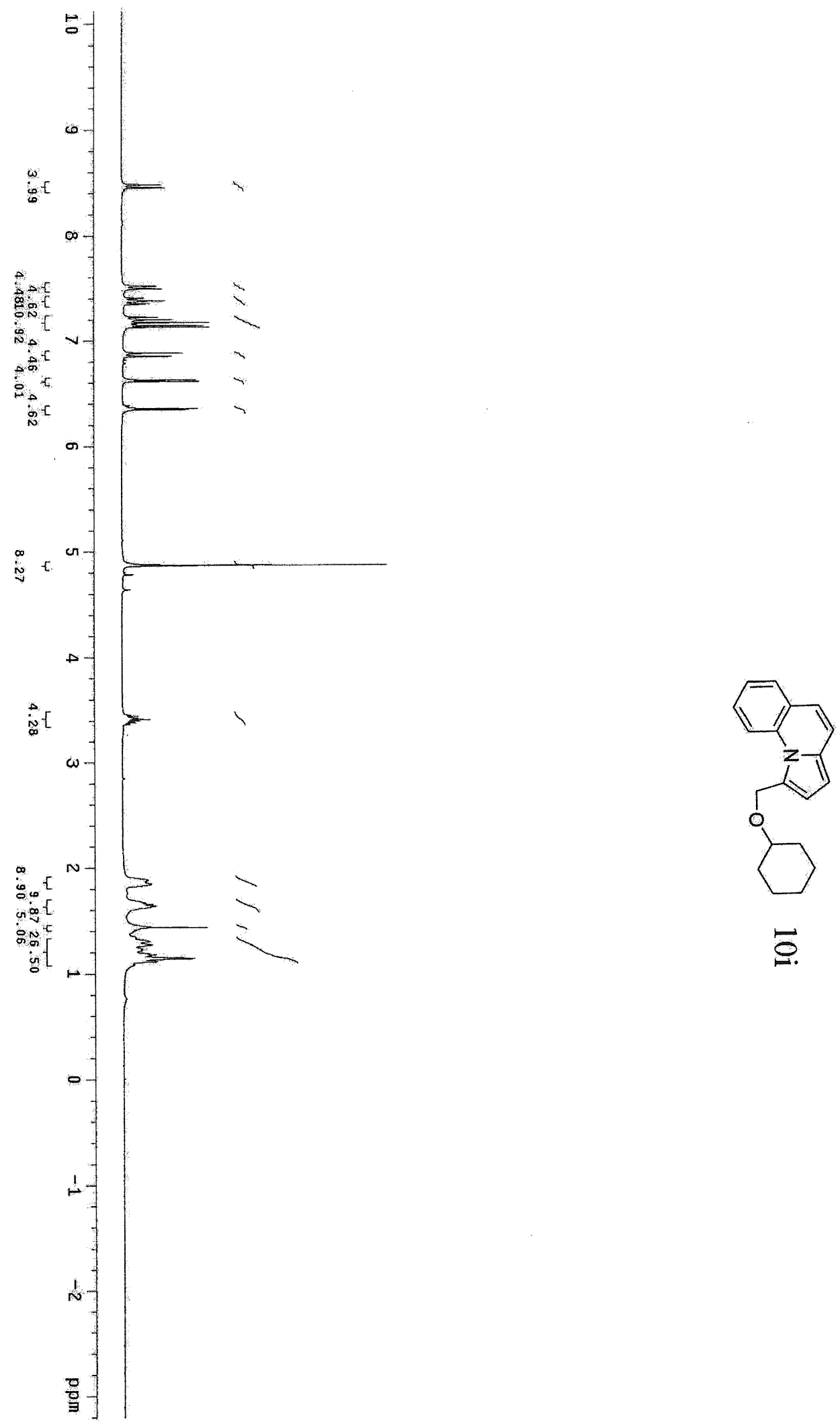
SI 61
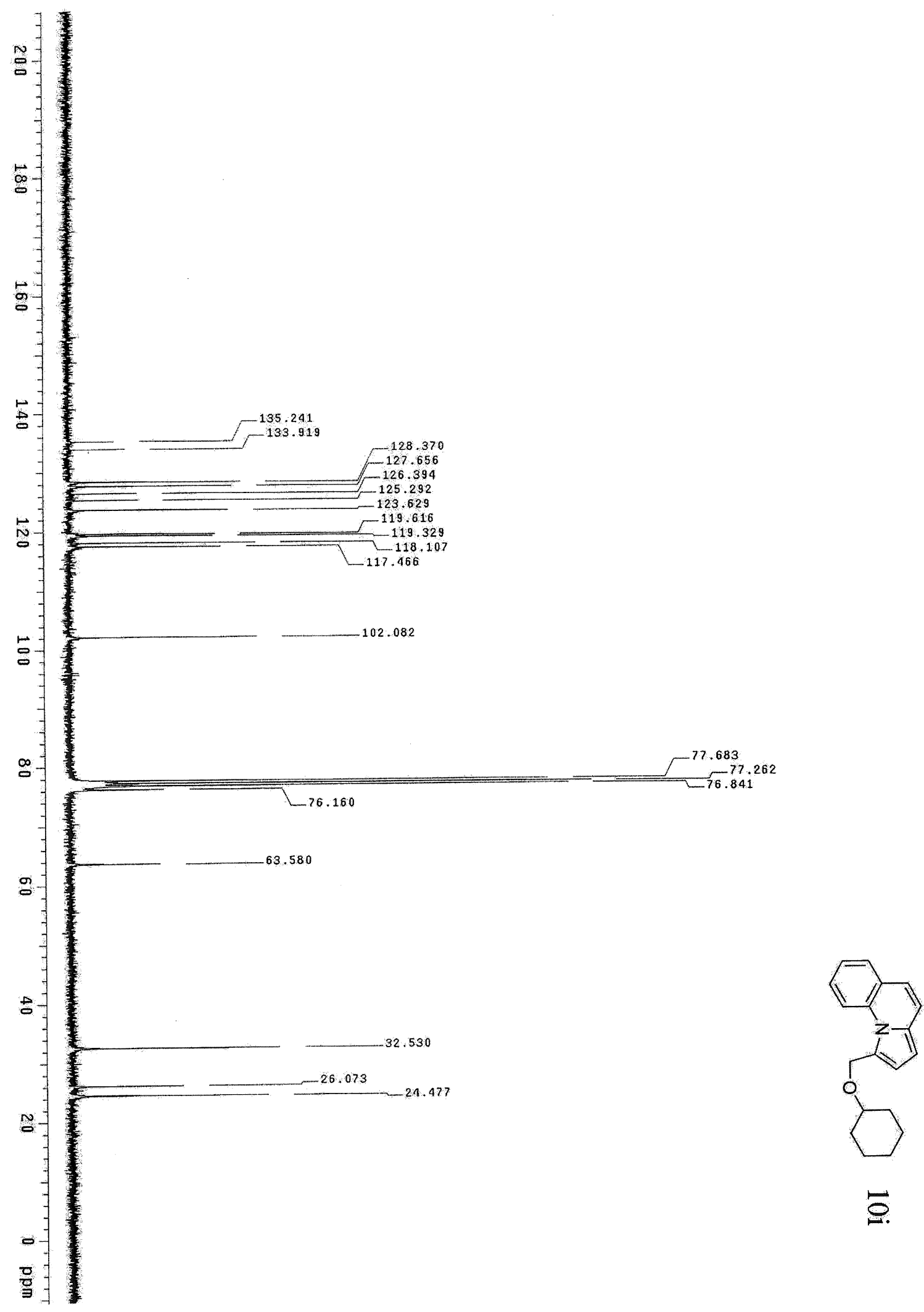

호. 
SI 62
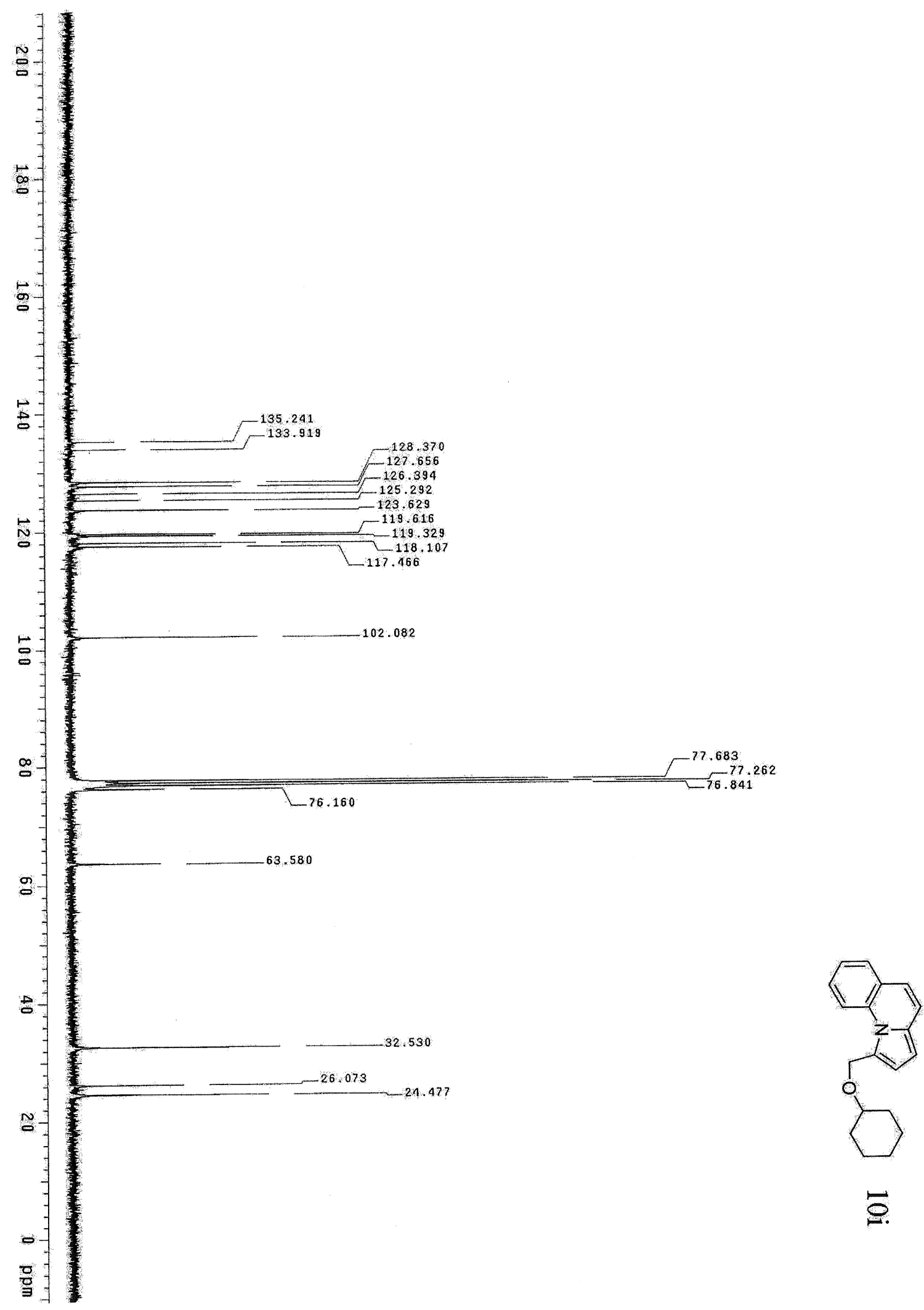

호. 

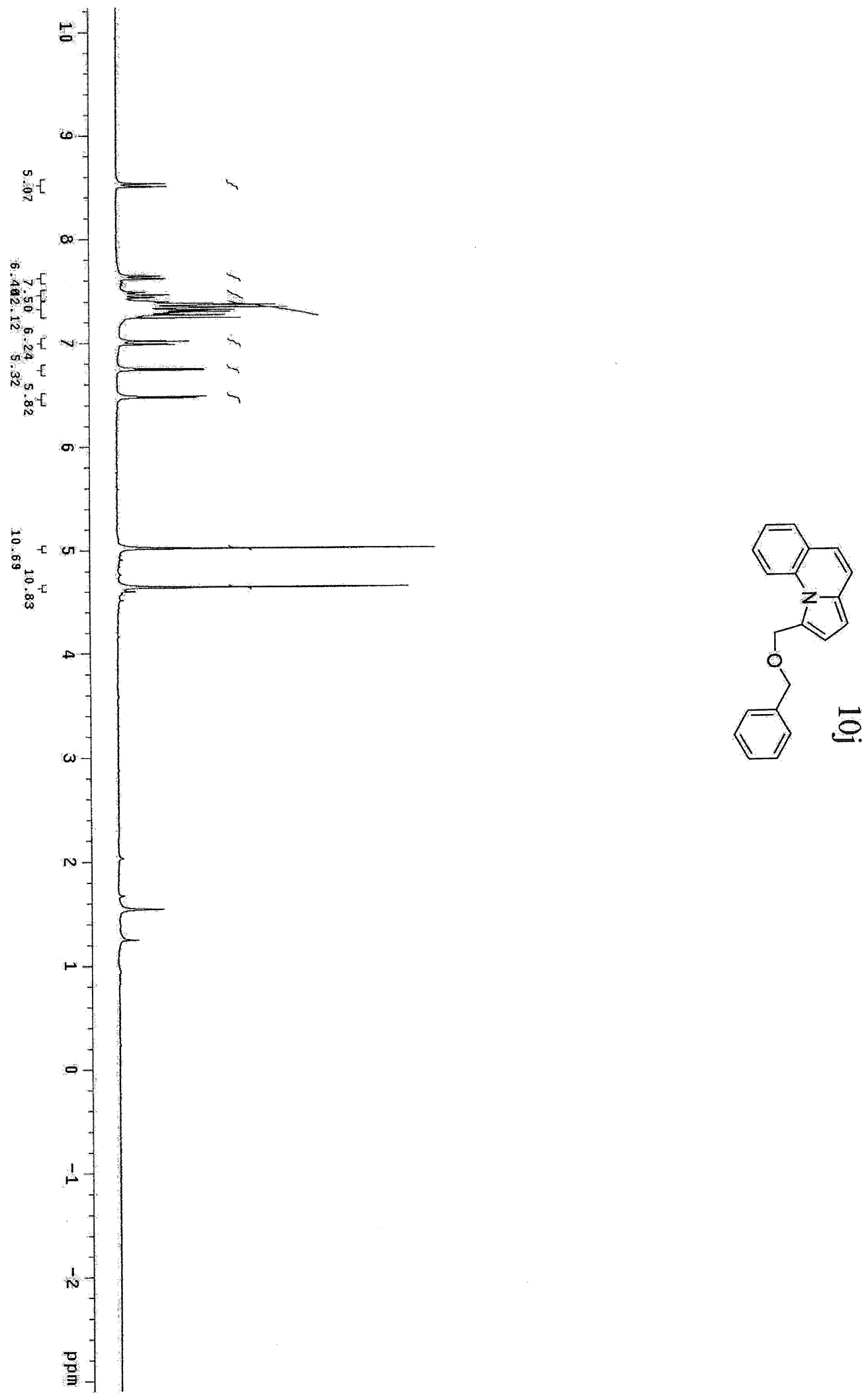

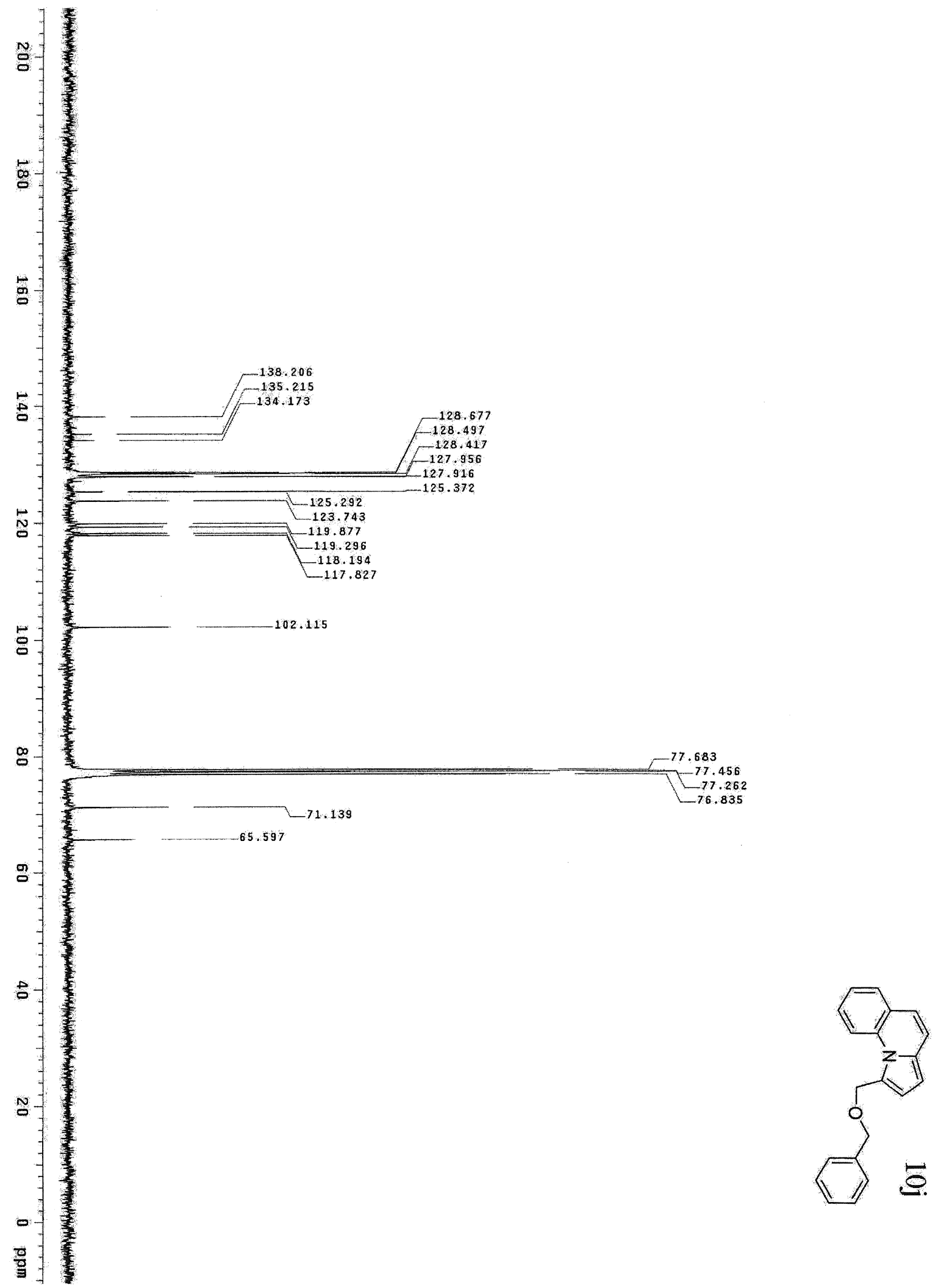